JOURNAL OF THE

AMERICAN MATHEMATICAL SOCIETY

Volume 25, Number 1, January 2012, Pages 189-243

S 0894-0347(2011)00708-X

Article electronically published on June 15, 2011

\title{
STABLE GROUP THEORY AND APPROXIMATE SUBGROUPS
}

\author{
EHUD HRUSHOVSKI
}

\section{INTRODUCTION}

Stable group theory, as developed in the 1970s and 1980s, was an effective bridge between definable sets and objects of more geometric categories. One of the reasons was a body of results showing that groups can be recognized from their traces in softer categories. The first and simplest example is Zilber's stabilizer. Working with an integer-valued dimension theory on the definable subsets of a group $G$, Zilber considered the dimension-theoretic stabilizer of a definable set $X$ : this is the group $S$ of elements $g \in G$ with $g X \triangle X$ of smaller dimension than $X$. Let $X X$ be the product set $X X=\{x y: x, y \in X\}$. If $X$ differs little from $X X$ in the sense that $\operatorname{dim}(X X \triangle X)<\operatorname{dim}(X)$, Zilber showed that $X$ differs little from a coset of $S$.

In the 1990s, Zilber's theory was generalized to the "simple theories" of [49], again initially in a definable finite-dimensional context ([8], 23]). Here the definable sets $X_{t}$ in a definable family $\left(X_{t}: t \in T\right)$ are viewed as "differing little from each other" if simply the pairwise intersections $X_{t} \cap X_{t^{\prime}}$ have the same dimension as each $X_{t}$. Nevertheless it is shown that when the family of translates $(X a: a \in$ $X)$ satisfies this condition, there is a group $H$ of the same dimension as $X$ and with a large intersection with some translate of $X$; this group was still, somewhat inappropriately, called the stabilizer, and we will keep this terminology.

In the present paper we prove the stabilizer theorem in a general first-order setting. A definition is given of being a "near-subgroup" (Definition 3.9), generalizing the stable and simple cases. We then prove the existence of a nearby group (Theorem 3.5$)$. In outline, the proof remains the same as in 23 ; the definability condition on the dimension was removed in 31 . The key is a general amalgamation statement for definable ternary relations, dubbed the "Independence Theorem" (see [8, p. 9 and p. 185). Roughly speaking, in maximal dimension, consistent relations among each pair of types determine consistent relations on a triple; see Theorem 2.22.

The stabilizer obtained in Theorem 3.5 is not a definable group but an $\Lambda$ definable one; it is defined by a countable set of formulas in a saturated model, or alternatively as a group object in the category of projective limits of definable sets. In the finite-dimensional setting of [23], the construction of the stabilizer was complemented by a proof that $\bigwedge$-definable groups are limits of definable groups. This last step is not true at the level of generality considered here: the group of infinitesimals of a Lie group provide counterexamples. We show however that all counterexamples are closely associated with Lie groups; see Theorem 4.2. The proof

Received by the editors August 24, 2010 and, in revised form, May 16, 2011.

2010 Mathematics Subject Classification. 11P70, 03C45.

Research supported in part by Israel Science Foundation grant 1048/07.

(C)2011 American Mathematical Society Reverts to public domain 28 years from publication 
uses the structure theory of Gleason, Montgomery-Zippin and Yamabe for locally compact groups.

A very interesting dictionary between this part of model theory, and certain parts of finite combinatorics, can be obtained by making the model-theoretic "dimension $n$ " correspond to the combinatorial "cardinality of order $c^{n}$ " (cf. 8, 8.4). Nearsubgroups in the above sense then correspond to asymptotic families of finite subsets $X$ of a group (or a family of groups), with $\left(X \cup X^{-1}\right)^{3} /|X|$ bounded. Equivalently (see [50, Lemma 3.4, and Corollary 3.11 below) $\left|X^{k}\right| /|X|$ is bounded for any given $k$. Subsets of groups with weak closure conditions were considered in combinatorics at least since 14. An excellent survey centering on rings can be found in the first pages of [51; see also [50] for more general noncommutative groups. The parallels to the model-theoretic development are striking. We turn now to a description of some consequences of the stabilizer theorem in this combinatorial setting.

For the sake of the introduction we consider finite subsets of $G$ (more general situations will be allowed later.) We recall Terence Tao's notion of an approximate subgroup. A finite subset $X \subseteq G$ is said to be a $k$-approximate group if $1 \in X, X=$ $X^{-1}$, and $X X$ is contained in $k$ right cosets of $X$. Say $X, Y$ are commensurable if each is contained in finitely many right cosets of the other, with the number bounded in terms of $k$. It is felt that approximate subgroups should be commensurable to actual subgroups, except in situations involving Abelian groups in some way. See 52 for a compelling exposition of the issue.

Gromov's theorem [18 on finitely generated groups of polynomial growth fits into this framework, taking $X$ to be a ball of size $2^{n}$ in the Cayley graph, for large $n$; then $X$ is a $2^{d}$-approximate subgroup, where $d$ is the growth exponent. Gromov shows that the group is nilpotent, up to finite index.

Theorem 4.2 says nothing about a fixed finite approximate subgroup, but it does have asymptotic consequences to the family of all $k$-approximate subgroups for fixed $k$. In particular, we obtain:

Theorem 1.1. Let $f: \mathbb{N}^{2} \rightarrow \mathbb{N}$ be any function, and fix $k \in \mathbb{N}$. Then there exist $e^{*}, c^{*}, N \in \mathbb{N}$ such that the following holds.

Let $G$ be any group, $X$ a finite subset, and assume $\left|X X^{-1} X\right| \leq k|X|$.

Then there are $e \leq e^{*}, c \leq c^{*}$, and subsets $X_{N} \subseteq X_{N-1} \subseteq \cdots \subseteq X_{1} \subseteq$ $X^{-1} X X^{-1} X$ such that $X, X_{1}$ are e-commensurable, and for $1 \leq m, n<N$ we have:

(1) $X_{n}=X_{n}^{-1}$.

(2) $X_{n+1} X_{n+1} \subseteq X_{n}$.

(3) $X_{n}$ is contained in the union of c translates of $X_{n+1}$.

(4) $\left[X_{n}, X_{m}\right] \subseteq X_{k}$ whenever $k \leq N$ and $k<n+m$.

(5) $N>f(e, c)$.

Roughly speaking, this is deduced as a special case of the following principle: if a sentence of a certain logic holds for all compact neighborhoods of the identity in all finite-dimensional Lie groups, then it holds for all approximate subgroups. We have not explicitly determined the relevant logic; Proposition 6.6 hints that, given further work on the first-order theory of Lie groups with distinguished closed subsets, much stronger transfer principles may be possible than what we have used.

The first three clauses of Theorem 1.1 suggest a part of a noncommutative Bourgain system as defined in [17], and conjectured by Ben Green in [52 to exist for approximate subgroups. Green's conjecture was in part intended to show that "one 
can do a kind of approximate representation theory", which can be viewed as a description of Theorem 4.2 and the deduction between the two.

The fourth clause suggests a kind of topological nilpotence. Note that (4) implies that $\left[X_{1}, X_{1}\right] \subseteq X_{1}$. For a set of generators of a finite simple group, this in itself seems to be a curious property.

The use of the structure theory of locally compact groups here follows Gromov 18. But the bridge to locally compact groups is a different one: Gromov's is metric, while ours is measure-theoretic.

It is natural to consider a somewhat more general framework. Call a pair $\left(X, G, \cdot,^{-1}, 1\right)$ a Freiman approximate group if $X$ is a finite subset of $G, \cdot: X^{(2)} \rightarrow G$ and ${ }^{-1}: X \rightarrow X$ are functions, such that for any $\left(x_{1}, \ldots, x_{12}\right) \in X^{12}$, the iterated products $\left(\left(x_{1} \cdot x_{2}\right) \cdot\left(x_{3} \cdot \ldots\right)\right)$ are defined and independent of the placing of the parentheses, $x x^{-1}=x^{-1} x=1 \in X, 1 \cdot x=x \cdot 1=x$, and $\left|X X^{-1} X\right| /|X| \leq k|X|$. Then Theorem 1.1 is also valid for Freiman approximate groups. In particular $X$ has a large subset $X_{2}$ closed under [,] if not under ·, and in fact with $\left[X_{2}, X_{2}\right]^{2} \subseteq X_{2}$. This again suggests that approximateness can only really enter via an Abelian part of a structure. This "local" version uses local versions of the theory of locally compact groups due to Goldbring [16].

The finiteness assumption on $X$ in the above results is really only used via the counting measure "at the top dimension", so they remain valid in a measuretheoretic setting; see Theorem 4.15.

The remaining corollaries of Theorem 3.5 attempt to make a stronger use of finiteness. They are proved directly, without Lie theory, and go in a somewhat complementary direction. The first assumes that the group generated by an approximate subgroup $X$ is perfect in a certain strong statistical sense. The conclusion is that $X$ is close to an actual subgroup. We write $a^{X}=\left\{x^{-1} a x: x \in X\right\}$.

Corollary 1.2. For any $k, l, m \in \mathbb{N}$, for some $p<1, K \in \mathbb{N}$, we have the following statement.

Let $G$ be a group, $X_{0}$ a finite subset, $X=X_{0}^{-1} X_{0}$. Assume $\left|X_{0} X\right| \leq k\left|X_{0}\right|$. Also assume that with probability $\geq p$, an l-tuple $\left(a_{1}, \ldots, a_{l}\right) \in X^{l}$ satisfies: $\left|a_{1}^{X} \cdots a_{l}^{X}\right| \geq$ $|X| / m$.

Then there exists a subgroup $S$ of $G, S \subseteq X^{2}$, such that $X$ is contained in $\leq K$ cosets of $S$.

We could use $\left(a^{X} \cup\left(a^{-1}\right)^{X}\right)^{(l)}$ (or $\left.a^{X_{0}}\right)$ in place of $a^{X}$ above. See Theorem 3.12 for a weaker alternative version of the hypotheses. $p$ can be taken to be a recursive function of $k, l, m$, but I have made no attempt to estimate it. As Ward Henson pointed out, the proof does give an explicit estimate for $K$. The proof also shows that $X$ normalizes $S$. Laci Pyber remarked that with this strengthening (but not without it), the conclusion implies small tripling for $X$.

Here and later on, when confusion can arise between an iterated set product and a Cartesian power, we use $Y^{l}$ to denote the former, and $Y^{(l)}$ for the latter.

The assumption of Corollary 1.2 may be strong in a general group-theoretic setting, but it does hold for sufficiently dense subgroups of simple linear groups. The proof uses an idea originating in the Larsen-Pink classification of large finite simple linear groups, 35, somewhat generalized and formulated as a dimensioncomparison lemma in 24. We obtain: 
Theorem 1.3. Let $G$ be a semisimple algebraic group, defined over $\mathbb{Z}$, and $k$ an integer. Then for some integer $k^{\prime}$, the following statement holds. Let $K$ be a field, $X$ a finite subset of $G(K)$ with $\left|X X^{-1} X\right| \leq k|X|$. Then there exists a subgroup $H$ of $G(K)$ such that $|X / H| \leq k^{\prime}$, and either $H$ is (the set of $K$-points of) a connected proper algebraic subgroup of $G$ of degree $\leq k^{\prime}$, or $H \subseteq\left(X^{-1} X\right)^{2}$.

Here "degree $\leq k^{\prime \prime}$ " means that if we view $G$ as a subset of the $n \times n$ matrices $M_{n}$, then $H_{i}$ is the intersection of $G$ with a subvariety of $M_{n}$ cut out by polynomials of degree $\leq k^{\prime}$. Thus if the group generated by $X$ is sufficiently Zariski dense, $X$ will not be contained in such an algebraic subgroup, so that $X^{-1} X$ must be commensurable to a subgroup. A special case:

Corollary 1.4. For any $n \in \mathbb{N}$, for sufficiently large $n^{\prime} \in \mathbb{N}$, the following holds. Let $X$ be a finite subset of $G L_{n}(K), K$ a field, with $|X| \geq n^{\prime}$. Assume $\left|X X^{-1} X\right| \leq$ $k|X|$, and that $X$ generates an almost simple group $S$. Then $\left(X X^{-1}\right)^{2}=S$.

Here $S$ is not assumed to be finite. "Almost simple" means: perfect, and simple modulo a center of bounded size. The proof also shows that $X X^{-1}$ contains $99 \%$ of the elements of $S$, and that $X X^{-1} X=S$; see the proof and remarks following Proposition 5.10 .

For $S=S L_{2}\left(F_{q}\right)$ and $S L_{3}\left(F_{p}\right)$, Theorem 1.3 follows from results of Helfgott 20], 21] and Dinai; for $G=S L_{2}(\mathbb{C})$ and $G=S L_{3}(\mathbb{Z})$, Theorem 1.3 follows from [7] and [11. These authors all make a much weaker assumption on a subset $X$ of a group, namely $\left|X X^{-1} X\right| \leq|X|^{1+\epsilon}$ for a small $\epsilon$. The combinatorial regime they work in is also meaningful model-theoretically (cf. Example 2.13), but we do not study it at present.

Stable group theory includes a family of related results; for instance, the group law may be given by a multi-valued or partial function. The partial case has antecedents in algebraic geometry, in Weil's group chunk theorem. A version of the partial case, including the Freiman approximate groups mentioned above, will be briefly noted in the paper. It is likely that the multi-valued case too admits finite combinatorial translations along similar lines.

In $\$ 2$ we introduce the model-theoretic setting and prove the independence theorem and the stabilizer theorem in a rather general context. In the presence of a $\sigma$-additive measure the stabilizer sounds close to Tao's noncommutative BalogSzemeredi-Gowers theorem ([50]), while the independence theorem is, in the finite setting, extremely close to the Komlós-Simonovitz corollary [32 to Szemeredi's lemma (as I realized recently while listening to a talk by M. Malliaris.) It is thus quite possible that combinatorialists can find other proofs of the results of $\$ 2$ and skip to the next section. I find the independent, convergent development of the two fields rather fascinating.

All the results we need from stability will be explicitly defined and proved. Theorem 1.1 (and the more detailed Corollary 4.15) are proved in \$4. The methods here are very close to [25]; however, we do not assume NIP. This is in line with a sequence of realizations in recent years that tools discovered first in the stable setting are in fact often valid, when appropriately formulated, for first-order theories in general. Theorem 1.3 is proved in $\S 5$.

$\S 6$ contains a proof that the topology on the associated Lie group is generated by the image of a definable family of definable sets. 
In $\S 7$, we use the techniques of this paper, along with Gromov's proof of the polynomial growth theorem, to show (for any $k$ ) that if a finitely generated group is not nilpotent-by-finite, it has a finite set of generators contained in no $k$-approximate subgroup.

1.5. Basic model theory: around compactness. We recall the basic setup of model theory, directed to a large extent at an efficient use of the compactness theorem. We refer the reader to a book such as [6], 37, [43] or the lecture notes in 41 for a fuller treatment. We assume knowledge of the definition of a first-order formula, and of the compactness theorem, asserting that a finitely satisfiable set of formulas is satisfiable in some structure.

Let $L$ be a fixed language, $T$ a theory, $M$ a model. We will occasionally use notation as if the language is countable (e.g. indices named $n$ ), but this will not be really assumed unless explicitly indicated. At all events for much of this paper, a language with a symbol for multiplication and an additional unary predicate will be all we need.

$A$ will refer to a subset of $M$. We will assume $L, A$ are countable (this is quite inessential, and will be used only to avoid the need for cardinal parameters in discussing saturation below). We expand $L$ to a language $L(A)$ with an additional constant symbol for each element of $A$. The $L$-structure $M$ is tautologically expanded to an $L(A)$-structure, and the result is still denoted $M$, by abuse of notation. $T(A)$ is the $L(A)$-theory of $M . L_{x}(A)$ denotes the Boolean algebra of formulas of $L(A)$ with free variables $x$, up to $T(A)$-equivalence. $S_{x}(A)=\operatorname{Hom}\left(L_{x}(A), 2\right)$ is the Stone space, or the space of types. A subset of $L_{x}(A)$ is finitely satisfiable if each finite subset has a common solution in $M$. A type in a variable $x$, over $A$, is a maximal finitely satisfiable subset of $L_{x}(A)$. For an element or tuple $a$ over a subset $A$ of a model $M, t p(a / A)=\{\phi(x) \in L(A): M=\phi(a)\}$; if $t p(a / A)=p$, we say that $a$ realizes $p$. An $A$-definable set is the solution set of some $\phi \in L(A)$. It is an easy corollary of the compactness theorem that every theory $T$ has models $\mathbb{U}$ with the following properties holding for every small substructure $B$ of $\mathbb{U}$. Here let us say that $B$ is small if $2^{|B|} \leq|\mathbb{U}|$.

(1) Saturation: Every type over $B$ is realized in $\mathbb{U}$.

(2) Homogeneity: For $c, d$ tuples from $M, t p(c / B)=t p(d / B)$ iff there exists $\sigma \in \operatorname{Aut}(M / B)$ with $\sigma(c)=d$.

(In fact (1) implies (2) if the generalized continuum hypothesis holds; moreover, in this case, $\mathbb{U}$ is determined up to isomorphism by $T$ and by $|\mathbb{U}|$, provided $T$ is complete.)

Given a complete theory $T$, we fix a model $\mathbb{U}$ of $T$ with the above properties and with $|\mathbb{U}| \gg \aleph_{0}$ (if it is not finite), and interpret definable sets as subsets of $\mathbb{U}^{n}$. We will occasionally consider elementary submodels $M$ of $\mathbb{U}$; these will just be referred to as models. We write $A \leq M$ to mean that $A$ is a substructure of $M$. As stated above, substructures $A$ will be taken to be countable; "small" would be enough.

A partial type over $A$ is any collection of formulas over $A$, in some free variable $x$, and closed under implication in the $L_{A}$-theory of $M$.

The solution sets $D$ of partial types $r$ (over various countable sets $A$ ) are called $\Lambda$ definable (read: $\infty$-definable) sets; so an $\Lambda$-definable set over $A$ is any intersection of $A$-definable sets. The correspondence $r \mapsto D$ is bijective, because of the saturation property (1) above. Complements of $\Lambda$-definable sets are called $\bigvee$-definable. An 
equivalence relation is called $\bigwedge$-definable if it has an $\bigwedge$-definable graph. It follows from saturation that an $\bigwedge$-definable set is either finite or has size $|\mathbb{U}|$; a $\bigvee$-definable set is either countable or has size $\mathbb{U}$; an $\bigwedge$-definable equivalence relation has either $\leq 2^{\aleph_{0}}$ classes or $|\mathbb{U}|$-classes. Since $|\mathbb{U}|$ is taken to be large, this gap lends sense to the notion of bounded size for sets and quotients at these various levels of definability.

Another consequence of countable saturation is that projections commute with countable decreasing intersections:

$$
(\exists x) \bigwedge_{i=1}^{\infty} \phi_{i}(x, y) \Longleftrightarrow \bigwedge_{i=1}^{\infty}(\exists x) \phi_{i}(x, y)
$$

provided that $\phi_{i+1}$ implies $\phi_{i}$ for each $i$. The condition on the left beginning with $(\exists x)$ seems to be stronger, but compactness assures that the weaker condition on the right suffices for the existence of $x$ in some model, and countable saturation implies that such an $x$ exists in the given model. In particular, the projection of an $\bigwedge$-definable set is $\Lambda$-definable. We will use this routinely in the sequel. Specifically, if $Q$ is an $\Lambda$-definable subset of a definable group (see below), then the product set $Q Q=\{x:(\exists y, z \in Q)(x=y z)\}$ is also $\bigwedge$-definable.

By a definable group we mean a definable set $G$ and a definable subset - of $G^{3}$, such that $(G(\mathbb{U}), \cdot(\mathbb{U}))$ is a group. An $\bigwedge$-definable subgroup is an $\bigwedge$-definable set which is a subgroup. It need not be an intersection of definable subgroups. We insert here a lemma that may clarify these concepts.

A subset of a set $X$ is relatively definable if it has the form $X \cap Z$ for some definable $Z$.

Lemma 1.6. Let $G$ be a definable group. Let $X$ be an $\bigwedge$-definable subset of $G, Y$ $a \bigvee$-definable subset of $G$, and assume that $X$ and $X \cap Y$ are subgroups of $G$, and that $X \cap Y$ has bounded index in $X$. Then $X \cap Y$ is relatively definable in $X$ and has finite index in $X$.

Proof. By compactness, $[X: X \cap Y]<\infty$; otherwise, one can find an infinite sequence $\left(a_{i}\right)$ of elements of $X$ such that $a_{i} a_{j}^{-1} \notin Y$ for $i \neq j$, but since these are $\bigwedge$-definable conditions, arbitrarily long sequences with the same property exist. So $X \cap Y$ has finitely many distinct cosets $C_{1}, \ldots, C_{n}$ in $X$. Note that $X \backslash C_{i}$ is $\bigwedge$-definable. Hence $C_{j}=\bigcap_{i \neq j}\left(X \backslash C_{j}\right)$ is $\bigwedge$-definable for each $j$. Since $X_{i}$ and $C \backslash X_{i}$ are $\Lambda$-definable, they are relatively definable in $X$.

A $\mathbb{U}$-definable set is $A$-definable iff it is $\operatorname{Aut}(\mathbb{U} / A)$-invariant. The same is true for $\bigwedge$-definable sets and for $\bigvee$-definable sets.

Types over $\mathbb{U}$ are also called global types.

A sequence $\left(a_{i}: i \in \mathbb{N}\right)$ of elements of $\mathbb{U}$ is called $A$-indiscernible if for any order-preserving map $f: u \rightarrow u^{\prime}$ between two finite subsets of $\mathbb{N}$, there exists an automorphism $\sigma$ of $\mathbb{U}$ fixing $A$, such that $\sigma\left(a_{i}\right)=a_{f(i)}$ for $i \in u$. The same applies to sequences of $n$-tuples. Using Ramsey's theorem and compactness, one shows that if $\left(b_{i}: i \in \mathbb{N}\right)$ is any sequence, then there exists an indiscernible sequence $\left(a_{i}: i \in \mathbb{N}\right)$ such that for any formula $\phi(x, y)$, if $\phi\left(b_{i}, b_{j}\right)$ holds for all $i<j$, then $\phi\left(a_{i}, a_{j}\right)$ holds for all $i<j$. A theorem of Morley's [39] asserts the same thing with the formulas $\phi(x, y)$ replaced by types, provided $\mathbb{N}$ is replaced with a sufficiently large cardinal. For certain points (outside the main line), we will use Morley's theorem as follows. Let $q$ be a global type, and construct a sequence $a_{i}$ inductively, letting $A_{i}=\left\{a_{j}: j<i\right\}$, and choosing $a_{i}$ such that $a_{i} \models q \mid A_{i}$. By 
Morley's theorem, there exists an indiscernible sequence $\left(b_{0}, b_{1}, \ldots\right)$ such that for any $n, b_{n} \models q_{n} \mid\left\{b_{0}, \ldots, b_{n-1}\right\}$ for some Aut(UU)-conjugate $q_{n}$ of $q$.

We will say in this situation that $\left(b_{0}, b_{1}, \ldots\right)$ are $q$-indiscernibles. The main case is that $q$ is an invariant type, and then Morley's theorem is not needed, for the original $\left(a_{j}\right)$ are automatically indiscernible; see 42. A global type finitely satisfiable in $M$ is always $M$-invariant. In particular, given any type over $M$, this yields an $M$-indiscernible sequence $\left(a_{i}\right)$ such that $t p\left(a_{i} / M \cup A_{i}\right)$ does not fork over $M$ (cf. 22.1 for the definition). We remark that Morley's theorem uses more infinite cardinals than the rest of the paper (namely, not only infinite sets but arbitrary countable iterations of the power set operation).

In all notation, if $A$ is absent we take $A=\emptyset$. Generally a statement made for $T_{A}$ over $\emptyset$ is equivalent to the same statement for $T$ over $A$, so no generality is lost.

We will occasionally refer to ultraproducts of a family $M_{i}$ of $L$-structures. They are a specific way of constructing models $M$ of the set of all sentences holding in all but finitely many $M_{i}$, and they have the saturation property (1). No other properties of ultraproducts will be needed.

\section{INDEPENDENCE THEOREM}

2.1. Stability. The material in this subsection is a presentation of 31], Lemma 3.3, here Lemma 2.3. compare also [4], $\S 3$, and the stability section in [1.

Let $T$ be a first-order theory, $\mathbb{U}$ a universal domain. One of the main lessons of stability is the usefulness of $A$-invariant types, meaning $\operatorname{Aut}(\mathbb{U} / A)$-invariant types. We note that if a global type $p$ is finitely satisfiable in some $A \leq M$, then $p$ is $A$-invariant; if $a, a^{\prime}$ are Aut $(\mathbb{U} / A)$-conjugate, then $\phi(x, a) \& \neg \phi\left(x, a^{\prime}\right)$ cannot be satisfied in $A$.

We say that $A$ is an elementary submodel of $\mathbb{U}$ (written: $A \prec \mathbb{U}$ ) if any nonempty $A$-definable set has points in $A$. If $A \prec \mathbb{U}$, then any type over $A$ extends to a global type, finitely satisfiable in $A$ (41]).

Consider two partial types $r(x, y), r^{\prime}(x, y)$ over $A$. We say that $r, r^{\prime}$ are stably separated if there is no sequence $\left(\left(a_{i}, b_{i}\right): i \in \mathbb{N}\right)$ such that $r\left(a_{i}, b_{j}\right)$ holds for $i<j$, and $r^{\prime}\left(a_{i}, b_{j}\right)$ holds for $i>j$. Note that if arbitrarily long such sequences exist, then by compactness an infinite one exists, and in fact one can take the $\left(a_{i}, b_{i}\right)$ to form an $A$-indiscernible sequence. Moreover $r, r^{\prime}$ are stably separated iff they contain formulas $\phi, \phi^{\prime}$ that are stably separated. By reversing the ordering one sees that stable separation is a symmetric property.

We say that $r^{\prime}$ is equationally separated from $r$ if there is no sequence $\left(\left(a_{i}, b_{i}\right)\right.$ : $i \in \mathbb{N})$ such that $r\left(a_{i}, b_{i}\right)$ holds for all $i$, and $r^{\prime}\left(a_{i}, b_{j}\right)$ holds for $i<j$. This is an asymmetric condition, which implies stable separation: if stable separation fails, so that $r\left(a_{i}, b_{j}\right)$ holds for $i<j$, and $r^{\prime}\left(a_{i}, b_{j}\right)$ holds for $i>j$ in some sequence $\left(a_{i}, b_{i}\right)$, the shifted subsequence $\left(a_{2 i}, b_{2 i-1}\right)$ shows that equational separation fails too.

If $r, r^{\prime}$ are stably separated, then they are mutually inconsistent, since if $r(a, b)$ and $r^{\prime}(a, b)$ we can let $a_{i}=a, b_{j}=b$. In stable theories, the converse holds.

Note that the set of stably separated pairs is open in the space $S_{2}^{2}$ of pairs of 2 -types. Any extension of a stably separated pair to a larger base set remains stably separated.

A partial type $r^{\prime}(x, b)$ is said to divide over $A$ if there exists an indiscernible sequence $b_{0}, b_{1}, \ldots$ over $A$ such that $\bigcup_{i} r^{\prime}\left(x, b_{i}\right)$ is inconsistent, and $t p(b / A)=$ 
$t p\left(b_{i} / A\right)$. Equivalently, for some $k,\left\{r^{\prime}\left(x, b_{i}\right): i \in w\right\}$ is inconsistent for any $k$ element subset $w$ of $\mathbb{N}$. By compactness, $r^{\prime}(x, b)$ divides over $A$ iff some formula $R(x, b) \in r^{\prime}(x, b)$ divides over $A$. The ideal generated by all formulas that divide over $A$ is called the forking ideal; thus $\phi(x, c)$ forks over $A$ if it implies a disjunction of formulas that divide over $A$.

If $q=q(y)$ is a global type, we say that $r^{\prime}(x, y) q$-divides over $A$ if for some $n$, if $b_{i} \models q \mid A\left(b_{0}, \ldots, b_{i-1}\right)$ for $i \leq n$, then $\bigcup_{i \leq n} r^{\prime}\left(x, b_{i}\right)$ is inconsistent. This is equivalent to dividing, with the additional requirement that the indiscernible sequence be $q$-indiscernible.

Lemma 2.2. Let $r, r^{\prime}$ be stably separated formulas over $A$. Let $q(y)$ be an $A$ invariant global type. Assume $r^{\prime}(a, y) \in q, p=t p(a / A)$. Then $p(x) \cup r(x, y)$ $q$-divides over $A$.

Proof. Suppose it does not.

Define $a_{1}, \ldots, c_{1}, \ldots$ inductively: given $a_{1}, \ldots, a_{n-1}, c_{1}, \ldots, c_{n-1}$, choose $c_{n}$ such that $c_{n} \models q \mid\left\{a_{1}, \ldots, a_{n-1}, c_{1}, \ldots, c_{n-1}\right\}$, and $a_{n} \models p$ chosen with $r\left(x, c_{i}\right)$ for $i<n$. The latter choice is possible since $p(x) \cup r(x, y)$ does not $q$-divide over $A$.

Then $r^{\prime}\left(a_{i}, c_{j}\right)$ holds if $i<j$, but $r\left(a_{i}, c_{j}\right)$ holds when $i>j$. This contradicts the stable separation of $r, r^{\prime}$.

We say that an $A$-invariant relation $R$ is a stable relation over $A$ if whenever $(a, b) \in R$ and $\left(a^{\prime}, b^{\prime}\right) \notin R, t p((a, b) / A)$ and $t p\left(\left(a^{\prime}, b^{\prime}\right) / A\right)$ are stably separated. If $R$ is stable, so is the complement of $R$; but we are interested mostly in $\bigwedge$-definable $R$.

We will also encounter the condition of equationality. $R$ is equational if whenever $(a, b) \in R$ and $\left(a^{\prime}, b^{\prime}\right) \notin R, t p\left(\left(a^{\prime}, b^{\prime}\right) / A\right)$ is equationally separated from $t p((a, b) / A)$. As we have seen that equational separation implies stable separation, equationality implies stability.

We will say: " $R(a, b)$ holds" for " $(a, b) \in R$ ". When $q$ is a global type, write " $R(a, y) \in q(y)$ " to mean: $R(a, b)$ holds when $b \models q \mid A(a)$.

Lemma 2.3. Let $p(x)$ be a type over $A$, and $q(y)$ be a global, A-invariant type. Let $R$ be a stable relation over $A$.

(1) Assume $R(a, b)$ holds with $a \models p, b \models q \mid A(a)$. Then $R\left(a^{\prime}, b\right)$ holds whenever $a^{\prime} \models p$ and $\operatorname{tp}\left(a^{\prime} / A b\right)$ does not divide over $A$.

(2) Assume $\operatorname{tp}(a / A)=\operatorname{tp}\left(a^{\prime} / A\right), b \models q$, and neither $\operatorname{tp}(a / A b)$ nor $\operatorname{tp}\left(a^{\prime} / A b\right)$ divides over $A$. Then $R(a, b)$ implies $R\left(a^{\prime}, b\right)$.

(3) Assume $p$ too extends to a global, A-invariant type. Let $E=\{(a, b): a \models$ $p, b \models q \mid A\}$. Then the eight conditions:

$R(a, b)$ holds for some/all pairs $(a, b) \in E$ such that $t p(a / A(b)) / \operatorname{tp}(b / A(a))$ does not fork / divide over $A$

are all equivalent.

Proof. (1) Suppose $R\left(a^{\prime}, b\right)$ fails to hold. So $t p\left(a^{\prime}, b\right)$ and $t p(a, b)$ are stably separated, say by formulas $r^{\prime}, r$. By Lemma 2.2. since $r$ holds for $b \models q \mid A(a)$, $r^{\prime}(x, b) \cup p(x)$ divides, so $\operatorname{tp}\left(a^{\prime} / A b\right)$ divides over $A$, a contradiction.

(2) Let $R^{\prime}$ be the complement of $R$; it is also a stable relation. Let $c \models q \mid A(a)$. If $R(a, c)$ holds, then by (1) we have $R\left(a^{\prime}, b\right)$ and $R(a, b)$. If $R(a, c)$ holds, then similarly $R^{\prime}\left(a^{\prime}, b\right)$ and $R^{\prime}(a, b)$. In any case we have $R(a, b) \Longleftrightarrow R\left(a^{\prime}, b\right)$, so the stated implication holds. 
(3) Let $E^{\prime}$ be the set of pairs $(a, b) \in E$ such that $t p(a / A(b))$ does not divide over $A$, and $E^{\prime \prime}$ the set of pairs $(a, b) \in E$ such that $t p(b / A(a))$ does not divide over $A$. The equivalence between the four conditions for $t p(a / A(b))$ follows from (2): if $R(a, b)$ holds for some pair such that $t p(a / A(b))$ does not fork, then in particular it holds for a pair in $E^{\prime}$ (the same pair); by (2), it holds for all such pairs; hence certainly for all pairs for which $\operatorname{tp}(a / A(b))$ does not fork over $A$.

Thus a single truth value for $R$ is associated with pairs $(a, b) \in E^{\prime}$. Similarly, as the conditions are symmetric, a single truth value for $R$ is associated with pairs $(a, b) \in E^{\prime \prime}$. It remains to show that these truth values are equal. Replacing $R$ by its complement if necessary, we may assume $R(a, b)$ holds in the situation of (1), where $b \models q \mid A(a)$. In particular $t p(b / A(a))$ does not fork over $A$; so $R\left(a^{\prime}, b^{\prime}\right)$ holds for all $\left(a^{\prime}, b^{\prime}\right) \in E^{\prime \prime}$. But (1) asserts that $R\left(a^{\prime}, b\right)$ holds for all $\left(a^{\prime}, b\right) \in E^{\prime}$. Hence $R$ holds for all pairs in $E^{\prime} \cup E^{\prime \prime}$.

Nondividing in Lemma 2.3 (3) can be replaced by any stronger condition; nonforking was mentioned above; we will later use smaller ideals.

Remark 2.4. Let $p, q, R$ be as in Lemma 2.3, with $R(x, y)$ equational. Let $Q=$ $\{b: b \models q \mid A\}, P=\{a: a \models p \mid A\}$. If $R(a, b)$ holds with $a \models p, b \models q \mid A(a)$, then $P \times Q \subset R$.

Lemma 2.5. Let $S=S_{z}^{n f}$ be the set of global types that do not fork over $\emptyset$. Define an equivalence relation $E=E_{\text {st }}$ on $S: p E_{\text {st }} p^{\prime}$ iff for any stable invariant relation $R$, and any $b$, we have $R(b, z) \in p \Longleftrightarrow R(b, z) \in p^{\prime}$. Then $|S / E| \leq 2^{|T|}$.

Proof. Let $M$ be a model. It suffices to show that if $p\left|M=p^{\prime}\right| M$, then $p E_{s t} p^{\prime}$. Let $R(x, z)$ be a stable relation. Let $q=t p(b / M)$, and let $q^{*}$ be any $M$-invariant global type extending $q$. Let $c \models p \mid M$. By Lemma 2.3. since $p, p^{\prime}$ do not fork over $M$, $R(b, z) \in p$ iff $R(x, c) \in q^{*}$ iff $R(b, z) \in p^{\prime}$.

2.6. Making measures definable. A Keisler measure $\mu_{x}$ is a finitely additive real-valued probability measure on the formulas (or definable sets) $\phi(x)$ over the universal domain $\mathbb{U}$. See 25 .

We say $\mu$ is $A$-invariant if for any formula $\phi(x, y)$, for some function $g: S_{y}(A) \rightarrow$ $\mathbb{R}$, we have $\mu(\phi(x, b))=g(t p(b / A))$ for all $b$. If in addition $g$ is continuous, we say that $\mu$ is an $A$-definable measure.

Let $M_{i}$ be a family of finite $L$-structures. We wish to expand $L$ to a richer language $L[\mu]$, such that each $L[\mu]$ structure admits a canonical definable measure $\mu$. For each formula $\phi(x, y)$ and $\alpha \in \mathbb{Q}$ we introduce a formula $\theta(y)=\left(Q_{\alpha} x\right) \phi(x, y)$ whose intended intepretation is: $\theta(b)$ holds iff $\mu_{x} \phi(x, b) \leq \alpha$. If we wish $\mu$ to measure new formulas as well as $L$-formulas, this can be iterated.

We can expand each $M_{i}$ canonically to $L[\mu]$, interpreting the formulas $\left(Q_{\alpha} x\right) \phi(x, y)$ recursively using the counting measure.

Let $N$ be any model of the set of sentences true in all $M_{i}$ (such as the ultraproduct of the $M_{i}$ with respect to some ultrafilter). Define $\mu \phi(x, b)=\inf \{\alpha \in$ $\left.\mathbb{Q}:\left(Q_{\alpha} x\right) \phi(x, b)\right\}$. Then $\mu$ is a Keisler measure. The formulas $\left(Q_{\alpha} x\right) \phi$ may not have their intended interpretation with respect to $\mu$ exactly, but very nearly so: $\left(Q_{\alpha} x\right) \phi(x, b)$ implies $\mu_{x} \phi(x, b) \leq \alpha$, and is implied by $\mu_{x} \phi(x, b)<\alpha$. Thus $\mu$ is a definable measure on $N$.

We will actually only use the corollary that the 0 -ideal of $\mu$ is an invariant ideal; see below. 
2.7. Ideals. Let $X$ be a definable set, over $A$.

$L_{X}(\mathbb{U})$ denotes the Boolean algebra of $\mathbb{U}$-definable subsets of $X$. An ideal $I$ of this Boolean algebra is $A$-invariant if it is $\operatorname{Aut}(\mathbb{U} / A)$-invariant; equivalently $I$ is a collection of formulas of the form $\left\{\phi(x, a): t p(a / A) \in E_{\phi}\right\}$, where for each $\phi(x, y)$, $E_{\phi}$ is a subset of $S_{y}(A)$, and $\phi(x, a)$ implies $x \in X$. To emphasize the variable, we use the notation $I_{x}$.

We say that $I$ is $\bigwedge$-definable if for any $\theta(x, y)$, the set $\{b: \theta(x, b) \in I\}$ is $\bigwedge^{\text {- }}$ definable, and similarly for $\bigvee$-definable.

We say that a partial type $Q$ over $A$ is $I$-wide if it implies no formula in $I$.

In case $X$ is $\bigvee$-definable, i.e. a countable union of $A$-definable sets $X=\bigvee_{i} X_{i}$, we let $L_{x}(\mathbb{U})=\bigcup_{i} L_{X_{i}}(\mathbb{U})$. An ideal of $L_{x}(\mathbb{U})$ is a subset $I$ such that $I \cap L_{X_{i}}(\mathbb{U})$ is an ideal for each $i$; it is called $A$-invariant, $\bigwedge$-definable or $\bigvee$-definable if $I \cap L_{X_{i}}(\mathbb{U})$ has the corresponding property, for each $i$.

By analogy with measures, we will sometimes denote ideals in a variable $x$ by $\mu$, and write $\mu(\phi)=0$ for $\phi \in \mu$, and $\mu(\phi)>0$ for $\phi \notin \mu$.

The following definition is the defining property of $S 1$-rank, 23, relativized to an arbitrary ideal (so within a definable set of finite S1-rank, the definable sets of smaller S1-rank form an S1-ideal). The terms invariant, formula, indiscernible are understood over some fixed base set $A$.

Definition 2.8. An invariant ideal $I=I_{x}$ on $X$ is $S 1$ if for any formula $D(x, y)$ and indiscernible $\left(a_{i}: i \in \mathbb{N}\right)$ with $D\left(x, a_{i}\right) \in L_{X}(\mathbb{U})$, if $D\left(x, a_{i}\right) \cap D\left(x, a_{j}\right) \in I$ for $i \neq j$, then some $D\left(x, a_{i}\right) \in I$.

The forking ideal is contained in any S1-ideal:

Lemma 2.9. Let $I$ be an invariant $S 1$ ideal over $A$. If $\phi(x, b)$ forks over $A$, then $\phi(x, b) \in I$.

Proof. It suffices to show that if $\phi(x, b)$ divides over $A$, then $\phi(x, b) \in I$. Let $\left(b_{i}\right)$ be an $A$-indiscernible sequence, with $\left\{\phi\left(x, b_{i}\right)\right\}$ inconsistent; so for some $k$, $\phi\left(x, b_{1}\right) \wedge \ldots \wedge \phi\left(x, b_{k}\right)=\emptyset$. If $\phi\left(x, b_{1}\right) \in I$ we are done. Otherwise let $m$ be maximal such that $\phi\left(x, b_{1}\right) \wedge \ldots \wedge \phi\left(x, b_{m}\right) \notin I$. Let $c_{i}=\left(b_{1}, \ldots, b_{m-1}, b_{m+i}\right)$, and let $\psi\left(x, c_{i}\right)=\phi\left(x, b_{1}\right) \wedge \ldots \wedge \phi\left(x, b_{m-1}\right) \wedge \phi\left(x, b_{m+i}\right)$. Then the intersection of any two $\psi\left(x, c_{i}\right)$ is in $I$, but no $\psi\left(x, c_{i}\right)$ is in $I$. This contradicts Definition 2.8

The forking ideal over $A$ is also invariant under all $A$-definable bijections; in particular, for subsets of a group $G$ under left and right translations by elements of $G(A)$, i.e. by elements of $G$ definable over $A$. This will not be of real use to us, however, as we will be interested in translation invariance, right and left, by elements not necessarily defined over $A$.

The following is a fundamental observation from [8], 23], and [31]:

Lemma 2.10. Let $I_{z}$ be an invariant $S 1$-ideal. Let $P=P(x, z), Q=Q(y, z)$ be formulas. Define:

$$
R(a, b) \Longleftrightarrow(P(a, z) \wedge Q(b, z)) \in I_{z} .
$$

Then $R$ is a stable invariant relation.

Proof. We show indeed that $R$ is equational: if $R\left(a_{i}, b_{j}\right)$ holds for $i<j$, where $\left(a_{i}, b_{i}\right)_{i}$ is indiscernible, then $R\left(a_{i}, b_{i}\right)$ holds too.

Otherwise, let $C_{i}=\left\{z: P\left(a_{i}, z\right) \wedge Q\left(b_{i}, z\right)\right\}$. Then $C_{i} \notin I_{z}$, but $\mu_{z}\left(C_{i} \cap C_{j}\right)=0$. This contradicts the $\mathrm{S} 1$ property of Definition 2.8 . 
Example 2.11. Let $\mu(z)$ be a Keisler measure on $\mathbb{U}$-definable subsets of a set $Z$, with $\mu(Z)=1$. Let $e \in \mathbb{N}, \epsilon=1 / e>0$. Let $\phi(x, z), \phi^{\prime}(y, z)$ be formulas, and write $D(a, b)=\left\{z \in Z: \phi(a, z) \cap \phi^{\prime}(b, z)\right\}$. Let $r(x, y), r^{\prime}(x, y)$ be formulas such that if $r(a, b)$, then $\mu(D(a, b)) \geq \epsilon$, while $r^{\prime}(a, b)$ implies $\mu(D(a, b))<\epsilon^{2} / 2$. Then $r, r^{\prime}$ are stably separated; indeed $r$ is equationally separated from $r^{\prime}$. For suppose $r^{\prime}\left(a_{i}, b_{j}\right)$ holds for $i=1, \ldots, 2 e$. Let $D_{i}=D\left(a_{i}, b_{i}\right)$. Then $\mu\left(D_{i}\right) \geq \epsilon$, but $\mu\left(\bigcup_{1 \leq i<j \leq 2 e} D_{i} \cap D_{j}\right)<(2 e(2 e-1) / 2)\left(\epsilon^{2} / 2\right)<1$. So $\mu\left(\bigcup_{i} D_{i}\right)>2 e \epsilon-1=1$, a contradiction.

Example 2.12. Let $\mu$ be an $\operatorname{Aut}(\mathbb{U} / A)$-invariant, real-valued, finitely additive measure on $\mathbb{U}$-definable sets. Then $I=\{\phi(x, b): \mu(\phi(x, b))=0\}$ is an $\operatorname{Aut}(\mathbb{U} / A)$ invariant S1-ideal. It is $\Lambda$-definable if $\mu$ is definable.

Example 2.13. Let $X$ have nonstandard finite size $\alpha$, and let $I$ be the ideal of all definable sets with nonstandard size $\beta$, where $\log (\beta) \leq(1-\epsilon) \log (\alpha)$ for some standard $\epsilon>0$. (See $\S 5$ for detailed definitions.) Then $I$ is a $\bigvee$-definable ideal. It is not $\mathrm{S} 1$; but the counterexamples are always families contained in a definable set of dimension $<\epsilon \log (\alpha)$ for each $\epsilon>0$.

2.14. Wide global types. We now note the existence of useful global types relative to an ideal $I$, in three slightly different situations. The combinatorial applications of the present paper can be deduced from either Lemma 2.16 or Lemma 2.17. the former has a shorter, more general, but much more impredicative proof.

Lemma 2.15. Let $I=I(x)$ be a $\bigvee$-definable ideal, defined over a model $M$. Then there exists a global type $p$, finitely satisfiable in $M$, such that if $b \models p \mid M, a \models$ $p \mid M(b)$, then $\operatorname{tp}(b / M a)$ is I-wide. (In fact, whenever $p$ is finitely satisfiable in $M$ and $p \mid M$ is wide, then $p$ has this property.)

Proof. Let $p_{0}$ be any wide type over $M$, and let $p$ be any extension to $\mathbb{U}$, finitely satisfiable in $M$. Let $b=p|M, a=p| M(b)$. If $t p(b / M a)$ is not wide, then for some $\phi(x, y)$ we have $\phi(a, b)$ and $\phi(a, y) \in I$; by $\bigvee$-definability, for some $\theta \in t p(a / M)$, for all $a^{\prime}$ with $\theta\left(a^{\prime}\right), \phi\left(a^{\prime}, y\right) \in I$. Since $t p(a / M b)$ is finitely satisfiable in $M$, there exists $a^{\prime} \in M$ with $\theta\left(a^{\prime}\right)$ and $\phi\left(a^{\prime}, b\right)$. It follows that $p_{0}=t p(b / M)$ is not wide, a contradiction.

Lemma 2.16. Let $I=I(x)$ be an A-invariant ideal. There exists a model $M \geq A$, a global $M$-invariant type $q$, finitely satisfiable in $M$, such that if $a \models q \mid M$ and $b \models q \mid M(a)$, then $\operatorname{tp}(a / M(b))$ is wide.

Proof. Let $T_{s k}$ be a Skolemization of the theory, in an expansion $L_{s k}$ of the language $L$; so the $L_{s k}$-substructure $M(X)$ generated by a set $X$ is an elementary submodel. Define a sequence of elements $a_{i}\left(i<\beth_{\omega_{1}}\right)$, and sets $A_{i}=M\left(\left\{a_{j}: j<i\right\}\right)$, with $t p_{L}\left(a_{i} / A_{i}\right)$ wide. By Morley's theorem [39], there exists an indiscernible sequence $\left(c_{i}: i<\omega+2\right)$ such that for any $n$, for some $i_{1}<\ldots<i_{n}, t p\left(c_{1}, \ldots, c_{n}\right)=$ $\operatorname{tp}\left(a_{i_{1}}, \ldots, a_{i_{n}}\right)$. In particular, $t p\left(c_{i} /\left\{c_{j}: j<i\right\}\right)$ is wide. Let $U$ be an ultrafilter on $\mathbb{N}$, and let $q$ be the set of formulas $\phi(x)$ of $L(\mathbb{U})$ such that $\left\{i: \phi\left(c_{i}\right)\right\} \in U$. Let $M=A_{\omega}$. Then $q$ is finitely satisfiable in $M$. Let $a=c_{\omega+1}, b=c_{\omega}$. Then $a \models q \mid M$ and $b=q \mid M(a)$, and $t p(a / M(b))$ is wide.

Lemma 2.17. Let $I=I(x)$ be an $\Lambda$-definable ideal, defined over a model $M$ with $L(M)$ countable. Assume ("Fubini") there exists an ideal $I^{2}(x, y)$ on $L_{x, y}(M)$ such that: (i) if $\phi(a, y) \in I(y)$ whenever $t p(a / M)$ is I-wide, then $\phi \in I^{2}$; (ii) if 
$\phi(x, b) \in I(x)$ whenever $t p(b / M)$ is $I$-wide, then $\phi \in I^{2}$; (iii) if $\phi(x) \wedge \phi(y) \in I^{2}$, then $\phi \in I$.

Then there exists a global type $p$, finitely satisfiable in $M$, such that if $b=p \mid M$, $a \models p \mid M(b)$, then $\operatorname{tp}(a / M b)$ and $t p(b / M a)$ are $I$-wide.

Proof. Let $B$ be the Boolean algebra of formulas of $M$ modulo $I$. We show that a generic ultrafilter $p_{0}$ on $B$ (in the sense of Baire category) can be extended to a type satisfying the lemma.

Claim. Let $\phi_{i}(x, y)(i=1,2,3)$ be a triple of formulas, and let $P(x) \in B \backslash I$. Assume

$$
P(x) \wedge P(y) \vdash \bigvee_{i=1}^{3} \phi_{i}(x, y) .
$$

Then for some $P^{\prime} \in B \backslash I$ implying $P$, for any $a, b \in P^{\prime}$, we have $\left(^{*}\right): \quad \phi_{1}(a, y) \notin I$ or $\phi_{2}(x, b) \notin I$ or $\phi_{3}(c, b)$ for some $c \in M$.

Proof. If $\left(P(x) \wedge \phi_{3}(c, x)\right) \notin I$ for some $c \in M$, we can let $P^{\prime}(x)=P(x) \wedge \phi_{3}(c, x)$; then the third option in $\left(^{*}\right)$ is met. Otherwise, $\left(P(x) \wedge \phi_{3}(c, x)\right) \in I$ for all $c \in M$. It follows from the $M$-^-definability of $I$ that $\left(P(x) \wedge \phi_{3}(c, x)\right) \in I$ for all $c$. So $P(y) \wedge \phi_{3}(x, y) \in I^{2}$.

If for some $P^{\prime} \in B \backslash I$ implying $P$ we have: $P^{\prime}(a)$ implies $\phi_{1}(a, x) \notin I$, then the first disjunct of $\left(^{*}\right)$ holds. Otherwise, using the $M$ - $\bigwedge$-definability of $I$, we see that for all $a \in P$ with $\operatorname{tp}(a / M) I$-wide, $\phi_{1}(a, y) \in I$. By the Fubini assumption (i), $P(x) \wedge \phi_{1}(x, y) \in I^{2}$.

Similarly, if for some such $P^{\prime}, P^{\prime}(b)$ implies $\phi_{2}(x, b)>0$, then the second disjunct holds. Otherwise, by Fubini (ii), $\left(\phi_{2}(x, y) \wedge P(y)\right) \in I^{2}$.

Since $P(x) \wedge P(y)$ implies the disjunction of the $\phi_{i}$, we have $(P(x) \wedge P(y)) \in I^{2}$; so $P \in I$, contradicting the choice of $P$, and proves the claim.

It is now easy to construct a type $p_{0}$ over $M$ such that, for any $\phi_{1}(x, y), \phi_{2}(x, y)$, $\phi_{3}(x, y)$, if $p_{0}(x) \cup p_{0}(y) \vdash \bigvee_{i=1}^{3} \phi_{i}(x, y)$, then $\left(^{*}\right)$ of the claim holds for any $a, b \models p_{0}$. Namely, we let $p_{0}=\left\{P_{n}\right\}$, where $P_{n} \in B \backslash I$ is constructed recursively. If $n$ is even, we choose $P_{n+1}$ so as to imply $\psi$ or $\neg \psi$, where $\psi$ is the $n / 2$-nd element of some enumeration of the formulas $\psi(x)$. If $n=2 m+1$ is odd, consider the $m$ th triple $\left(\phi_{1}, \phi_{2}, \phi_{3}\right)$ in some (infinitely repetitive) enumeration of all triples of formulas over $M$. If $P(x) \cup P(y) \vdash \bigvee_{i=1}^{3} \phi_{i}$, let $P^{\prime}$ be as in the claim, and let $P_{n+1}=P_{n} \wedge P^{\prime}$.

Let $b \models p_{0}$, and let $\Gamma(x, b)=p_{0}(x) \cup\left\{\neg \phi_{1}(x, b): \phi_{1}(x, b) \in I\right\} \cup\left\{\neg \phi_{2}(x, b)\right.$ : $\left.\phi_{2}(b, x) \in I\right\} \cup\left\{\neg \phi_{3}(x, b):\left(\forall c^{\prime} \in M\right)\left(\phi_{3}\left(c^{\prime}, x\right) \notin p_{0}\right)\right\}$. If $\Gamma(x, b)$ is inconsistent, then $p_{0}(x) \cup p_{0}(y) \vdash \phi_{1}(x, b) \vee \phi_{2}(x, b) \vee \phi_{3}(x, b)$ for some $\phi_{1}, \phi_{2}, \phi_{3}$ with $\phi_{1}(x, b) \in$ $I, \phi_{2}(b, x) \in I, \phi_{3}$ such that $\left.\left(\forall c^{\prime} \in M\right)\left(\phi_{3}\left(c^{\prime}, x\right) \notin p_{0}\right)\right\}$. But this contradicts the construction of $p_{0}$. Thus $\Gamma(x, b)$ is consistent, and in view of the formulas $\neg \phi_{3}$, finitely satisfiable in $M$. Let $p$ be any extension of $\Gamma(x, b)$ to a global type finitely satisfiable in $M$. Let $b \models p|M, a \models p| M(b)$. Then $t p(a / M b)$ is wide because of the formulas $\neg \phi_{1}$, and $t p(b / M a)$ is wide because of the formulas $\neg \phi_{2}$.

We now come to the 3 -amalgamation statement. It says roughly that given a triangle of types, an arbitrary replacement of one edge by another with the same vertices will not affect the wideness of the opposite vertex over the edge. To simplify notation we work over $A=\emptyset$, so "divides" means "divides over $\emptyset "$.

Theorem 2.18. Let $\mu=\mu_{z}$ be an invariant S1-ideal. Assume tp $(c / a, b)$ is $\mu_{z^{-}}$ wide, $t p(b / a)$ and $t p\left(b^{\prime} / a\right)$ do not divide, $t p(a)$ extends to an invariant global type, 
and $t p(b)=t p\left(b^{\prime}\right)$. Then there exists $c^{\prime}$ with $t p\left(c^{\prime} / a, b^{\prime}\right)$ wide, and $t p\left(c^{\prime} b^{\prime}\right)=$ $t p(c b), t p\left(c^{\prime} a\right)=t p(c a)$.

Proof. Let $Q \in t p(c b), P \in t p(c a)$. By compactness, it suffices, for any such pair of formulas, to find $c^{\prime}$ with $t p\left(c^{\prime} / a, b^{\prime}\right)$ wide, and $Q\left(c^{\prime}, b^{\prime}\right), P\left(c^{\prime}, a\right)$. In other words it suffices to show that $\mu_{z}\left(Q\left(z, b^{\prime}\right) \wedge P(z, a)\right)>0$.

Consider the relation $R(x, y)$ such that $R(d, e)$ holds iff $\mu_{z}(P(z, d) \wedge Q(z, e))=0$. By Lemma 2.10, it is a stable relation.

By assumption, $t p\left(b^{\prime} / a\right)$ and $t p(b / a)$ do not divide. By Lemma 2.3, since $R(a, b)$ fails, $R\left(a, b^{\prime}\right)$ must fail too. Thus $\mu_{z}(P(a, z) \wedge Q(b z))>0$.

Remark 2.19. (1) The hypothesis that $t p(b / a)$ and $t p\left(b^{\prime} / a\right)$ do not divide can be replaced by: $t p(b / a)$ and $t p\left(a / b^{\prime}\right)$ do not divide, using Lemma 2.3 (3).

(2) Over a model, the hypothesis that $t p(a)$ extends to an invariant global type holds automatically.

(3) If $E$ is an $\Lambda$-definable equivalence relation over $A$ with boundedly many classes, and the class of $a$ is not the unique wide class within $\operatorname{tp}(a)$, then 3 -amalgamation can fail; one cannot amalgamate a type $p(x, y)$ implying $\neg(x E y)$ with any types implying $x E y, y E z$. It is possible that this is the only obstruction, so that as in [31, Theorem 2.18 holds over any set $A$ which is boundedly closed.

2.20. Complements. In the remainder of this section we mention a variant of Theorem 2.18 in a measured setting, bringing out the 3-amalgamation aspect, and discuss connections to NIP and to probability theory. None of this will be needed for the combinatorial applications of $\S \S 2-5$.

An arbitrary triangle of 2-types cannot be expected to give a consistent 3-type, for instance since a definable linear ordering may be present; types including $x<$ $y, y<z, z<x$ are obviously not consistent together. But in a measured setting, contrary to initial appearances, this obstruction has an effect only on a measure zero set.

Below, $i$ ranges over elements of $\Upsilon:=\{1,2,3\}$, while $u$ ranges over subsets of $\Upsilon$ of size 2. Let $x_{i}$ be a sort, and let $X_{i}$ be the space of types in this sort, over a fixed base set $M$. We assume every type in $X_{i}$ extends to an invariant type (as is the case over an elementary submodel). We also assume, for simplicity's sake, that $L(M)$ is countable. For $i \in \Upsilon$ let $\mu_{i}$ be an $M$-definable measure on $X_{i}=X_{x_{i}}$. In fact it suffices to assume that $\mu_{i}$ is Borel-definable over $M$, meaning that $\mu_{i}(\phi(x, b))$ is a Borel function of $t p(b / M)$.

Assume the $\mu_{i}$ commute, in the sense that for any $i \neq j \in \Upsilon$, for any formula $\phi\left(x_{i}, x_{j}\right)$ over $M$,

$$
\int \mu_{j}\left(\phi\left(x_{i}, x_{j}\right)\right) d \mu_{i}=\int \mu_{i}\left(\phi\left(x_{i}, x_{j}\right)\right) d \mu_{j}
$$

see [27]. Any measures obtained as ultraproducts of counting measures will certainly have this property.

The common value is denoted by $\mu_{i j}(\phi)$; this defines a measure with variables $\left(x_{i}, x_{j}\right)$, referred to as the tensor product of $\mu_{i}, \mu_{j}$. Similarly, for $u \subseteq \Upsilon$, let $\mu_{u}$ be the tensor product measure on $X_{u}$. In particular we have $\mu=\mu_{123}$ on $X_{123}=X(\Upsilon)$.

We will occasionally refer to random elements; this can be given precise settheoretic foundations, but we will not do this here. Instead we will understand by 
this an element of a type space, or a product of type spaces, avoiding a certain countable collection of measure-zero Borel sets, which can be explicitly specified by inspecting the proof. We will also omit the foundational details of the notion of conditional measures, noting only that in the context of separable totally disconnected spaces we have a canonical countable Boolean algebra, namely the clopen subsets, making things easier.

Consider the natural maps $X(\Upsilon) \rightarrow X(\{23\}), X(\Upsilon) \rightarrow X(2) \times X(3)$, etc. For any such map, with target $Y$ carrying measure $\mu_{Y}$, and given a random (for the pushforward measure of $\mu$ ) element $y \in Y$, we let $X_{123}(y)$ denote $X_{123}$ with the measure $\mu_{123 / y}$ conditioned on $y$. These conditional measures concentrate on the fiber over $y$, and satisfy: $\mu(B)=\int\left(\mu_{123 / y}(B)\right) d \mu_{Y}$ for any clopen $B$. This formula defines $\mu_{123 / y}$ uniquely for a random $y$, in the sense that any two choices will agree for almost all $y$. Again we refrain from giving the foundational details, noting only that they are much easier in the present context of separable totally disconnected spaces; this is due to the availability of a canonical countable Boolean algebra generating the measure algebra, namely the clopen subsets. See 19].

We will consider formulas $\theta_{u}$ in variables $\left(x_{i}: i \in u\right)$, and let $\theta=\bigwedge_{|u|=2} \theta_{u}$. We interpret $\theta$ on the one hand as a clopen subset of $X_{\Upsilon}$, on the other hand as a clopen subset of $\Pi_{u} X_{u}$, namely $\Pi_{u} \theta_{u}$.

Lemma 2.21. Let $\left(q_{1}, q_{2}, q_{3}\right) \in \Pi_{i} X_{i}$ be a random triple. Let $q_{23}=t p\left(a_{2} a_{3} / M\right)$, where $\operatorname{tp}\left(a_{3} / M\left(a_{2}\right)\right)$ does not divide over $M$, and $\operatorname{tp}\left(a_{i} / M\right)=q_{i}(i=1,2)$. Let $\theta_{1 j}\left(x_{1}, x_{j}\right)$ be a formula of positive measure for $X_{1 j}\left(q_{1}, q_{j}\right)$ (the space $X_{1 j}$ with measure $\mu$ conditioned on $\left.\left(q_{1}, q_{j}\right)\right)$. Then $\theta_{12}\left(x_{1}, x_{2}\right) \wedge \theta_{13}\left(x_{1}, x_{3}\right) \cup q_{23}$ is consistent. In fact for $\left(a_{2}, a_{3}\right)=q_{23}, \theta_{12}\left(x_{1}, a_{2}\right) \wedge \theta_{13}\left(x_{1}, a_{3}\right)$ has positive $\mu_{1}$-measure.

Proof. Choose $p_{12} \in \theta_{12}$, random in $X_{12}\left(q_{1}, q_{2}\right)$ over $\left(q_{1}, q_{2}, q_{3}\right)$. Note that $p_{12}$ extends $q_{1}, q_{2}$. Since $q_{2}$ is random over $\left(q_{1}, q_{3}\right), p_{12}$ is random in $X_{12}\left(q_{1}\right)$ over $\left(q_{1}, q_{3}\right)$, and in $X_{12}$ over $\left(q_{3}\right)$. Hence $\left(q_{3}, p_{12}\right)$ are random in $X_{3} \times X_{12}$.

Choose $p_{13} \in \theta_{13}$, random in $X_{13}\left(q_{1}, q_{3}\right)$ over $\left(p_{12}, q_{3}\right)$. Again $p_{13}$ extends $q_{1}, q_{3}$, and (as $q_{3}$ is random over $\left(p_{12}\right)$ in $\left.X_{3}\right), p_{13}$ is random in $X_{13}\left(q_{1}\right)$ over $\left(p_{12}\right)$, so $\left(p_{12}, p_{13}\right)$ is random in $X_{12}\left(q_{1}\right) \times X_{13}\left(q_{1}\right)$ over $\left(q_{1}\right)$. Now the product measure on $X_{12}\left(q_{1}\right) \times X_{13}\left(q_{1}\right)$ coincides with the pushforward measure from $X_{123}\left(q_{1}\right)$. (This is best seen "over $q_{1}$ ".) So by choosing $p_{123}$ at random in $X_{123}\left(p_{12}, p_{13}\right)$ (with the conditional measure), we find $p_{123}$ containing $p_{12}, p_{13}$ and random. Let $p_{23}$ be the restriction of $p_{123}$ to the 2,3 -variables. Let $\left(b_{2}, b_{3}\right) \models p_{23}$. Note that $p_{23}$ is random in $X_{23}$, so $t p\left(b_{3} / M\left(b_{2}\right)\right)$ does not divide over $M$.

Now $\theta_{12}\left(x_{1}, b_{2}\right) \wedge \theta_{13}\left(x_{1}, b_{3}\right)$ has positive $\mu_{1}$-measure (otherwise $p_{123}$ could not be random). By Theorem 2.18, $\theta_{12}\left(x_{1}, a_{2}\right) \wedge \theta_{13}\left(x_{1}, a_{3}\right)$ has positive $\mu_{1}$-measure too.

Theorem 2.22. Assume $L(M)$ is countable. Let $\Upsilon=\{1,2,3\}$. For $i \in \Upsilon$ let $\mu_{i}$ be an $M$-definable measure on $X_{i}=X_{x_{i}}$, and assume the $\mu_{i}$ commute. For $u \subseteq \Upsilon$, $|u|=2$, let $\mu_{u}$ be the tensor product measure on $X_{u}$. Then there exist measure-one Borel subsets $\Omega_{u} \subset X_{u}$ and $\Omega \subset X_{1} \times X_{2} \times X_{3}$ with the following amalgamation property. Assume $q_{u} \in \Omega_{u},\left(q_{1}, q_{2}, q_{3}\right) \in \Omega, q_{u} \mid i=q_{i}$ for $i \in u$. Then there exists $q \in X_{\Upsilon}, q \mid u=q_{u}$.

In fact, we can take $\Omega_{23}$ to be the set of all tp $(b c)$ such that $t p(b / c)$ does not divide over $M$. 
Proof. It suffices to show that if $\left(q_{1}, q_{2}, q_{3}\right)$ is random, in $X_{1} \times X_{2} \times X_{3}, q_{u}$ is random in $X_{u}$ for $|u|=2$, and $q_{i} \subset q_{u}$ for $i \in u$, then there exists $q \in X_{\Upsilon}, q \mid u=q_{u}$. Fix such $q_{i}, q_{u}$. By compactness, it suffices to show for any given triple of formulas $\theta_{u} \in q_{u}$ that $\theta=\bigwedge_{u} \theta_{u}$ is consistent. Fix such a $\theta_{u}$. Since $q_{1 j}$ is random in $X_{1 j}$, it is random in $X_{1 j}\left(q_{1}, q_{j}\right)$ over $\left(q_{1}, q_{j}\right)$. Hence $\theta_{1 j}$ has positive measure in $X_{1 j}\left(q_{1}, q_{j}\right)$. By Lemma 2.21, even $\theta_{12}\left(x_{1}, x_{2}\right) \wedge \theta_{13}\left(x_{1}, x_{3}\right) \cup q_{23}$ is consistent.

Note that since $\Omega_{w}$ has measure 1 , for a random choice of $q_{i} \in X_{i}(i=1,2,3)$, one expects the existence of $q_{w} \in S_{w}(w \subset\{1,2,3\}$ with $|w|=2)$ with $q_{i} \subseteq q_{w}$ when $i \in w$. The (obviously necessary) hypothesis of compatibility on the $q_{w}$ is therefore frequently attained.

Thanks to Pierre Simon for his comments on this. This result admits a more precise numerical version, or alternatively a formulation using ideals, and a higherdimensional generalization; this and related issues will be taken up elsewhere.

\subsection{NIP and de Finetti.}

Example 2.24. Let $\mu$ be an $A$-definable Keisler measure in a NIP theory; cf. 26. Let $\phi(x, y), \phi^{\prime}(x, y)$ be formulas. For any real $\alpha$, let $R_{\alpha}(a, b)$ denote the relation: $\mu\left(\phi(x, a) \cap \phi^{\prime}(x, b)\right)<\alpha$. Then $R_{\alpha}$ is equational. This uses the fact that for an indiscernible sequence $\left(c_{j}\right)$ over $A$ we have $\mu\left(\psi\left(x, c_{i}\right) \cap \psi\left(x, c_{j}\right)\right)=\mu\left(\psi\left(x, c_{i}\right)\right)$, applied to $c=(a, b), \psi(x, c)=\phi(x, a) \wedge \phi^{\prime}(x, b)$.

When $\alpha>0$, the relation $\mu\left(\phi(x, a) \cap \phi^{\prime}(x, b)\right)=\alpha$ need not be equational, as one sees for instance by taking $\phi=\phi^{\prime}$ and an indiscernible sequence $\left(a_{i}, b_{i}\right)$ with $a_{i}=b_{i}$.

However, in any theory, we have:

Proposition 2.25. For any invariant measure $\nu$, the relation $\nu(\phi(x, a) \cap \psi(x, b))=$ $\alpha$ is stable. In other words, when $\left(a_{i}, b_{i}\right)$ is an indiscernible sequence of pairs, the function $(i, j) \mapsto \nu\left(\phi\left(x, a_{i}\right) \cap \psi\left(x, b_{j}\right)\right)$ is symmetric in $i, j$.

It follows that for any subset $Y$ of $[0,1]$, the relation: $\nu(\phi(x, a) \cap \psi(x, b)) \in Y$ is stable.

The proof is related to a classical theorem of de Finetti, classifying the so-called exchangeable sequences of random variables, i.e. sequences such that the action of the symmetric group does not change joint distributions. This was subsequently generalized by [22], 34- 33], and in a different direction by Aldous and Hoover; see [28. Thanks to Benjy Weiss for telling me about this theory. Though the assumption is classically stated as symmetry, indiscernibility suffices for the arguments; the proof below is essentially a subset of the one in 33] (in turn a modification of 22). The higher-dimensional case will be considered elsewhere.

Proof of Proposition 2.25. We show more generally that if $\left(a_{i}: i \in \mathbb{N}\right)$ is an indiscernible sequence, and $\psi_{1}, \ldots, \psi_{k}$ any formulas, then $\nu\left(\psi_{1}\left(x, a_{1}\right) \wedge \cdots \wedge \psi_{n}\left(x, a_{k}\right)\right)$ is invariant under the action of the symmetric group on $\left\{a_{1}, \ldots, a_{k}\right\}$, i.e.

$$
\mu\left(\psi_{1}\left(x, a_{1}\right) \cap \cdots \cap \psi_{k}\left(x, a_{k}\right)\right)=\mu\left(\psi_{1}\left(x, a_{\sigma 1}\right) \cap \cdots \cap \psi_{k}\left(x, a_{\sigma k}\right)\right)
$$

for any $\sigma \in \operatorname{Sym}(k)$.

Let $B(\mathbb{N})$ be the Boolean algebra generated by the formulas $\psi_{i}\left(x, a_{j}\right)$ for $i \leq$ $k, j \in \mathbb{N}$. Let $S=S(\mathbb{N})$ be the Stone space of $B(\mathbb{N})$. Let $\mathcal{M}$ be the space of countably additive regular Borel probability measures on $S(\mathbb{N})$. For a finite $J \subset \mathbb{N}$, 
let $B(J)$ be the subalgebra generated by the $\psi_{i}\left(x, a_{j}\right)$ with $j \in J, S(J)$ the Stone space, and for $\mu \in \mathcal{M}$, let $\mu \mid J$ be the induced measure, i.e. the pushforward of $\mu$ under the restriction map. Let $\mathcal{M}_{\text {ind }}$ be the subset of indiscernible measures, i.e. measures $\mu$ on $S$ such that for any finite $J_{1}, J_{2} \subset \mathbb{N}$ of the same size, with order-preserving bijection $j: J_{1} \rightarrow J_{2}$, the induced map $j: B\left(J_{1}\right) \rightarrow B\left(J_{2}\right)$ is measure-preserving, i.e. $j_{*}\left(\mu \mid J_{1}\right)=\mu \mid J_{2}$.

Let $\mathcal{M}_{\text {sym }}$ be the apparently smaller subset of symmetric (or exchangeable) measures, where we demand that $j_{*}\left(\mu \mid J_{1}\right)=\mu \mid J_{2}$ for any bijection $j: J_{1} \rightarrow J_{2}$.

Claim 1. $\mathcal{M}_{\text {sym }}=\mathcal{M}_{\text {ind }}$.

To prove the claim, note that both sets are convex and weak-* closed subsets of the unit ball of $\mathcal{M}$. Hence by Krein-Milman (cf. e.g. [57]), to show equality it suffices to prove that any extreme point of $\mathcal{M}_{\text {ind }}$ is in $\mathcal{M}_{\text {sym }}$. So assume $\mu$ is an extreme point of $\mathcal{M}_{\text {ind }}$. Now Claim 1 follows from:

Claim 2. When $\mu \in \mathcal{M}_{\text {ind }}$ is extreme, we have independence: $\mu\left(\phi_{1}\left(x, a_{1}\right) \wedge \cdots \wedge\right.$ $\left.\phi_{n}\left(x, a_{n}\right)\right)=\prod_{i=1}^{n} \mu \phi_{i}\left(x, a_{i}\right)$, for any $\phi_{i}\left(x, a_{i}\right) \in B\left(\left\{a_{i}\right\}\right)$.

Let $\alpha=\mu\left(\phi_{1}\left(x, a_{1}\right)\right)$. If $\alpha=0$, then $\mu\left(\phi_{1}\left(x, a_{j}\right)\right)=0$ for any $j$, and both sides of the equation vanish. If $\alpha=1$, then $\phi_{1}$ may be deleted on both sides, and the claim follows by induction on $k$. Assume therefore that $0<\alpha<1$. Let $\mu^{\prime}$ be obtained from $\mu$ by conditioning on $\phi_{1}\left(x, a_{1}\right)$, and shifting indices:

$$
\mu^{\prime}\left(\theta\left(x, a_{1}, \ldots, a_{m}\right)\right)=\mu\left(\theta\left(x, a_{2}, \ldots, a_{m+1}\right) \wedge \phi_{1}\left(x, a_{1}\right)\right) / \alpha .
$$

Similarly, let $\mu^{\prime \prime}$ be obtained from $\mu$ by conditioning on $\neg \phi_{1}\left(x, a_{1}\right)$, and shifting indices. Then $\mu=\alpha \mu^{\prime}+(1-\alpha) \mu^{\prime \prime}$, and $\mu^{\prime}, \mu^{\prime \prime} \in \mathcal{M}_{\text {ind }}$. As $\mu$ is extreme, we have $\mu=\mu^{\prime}$. This means:

$$
\mu\left(\phi_{1}\left(x, a_{1}\right) \wedge \theta\left(x, a_{2}, \ldots, a_{m}\right)\right)=\mu\left(\phi_{1}\left(x, a_{1}\right)\right) \mu\left(\theta\left(x, a_{2}, \ldots, a_{m}\right)\right) .
$$

Here $m, \theta$ are arbitrary. Claim 2 follows by induction on $m$, letting $\theta\left(x, a_{2}, \ldots, a_{n}\right)$ $=\phi_{2}\left(x, a_{2}\right) \wedge \cdots \wedge \phi_{n}\left(x, a_{n}\right)$.

Claim 1 follows easily: the right-hand side of the formula of Claim 2 is clearly symmetric. Any formula in $B(\mathbb{N})$ is a disjoint union of set-theoretic differences of conjunctions as considered in Claim 2. The measure of the difference of two such expressions can be computed using the inclusion-exclusion formula, and of disjoint unions by additivity.

Finally note that if $\nu$ is an invariant measure, indiscernibility of the $\left(a_{i}\right)$ implies indiscernibility of $\nu \mid B(\mathbb{N})$; hence the proposition follows from Claim 1.

\section{THE STABILIZER}

Let $G$ be a group, $X$ a subset, defined over some model $M_{0}$. Let $\widetilde{G}$ be the subgroup of $G$ generated by $X$ (cf. [25, $\S 7$ ). By a definable subset of $\widetilde{G}$, we mean a definable subset of $\left(X \cup X^{-1}\right)^{\leq n}$ for some $n$. A subset $Y$ of $\widetilde{G}$ is locally definable if $Y \cap D$ is definable for every definable subset $D$ of $\widetilde{G}$.

Remark 3.1. In sections 3 and 4 we will never use $G$, only $\widetilde{G}$. It is thus natural to use a many-sorted reduct, whose universes consist of the sets $\left(X \cup X^{-1}\right)^{\leq n}$, with the inclusion maps and multiplication maps between them, and a distinguished predicate for $X$. We will speak of the inclusion maps as if they were actual inclusions. 
Going further, we can note that we actually use only a bounded number of multiplications. In this section we will use only elements of $\left(X X^{-1}\right)^{3}$ and will only use associativity for products of at most twelve elements of $X$ and their inverses.

Hence the results of this section are valid for structures $\left(X, X^{\prime}, G\right)$ with $X \subset$ $X^{\prime} \subset G$, with a binary map $m:\left(X^{\prime}\right)^{2} \rightarrow G$ and an inversion map ${ }^{-1}: X^{\prime} \rightarrow X^{\prime}$, such that products of up to twelve elements of $X \cup X^{-1}$ are defined, and independent of order. We will refer to this as a "local group" situation (cf. [16]). In this case $\widetilde{G}$-translation invariance for a measure is replaced by the condition that $\mu$ measures $X$, and $\mu(Y)=\mu(Y a)$ for $Y \subseteq X^{-1} X$ and $a \in X^{-1} X$. To avoid too technical a language we will state the results using the Ind-definable group $\widetilde{G}=\bigcup_{n}\left(X^{-1} X\right)^{n}$, indicating occasionally how to restrict to $\left(X^{-1} X\right)^{3}$. The reader is welcome to ignore these refinements at a first reading.

An $\bigwedge$-definable subset of $\left(X^{-1} X\right)^{3}$ closed under $m$ and ${ }^{-1}$ will be called an $\bigwedge$-definable subgroup of $\widetilde{G}$ (though in the local setting there is a priori no group of which it is a substructure). The main case is that of countable intersections; in this case one can write $H=\bigcap_{n \in \mathbb{N}} H_{n}$, with $H_{n}$ definable, $H_{n}=H_{n}^{-1}$, and $H_{n} H_{n} \subseteq H_{n+1}$. It is easy to see that any $\bigwedge$-definable subgroup is an intersection of such countably- $\bigwedge$-definable subgroups. $\widetilde{G} / H$ is bounded if for any definable subset $Y$ of $\widetilde{G}$, the $\bigwedge$-definable equivalence relation: $y^{-1} y^{\prime} \in H$ has boundedly many classes in the sense of $\$ 1.5$. (Equivalently, if $\widetilde{G}, H$ are defined over $M_{0}$, the cardinality of $\widetilde{G}(N) / H(N)$ remains bounded when $N$ runs over all elementary extensions of $M_{0}$.)

Let $\widetilde{G}$ be generated by the definable set $X$. Let $\mu$ be an ideal on $\widetilde{G}$, invariant under right translations by elements of $X$ (i.e. $Z \in \mu$ iff $Z b \in \mu, b \in X$ ); equivalently, $\mu$ is invariant under right translations by elements of $\widetilde{G}$. Assume $\mu(X)>0$. Recall that a partial type $Q$ is called wide if $\mu\left(Q^{\prime}\right)>0$ for any definable $Q^{\prime} \supseteq Q$.

A definable subset $Z$ of $\widetilde{G}$ is called right generic if finitely many right translates of $Z$ cover any given definable subset of $\widetilde{G}$. If $Z$ is right generic, then clearly $\mu(Z)>0$. In the converse direction we have the observation, due to Ruzsa in the combinatorics literature, and Newelski in the model theory literature, that if $\mu(Z)>0$, then $Z^{-1} Z$ is right generic. We state this as a lemma for later reference.

Lemma 3.2. Let $\mu$ be an ideal on $\widetilde{G}=\langle X\rangle$, invariant under right translations by elements of $X$, and with $\mu(X)>0$. If $Q$ is a wide partial type, then so is $Q^{-1} Q$. If $Z$ is a definable set with $\mu(Z)>0$, then $Z^{-1} Z$ is right generic.

Proof. The statement for partial types follows by definition from the same statement for definable sets; so consider a definable set $Z$ with $\mu(Z)>0$. We have to show that $Z^{-1} Z$ is right generic, and wide.

Let $X_{n}=\left(X X^{-1}\right)^{n}$; say $Z \subseteq\left(X X^{-1}\right)^{n}$, and let $\left\{Z a_{i}: i \in I\right\}$ be a maximal collection of pairwise disjoint subsets of $Z$, with $a_{i} \in X_{n}$. We claim that $I$ is finite. Otherwise, by the usual Ramsey/compactness argument on existence of indiscernibles, one can find indiscernible $\left(a_{i}: i \in \mathbb{N}\right)$ with $a_{i} \in X_{n}$ and $Z a_{m} \cap$ $Z a_{m^{\prime}}=\emptyset$ for $m \neq m^{\prime}$; by the S1 property, since $\mu\left(Z a_{i}\right)>0$ for each $i$ by right invariance, while $\mu\left(Z a_{i} \cap Z a_{j}\right)=0$ for $i \neq j, I$ must be finite. If $a \in X_{n}$, then $Z a \cap Z a_{i} \neq \emptyset$ for some $i$; so $a \in Z^{-1} Z a_{i}$. This shows that $Z^{-1} Z$ is right-generic.

In particular, $X \subseteq \bigcup_{b \in B} X^{-1} X_{b}$, for some finite $B$; since $\mu(X)>0$ it follows that $\mu\left(X^{-1} X b\right)>0$ for some $b \in B$, so $\mu\left(X^{-1} X\right)>0$. 
In the local case, we say that $Z \subseteq\left(X^{-1} X\right)^{2}$ is right-generic if finitely many translates $Z b(b \in X)$ cover $X^{-1} X$. Again if $Z \subseteq X^{-1} X$ has positive $I$-measure, then $Z^{-1} Z$ is right-generic.

Lemma 3.3 (cf. [26]). Let $H$ be an $\bigwedge$-definable subgroup of $\widetilde{G}$. Then $\widetilde{G} / H$ is bounded iff every definable set containing $H$ is right-generic. For any right invariant S1-ideal $\mu$ on $\widetilde{G}$ this is also equivalent to: $H$ is wide.

Proof. Consider $H=\bigcap H_{n}$ as above. If each $H_{n}$ is generic, since $\widetilde{G}$ is a countable union of definable sets, there exists a countable set $C_{n}$ such that $H_{n} C_{n}=\widetilde{G}$. Let $C=\bigcup_{n} C_{n}$. Let $\pi: \widetilde{G} \rightarrow \widetilde{G} / H$ be the natural map. We say that a sequence $u_{n}$ of elements of $C$ converges to $u H \in \widetilde{G} / H$ if for each $m$, for all sufficiently large $n$, we have $H_{m} u_{n}=H_{m} u$. Then each sequence has at most one limit, and each point of $\widetilde{G} / H$ is the limit of some sequence from $C$. Hence the cardinality of $\widetilde{G} / H$ is at most continuum. (We will later define the "logic topology" on $\widetilde{G} / H$; in this language we have just shown that it is separable.)

Conversely if $\widetilde{G} / H$ is bounded, let $X$ be a definable subset of $\widetilde{G}$. The condition: $H_{k+1} x \cap H_{k+1} y=\emptyset$ is a definable relation on $(x, y)$, since $H_{k+1}$ is definable. Say $\widetilde{G} / H$ is bounded by $\lambda$; then a fortiori there cannot be more than $\lambda$ distinct $\left(a_{i}\right)$ with $H_{k+1} a_{i}$ disjoint. Compactness applies, so any such family is finite. Let $\left(a_{i}\right)$ be a maximal family $H_{k+1} a_{i}$ of disjoint cosets of $H_{k+1}$, with $a_{i} \in X$. Then there are finitely many elements $a_{i}$ in the family, and it follows that $X \subseteq \bigcup_{i} H_{k+1}^{-1} H_{k+1}^{-1} a_{i}=$ $\bigcup_{i} H_{k} a_{i}$, i.e. $H_{k}$ is right-generic.

Given a right invariant S1-ideal $\mu$, if $H$ is wide, then there can be no infinite family of disjoint cosets of $H_{k+1}$, so as above $H_{k}$ is generic. Conversely if $H_{k}$ is generic, then $\mu\left(\bigcup_{j} H_{k} b_{j}\right)>0$ for some finite set $b_{1}, \ldots, b_{l}$, so $\mu\left(H_{k} b_{j}\right)>0$, and by right invariance $\mu\left(H_{k}\right)>0$.

If an $\Lambda$-A-definable subgroup of bounded index exists, then there is a minimal one; it is denoted $\widetilde{G}_{A}^{00}$. For a discussion of the dependence on $A$, see [25].

Lemma 3.4. $\widetilde{G}_{A}^{00}$ is normal in $\widetilde{G}$.

Proof. Let $H=\widetilde{G}_{A}^{00}$. Then $H$ has boundedly many $\widetilde{G}$-conjugates; their intersection is an $\Lambda$-definable normal subgroup $N$ of $\widetilde{G}$. On the face of it the definition of $N$ requires additional parameters, but $N$ is $\operatorname{Aut}(\mathbb{U} / A)$-invariant, and in general if an $\bigwedge$-definable set is invariant under $\operatorname{Aut}(\mathbb{U} / A)$, then it is an infinite intersection of $A$-definable sets.

Theorem 3.5. Let $M$ be a model, $\mu$ an $M$-invariant $S 1$-ideal on definable subsets of $\widetilde{G}$, invariant under (left or right) translations by elements of $\widetilde{G}$. Let $q$ be a wide type over $M$ (contained in $\widetilde{G}$ ). Assume:

$(F)$ There exist two realizations $a, b$ of $q$ such that $t p(b / M a)$ does not fork over $M$ and $t p(a / M b)$ does not fork over $M$.

Then there exists a wide, $\Lambda$-definable over $M$ subgroup $S$ of $G$. We have $S=$ $\left(q^{-1} q\right)^{2}$; the set $q q^{-1} q$ is a coset of $S$. Moreover, $S$ is normal in $\widetilde{G}$, and $S \backslash q^{-1} q$ is contained in a union of nonwide $M$-definable sets.

Some remarks before turning to the proof. 
(1) It follows from the statement of the theorem that $S$ can have no proper $M$ - $\bigwedge$-definable subgroups of bounded index. For suppose such a subgroup $T$ exists. Then $q$ is contained in a bounded union of cosets of $T$. Being a complete type over a model, it is contained in a single coset. But then $q^{-1} q q^{-1}$, a coset of $S$, is contained in a coset of $T$; so $S=T$.

(2) The statement about $S \backslash q^{-1} q$ can be read to say that a random element of $S$ lies in $q^{-1} q$; for instance when $M$ is countable, and $\mu$ is the ideal of definable measure zero sets for some finitely additive measure $\mu$ on the Boolean algebra of $M$-definable sets, $\mu$ extends to a Borel measure on the space of types, and almost all types of elements of $S$ lie in $q^{-1} q$.

(3) When $\mu$ is the zero-ideal of a measure, note that translation invariance is assumed of the ideal, not of the measure. In particular, regardless of unimodularity, this assumption is true for Haar measures on a locally compact group.

(4) (Weakening of left invariance). Most of the proof is devoted to showing that $S=\left(q^{-1} q\right)^{2}$ is a subgroup of $\widetilde{G}$, and $q q^{-1} q$ is a coset of $S$. For this, left-translation invariance can be replaced with existence of an $f$-generic extension of $q$, in the sense of [26, i.e. the existence of an $M$-invariant ideal $J$ containing the forking ideal, and with $q$ wide for $J$. We will use such a $J$ in Claims $3^{\prime}$ and $5^{\prime}$ (without assuming that $\mu=J$ ). The statement is essentially that left generics do not fork, and involves $\mu$ but not $J$.

The word "wide" will refer to $\mu$ unless explicitly qualified.

Normality of $S$ will also follow under these assumptions, but we do not obtain the final statement about $S \backslash q^{-1} q$ in this case.

(5) In place of any form of left translation invariance, we could use a stronger Fubini-type assumption on $\mu$ itself. (In Claim $3^{\prime}$ of the theorem, we need to find $\left(c_{1}, c_{2}, a\right)$ with $t p\left(c_{i} / M\right)$ specified, $c_{i} \in q^{-1} q$, and with $\operatorname{tp}\left(a / M\left(c_{1}, c_{2}\right)\right)$ wide. Given a version of Fubini we can achieve this by choosing $a$ first, then $c_{1}, c_{2}$.)

(6) (Locality). Inspection of the proof will show that for all assertions except the normality of $S$, we only use $\mu$ (as an S1 ideal) on definable subsets of $X X^{-1} X$. To show normality of $S$, we also require $X a X^{-1}$, where $a \in X$ or $a \in X^{-1}$. Moreover the group structure is used only up to $\left(X^{-1} X\right)^{3}$. This is explicitly so everywhere except in Claim 5 . There, note that $q c \subseteq X X^{-1} X$. Hence $q c \cap Y \subseteq X X^{-1} X$ for any set $Y$, and it makes sense to say that this intersection is wide. In the proof, by the time we use $q a b_{1}$, we know that $a b_{1}$ is in $q^{-1} q$.

It is also possible to combine (4) and (6); see Example 3.8

(7) The theorem implies that $S \subseteq X^{-1} X X^{-1} X$, or that for an appropriate translate $Y=a^{-1} X$, we have $S=Y Y^{-1} Y$. Example 6.1.10 of [8] shows that this cannot be improved to $S \subseteq X^{-1} X$.

(8) An easy Löwenheim-Skolem argument shows that the theorem reduces to the case where the language is countable, and $M$ is countable.

(9) We show in fact that $S \backslash S t_{0}(q)$ is contained in a union of nonwide $M$ definable sets, where $S t_{0}(q)=\{s: q s \cap q$ is wide $\}$. If $s \in S$ is arbitrary now, and $t p\left(s^{\prime} / M(s)\right)$ is wide, then $t p\left(s^{\prime} s / M\right)$ is wide, so $s^{\prime}, s^{\prime} s \in S t_{0}(q)$. Hence $s=\left(s^{\prime}\right)^{-1}\left(s^{\prime} s\right) \in S t_{0}(q)^{-1} S t_{0}(q)=S t_{0}(q)^{2}$. 
(10) The assumption that $M$ is a model, rather than just a substructure of the universal domain, is used via the consequence that any type extends to an invariant type; thus Theorem 2.18 applies to any type $t p(a)$. See Remark 2.19

(11) The proof uses both the nonforking ideal and the ideal of wide sets with respect to $\mu$ (or $J$ ). The former allows Theorem 2.18 to be used for an arbitrary type, since any type has a nonforking extension. On the other hand the ideal of wide sets, unlike the nonforking ideal, enjoys translation invariance.

Proof of Theorem 3.5. We also write $q$ to denote $\{a: t p(a / M)=q\}$, and $q^{-1}=$ $\left\{a^{-1}: t p(a / M)=q\right\}$.

Given two subsets $X, Y$ of $\widetilde{G}$, let

$$
X \times{ }_{n f} Y=\{(a, b) \in X \times Y: t p(b / M(a)) \text { does not fork over } M\} .
$$

Let $Q=\left\{a^{-1} b:(a, b) \in q \times_{n f} q\right\}$. Let $J$ be as in Remark (4) (or just set $J=\mu$ for the basic statement of the theorem), and set $Q^{\prime}=\left\{a^{-1} b: a, b \in\right.$ $q, t p(b / M a)$ is $J$-wide .

Note that $q q^{-1}$ is obviously wide by right-invariance, and similarly $q^{-1} q$ is wide assuming left-invariance. If we wish to avoid the left-invariance assumption, but are willing to use $\mu$ on $X^{2}$ instead, then wideness of $q^{-1} q$ follows from Lemma 3.2.

Throughout this proof, we will use the fact (Lemma 2.10) that wideness of $q x \cap$ $q y^{-1}$ is a stable relation between $x$ and $y$. By Lemma 2.3, or Theorem 2.18, for any two types $p_{1}, p_{2}$, this relation holds for one pair $\left(a_{1}, a_{2}\right) \in p_{1} \times_{n f} p_{2}$ iff it holds for all pairs iff it holds for one or all pairs $\left(a_{2}, a_{1}\right)$ in $p_{2} \times_{n f} p_{1}$.

Claim 1. $q^{-1} q \subseteq Q Q$.

Proof. Let $a, b \in q$. Using $(\mathrm{F})$, find $c \models q$ such that $t p(a / M c)$ does not fork over $M$, and $t p(c / M a)$ does not fork over $M$. By extending $t p(c / M a)$ to a type over $M(a, b)$ and realizing this type, we may assume that $t p(c / M a b)$ does not fork over $M$. So we have $(b, c) \in q \times_{n f} q$, and $(c, a) \in q \times_{n f} q$. So $b^{-1} c, c^{-1} a \in Q$; hence $b^{-1} a \in Q Q$.

Claim 2. For all $(a, b) \in q \times_{n f} q, q a^{-1} \cap q b^{-1}$ is wide.

Proof. By Theorem 2.18, it suffices to show that for some $(a, b) \in q \times_{n f} q, q a^{-1} \cap q b^{-1}$ is wide. Let $a_{1}, a_{2}, \ldots$ be an $M$ - indiscernible sequence of elements of $q$, such that $\operatorname{tp}\left(a_{i} / A \cup\left\{a_{j}: j<i\right\}\right)$ does not fork over $M$. Then $\left(a_{i}, a_{j}\right) \in q \times_{n f} q$ for any $i<j$. It suffices to show that $q a_{1}^{-1} \cap q a_{2}^{-1}$ is wide; by compactness, for any definable set $D$ containing $q$, it suffices to show that $\mu\left(D a_{1}^{-1} \cap D a_{2}^{-1}\right)>0$. This is clear since $\mu$ is an S1-ideal, and by right-invariance, $\mu\left(D a_{i}^{-1}\right)>0$.

Claim $3^{\prime}$. For all $\left(c_{1}, c_{2}\right) \in\left(q^{-1} q\right) \times_{n f} Q^{\prime}, q c_{1}^{-1} \cap q c_{2}^{-1}$ is wide.

Proof. Let $p_{i}=t p\left(c_{i} / M\right)$. As in Claim 2, it suffices to see that $q c_{1}^{-1} \cap q c_{2}^{-1}$ is wide for some $\left(c_{1}, c_{2}\right) \in p_{1} \times_{n f} p_{2}$. Let $a_{0} \models q$. Then there exists $a_{1} \in q$ with $t p\left(a_{0}^{-1} a_{1} / M\right)=p_{1}$. Since $c_{2} \in Q^{\prime}$, there exists $a_{2}^{\prime}$ such that $r=t p\left(a_{2}^{\prime} / M\left(a_{0}\right)\right)$ is $J$-wide and $t p\left(a_{0}^{-1} a_{2}^{\prime} / M\right)=p_{2}$; extend $r$ to a $J$-wide type $r^{\prime}$ over $M\left(a_{0}, a_{1}\right)$, and let $a_{2} \models r^{\prime}$. We thus have $\left(a_{0}, a_{1}, a_{2}\right) \in(q \times q) \times_{n f} q$, with $t p\left(a_{0}^{-1} a_{i} / M\right)=p_{i}$ for $i=1,2$. Note also, using left invariance of $J$, that $\operatorname{tp}\left(a_{0}^{-1} a_{2} / M\left(a_{0}, a_{1}\right)\right)$ is $J$-wide, hence so is $t p\left(a_{0}^{-1} a_{2} / M\left(a_{0}^{-1} a_{1}\right)\right)$, so it does not fork over $M$. 
By Claim 2 we have that $q a_{1}^{-1} \cap q a_{2}^{-1}$ is wide. By the right-invariance of $\mu$, $q a_{1}^{-1} a_{0} \cap q a_{2}^{-1} a_{0}$ is wide.

Claim 3. For all $(c, d) \in\left(q^{-1} q\right) \times_{n f} Q, q c^{-1} \cap q d^{-1}$ is wide.

Proof. Let $d=a^{-1} b$, with $t p(b / M(a))$ wide for the forking ideal over $M$. We have to show that $q c^{-1} \cap q b^{-1} a$ is wide. By Theorem 2.18, it suffices to show this for one instance $(c, b, a)$ with $t p(b, a)$ specified and such that $t p(b, a / M(c))$ does not divide over $M$. We may thus take $t p(a / M(c))$ to be a nonforking extension of $q=t p(a / M)$, and $t p(b / M(a, c))$ to be a nonforking over $M$ extension of $t p(b / M(a))$. The latter is possible using the assumption that $t p(b / M(a))$ does not fork over $M$.

By right-invariance, we need to show that $q c^{-1} a^{-1} \cap q b^{-1}$ is wide. We apply Theorem 2.18 to the pair $(a, b)$ (viewed as a single tuple) and $c$. So it suffices to show that $q c^{-1} a^{-1} \cap q\left(b^{\prime}\right)^{-1}$ is wide, where $t p(b / M)=\operatorname{tp}\left(b^{\prime} / M\right)$ and $t p\left(b^{\prime} / M(a, c)\right)$ is $J$-wide. By left-invariance of $J$, the type $\operatorname{tp}\left(a^{-1} b^{\prime} / M(a, c)\right)$ is $J$-wide, and hence $t p\left(a^{-1} b^{\prime} / M(c)\right)$ is $J$-wide; so $t p\left(a^{-1} b^{\prime} / M(c)\right)$ does not fork over $M$. Also $\operatorname{tp}\left(b^{\prime} / M(a)\right)$ is $J$-wide, so $a^{-1} b^{\prime} \in Q^{\prime}$. By Claim $3^{\prime}, q c^{-1} \cap q\left(a^{-1} b^{\prime}\right)^{-1}$ is wide. By right-invariance, $q c^{-1} a^{-1} \cap q\left(b^{\prime}\right)^{-1}$ is wide, as required.

Claim 4. Let $(b, a) \in Q \times_{n f} q^{-1} q$. Then $a b \in q^{-1} q$. In fact $q a \cap q b^{-1}$ is wide.

Proof. We have $a^{-1} \in q^{-1} q$. Since $M$ is a model, $t p\left(a^{-1} / M\right)$ extends to a global type $r$ finitely satisfiable type in $M$; so $r$ is $M$-invariant. Use Lemma 2.3 (1) and Claim 3 to conclude that $q c^{-1} \cap q b^{-1}$ is wide if $c=r \mid M(b)$. Now $t p(c / M(b))$ does not divide over $M$, so by Theorem 2.18, since $t p\left(a^{-1} / M(b)\right)$ does not divide over $M$ either, $q a \cap q b^{-1}$ is wide. In particular, for some $d, e \in q$ we have $d a=e b^{-1}$. So $a b=d^{-1} e \in q^{-1} q$.

Claim 5. Let $a \in q^{-1} q, b_{1}, \ldots, b_{n} \in Q$ and assume $t p\left(a / M\left(b_{1}, \ldots, b_{n}\right)\right)$ is wide. Then $a b_{1} \cdots b_{n} \in q^{-1} q$. In fact $q a \cap q\left(b_{1} \cdots b_{n}\right)^{-1}$ is wide.

Proof. Since $t p\left(a / M b_{1}\right)$ is wide, it does not fork over $M$ (Lemma 2.9). Hence by Claim 4 we have $a b_{1} \in q^{-1} q$. By right-invariance of $\mu, t p\left(a b_{1} / M\left(b_{1}, \ldots, b_{n}\right)\right)$ is wide, and in particular $t p\left(a b_{1} / M\left(b_{2}, \ldots, b_{n}\right)\right)$ is wide. By induction, $q a b_{1} \cap q\left(b_{2} \cdots b_{n}\right)^{-1}$ is wide. Multiplying by $b_{1}^{-1}$ on the right, $q a \cap q\left(b_{1} b_{2} \cdots b_{n}\right)^{-1}$ is wide. Hence as in Claim $4, a b_{1} \cdots b_{n} \in q^{-1} q$.

In view of Theorem 2.18, Claim 5 is also valid assuming $t p(a / M)$ is wide, and $\operatorname{tp}\left(a / M\left(b_{1}, \ldots, b_{n}\right)\right)$ does not fork over $M$. To show that $q q^{-1} q$ is a coset, we will later need a variant of Claim 5 , proved in the same way:

Claim $5^{\prime}$. Let $a \in q^{-1} q, b_{1}, \ldots, b_{n} \in Q$ and assume $t p\left(a^{-1} / M\left(b_{1}, \ldots, b_{n}\right)\right)$ is $J$-wide. Then $a b_{1} \cdots b_{n} \in q^{-1} q$. In fact $q a \cap q\left(b_{1} \cdots b_{n}\right)^{-1}$ is wide.

Proof. Since $t p\left(a^{-1} / M b_{1}\right)$ is $J$-wide, it does not fork over $M$, and so $t p\left(a / M b_{1}\right)$ does not fork over $M$. Hence by Claim 4 we have $a b_{1} \in q^{-1} q$. By left-invariance of $J, \operatorname{tp}\left(\left(a b_{1}\right)^{-1} / M\left(b_{1}, \ldots, b_{n}\right)\right)$ is $J$-wide, and in particular $\operatorname{tp}\left(\left(a b_{1}\right)^{-1} / M\left(b_{2}, \ldots, b_{n}\right)\right)$ is $J$-wide. By induction, $q a b_{1} \cap q\left(b_{2} \cdots b_{n}\right)^{-1}$ is wide. Multiplying by $b_{1}^{-1}$ on the right, $q a \cap q\left(b_{1} b_{2} \cdots b_{n}\right)^{-1}$ is wide. Hence as in Claim $4, a b_{1} \cdots b_{n} \in q^{-1} q$.

Claim 6. $Q^{n} \subset q^{-1} q q^{-1} q$.

Proof. Let $b_{1}, \ldots, b_{n} \in Q$. Let $a \in q^{-1} q$ with $t p\left(a / M\left(b_{1}, \ldots, b_{n}\right)\right)$ wide. Then $a b_{1} \cdots b_{n} \in q^{-1} q$, so $b_{1} \cdots b_{n}=a^{-1}\left(a b_{1} \cdots b_{n}\right) \in q^{-1} q q^{-1} q$. 
It follows from Claim 1 that $Q$ and $q^{-1} q$ generate the same subsemigroup, which is hence a group $S$. By Claim 6 , this group is in fact equal to the $\wedge$-definable set $q^{-1} q q^{-1} q$.

Since $q^{-1} q \subseteq S$, we have $q \subseteq b S$ for any $b \in q$, and so $q q^{-1} q \subseteq b S$. Conversely, choose $b \in q$. Any element $x$ of $b S$ can be written $x=b a_{1} \cdots a_{4}$ with $a_{i} \in Q$. Let $d \in q$ be such that $\operatorname{tp}\left(d / M\left(a_{1}, \ldots, a_{4}, b\right)\right)$ is $J$-wide. Let $e=d^{-1} b$. Then $\operatorname{tp}\left(e^{-1} / M\left(a_{1}, \cdots, a_{4}, b\right)\right)$ and hence $t p\left(e^{-1} / M\left(a_{1}, \cdots, a_{4}\right)\right)$ are $J$-wide. By Claim $5^{\prime}$ we have $e a_{1} \cdots a_{4} \in q^{-1} q$. So $x=b a_{1} \cdots a_{4} \in d q^{-1} q \subset q q^{-1} q$. Thus $q q^{-1} q=b S$.

We know that $S$ is an $\bigwedge$-definable group over $M$. I claim any $\bigwedge$-definable over $M$ subgroup of $S$ of bounded index must be equal to $S$. For let $T$ be such a subgroup. We have $q^{-1} q \subseteq S$, so $q \subseteq a S$ for any $a \in q$. Thus $q$ is contained in a left translate $R$ of $S$; we have $R=q S$, so $R$ is defined over $M$. Now $T$ acts on $R$ on the right; the equivalence relation induced is $\bigwedge$-definable over $M$ with boundedly many orbits. Since $q$ is a complete type over $M$, it has an $\operatorname{Aut}(\mathbb{U} / M)$-invariant extension to $\mathbb{U}$; this extension must pick a specific $T$-orbit $c T$, which is hence $\bigwedge$-definable over $M$; by completeness again, as the realizations of $q$ intersect $c T, q$ is contained in $c T$. But then $q^{-1} q \subseteq T$; so $S \subseteq T$.

We know at this point that $S$ has no proper $\bigwedge$-definable over $M$ subgroups of bounded index. Let $r$ be a type of elements of $X \cup X^{-1}$ over $M$. There cannot exist an unbounded family of cosets $a_{i} S$ with $a_{i} \in r$, for then the sets $a_{i} b q$ would also be disjoint for any $b \in q^{-1}$, so for some definable $X^{\prime}$ with $q \subset X^{\prime} \subset X$ the sets $a_{i} b X^{\prime}$ can be taken to be disjoint, contradicting the S1 property for $\mu$ within $r b X \subseteq\left(X \cup X^{-1}\right)^{3}$. Thus $r$ is contained in boundedly many left cosets of $S$, hence (being a complete type over a model) in one; call it $C_{r}$. So $C_{r}$ is $M$-definable, and hence the conjugate group $S^{r}=C_{r}^{-1} S C_{r}$ is $M$-definable.

For any $c \in X \cup X^{-1} \cup\{1\}, r=t p(c)$, the image of $q c$ in $G / S$ is bounded. Otherwise there is a large collection of disjoint sets of the form $a_{i} c S$, with $a_{i} \in q$. Pick $b_{0} \in q$; then $q^{-1} b_{0} \subseteq S$. The sets $a_{i} c S b_{0}^{-1}$ are also disjoint, hence so are the $a_{i} c q^{-1}$. Thus there exists a definable $X^{\prime} \subset X$ with $a_{i} c\left(X^{\prime}\right)^{-1}$ disjoint. So the sets $X c^{-1} a_{i}^{-1}$ are disjoint, and wide. But this contradicts the S1 property within $X c X^{-1}$. Thus $q c / S$ is bounded. It follows that $q$ is contained in boundedly many cosets of $c S c^{-1}=S^{r}$. So $q$ is contained in a single coset $g S^{r}$. It follows that $q^{-1} q \subseteq S^{r}$, so $S \subseteq S^{r}$. Similarly $S \subseteq S^{r^{-1}}$, so $S^{r} \subseteq S$ and $S^{r}=S$. This shows that $X \cup X^{-1}$ normalizes $S$; i.e., $S$ is normal in $\widetilde{G}$.

At this point we begin using left-invariance freely.

We argued above that $q^{-1} q$ is wide; in particular, $S$ is wide. $Q$ is also wide: suppose otherwise. So $Q \subseteq D$ for some definable $D$ with $\mu(D)=0$. Let $a \in q$. Then $a^{-1} q$ is wide. So $a^{-1} q \backslash D$ is wide. However $q \backslash a D$ forks over $M$, since if $b \in q \backslash a D$, then $a^{-1} b \notin Q$, so $t p(b / M(a))$ forks over $M$. Thus $D^{\prime} \backslash a D$ lies in the forking ideal, for some definable $D^{\prime}$ containing $q$. By Lemma 2.9 we have $\mu\left(D^{\prime} \backslash a D\right)=0$; so $\mu\left(a^{-1} D^{\prime} \backslash D\right)=0$. It follows that $\mu\left(a^{-1} D^{\prime}\right)=0$ and $\mu\left(D^{\prime}\right)=0$, contradicting the wideness of $q$.

We finally show that $S$ is contained in $q^{-1} q$ up to a union of nonwide definable sets. Let $r$ be a wide type over $M$ extending $S$; we have to show that $r \subseteq q^{-1} q$. Pick $a_{0} \in r$ and $c \in Q$ with $t p\left(c / M\left(a_{0}\right)\right)$ wide. As $a_{0}^{-1} \in S$, we may write $a_{0}=b_{1} \cdots b_{n}$ with $b_{i} \in Q$; also as $c \in Q$ we have $c \in q^{-1} q$. Thus Claim 5 applies (with $c$ playing the role of $a$ of Claim 5), and we obtain that $q c \cap q a_{0}$ is wide. Choose $b_{0} \in r$ with $t p\left(b_{0} / M(c)\right)$ wide. In particular $t p\left(b_{0} / M(c)\right)$ does not fork over $M$. By stability 
of the relation and Lemma 2.3 (3), $q c \cap q b_{0}$ is wide too. Thus $b_{0} c^{-1} \in q^{-1} q$. Now $t p\left(b_{0} c^{-1} / M(c)\right)$ is a right translate of $t p\left(b_{0} / M(c)\right)$, so it is wide. By Claim 5 (or 3), $b_{0}=\left(b_{0} c^{-1}\right) c \in q^{-1} q$ (and $q b_{0} c^{-1} \cap q c^{-1}$ is wide; so $q b_{0} \cap q$ is wide). So $r \subseteq q^{-1} q$, as required. In fact this shows that $r \subseteq S t_{0}(q)$, in the notation of Remark (9).

Corollary 3.6. Let $\mu$ be an invariant $S 1$-ideal on definable subsets of $\widetilde{G}$, invariant under translations by elements of $\widetilde{G}$. Then there exists a model $M$ and a wide, $\Lambda$ definable over $M$ subgroup $S$ of $G$, with $\widetilde{G} / S$ bounded. For an appropriate complete type $q$ over $M$ we have $S=\left(q^{-1} q\right)^{2}$, and the complement $S \backslash q^{-1} q$ is contained in a union of nonwide $M$-definable sets.

If $\mu$ satisfies the conditions of Lemma 2.17 over a model $M_{0}$, or if $\mu$ is $\bigvee$ definable over $M_{0}$, then one can take $M=M_{0}$.

Proof. Lemma 2.16 provides $M$ and an $M$-invariant global type $q^{*}$ such that if $q=q^{*}|M, a \models q| M$ and $b \models q^{*} \mid M(a)$, then $t p(a / M(b))$ is $\mu$-wide. This implies (F). In case the assumptions of Lemma 2.17 or Lemma 2.15 hold, these lemmas provide a type over $M_{0}$ with (F) and so Theorem 3.5 applies with $M=M_{0}$.

Example 3.7. Consider the theory of divisible ordered Abelian groups $(G,+,<)$, or any o-minimal expansion, and let $M$ be a model. We have a two-valued definable measure $\mu$, assigning measure 0 to any bounded definable set. A two-valued invariant measure is always $\mathrm{S} 1$. The measure $\mu$ is translation invariant. Let $q_{A}$ be the set of all measure-one $M$-definable formulas over $A, q=q_{M}$. If $a \models q_{M}$ and $b \models q_{M(a)}$, then $\operatorname{tp}(a / M b)$ does not fork over $M$ since it is finitely satisfiable in $M$, and $t p(b / M a)$ does not fork over $M$ since it extends to an $M$-invariant type. Hence (F) of Theorem 3.5 holds. We can take $\widetilde{G}=G, X=\{x \in G: x>0\}$. The subgroup $S$ is then $G$. Note that $q^{-1}$ is not wide in this example.

Here is an example of the situation discussed in comments (4), (6), where the $\bigwedge$-definable group $S$ is not normal.

Example 3.8. Consider the theory ACVF of algebraically closed valued fields, say of residue characteristic 0 ; the field of Puiseux series over $\mathbb{C}$ is a model. Alternatively, let $M$ be an ultraproduct of the $p$-adic fields $\mathbb{Q}_{p}$. Let $K$ denote the valued field, $\mathcal{O}$ the valuation ring, $\mathcal{M}$ the maximal ideal. Let $G$ be the semi-direct product of the additive group $G_{a}$ with the multiplicative group $G_{m}$. So $G=T U$, where $T, U$ are Abelian subgroups, $U=G_{a}$ normal, $T \cong G_{m}$. Let $t \in K$ be an element of valuation $>0$, and let $g$ be the corresponding element of $G$, so that conjugation by $g$ acts on $U$ as multiplication by $t$. Let $U_{0}=\left\{x \in K: \bigvee_{m \in \mathbb{N}} \operatorname{val}(x) \geq-m \operatorname{val}(g)\right\}$. View $\mathcal{O} \leq U_{0}$ as subgroups of $U$. Within $G$, let $X=g \mathcal{O}$. The group $\widetilde{G}$ generated by $X$ is $g^{\mathbb{Z}} U_{0}$. Let $p$ be a generic type of $\mathcal{O}$; it avoids any coset of $\mathcal{M}$ in $\mathcal{O}$. Let $\mu$ be the right-invariant ideal generated by $g \mathcal{M}$, and $J$ the left-invariant ideal generated by $g \mathcal{M}$. These are not the same; notably $\mathcal{O}$ is in $J$ but not in $\mu . \mu$ is not $\mathrm{S} 1$, but it is so when restricted to $X=X X^{-1} X$. Let $q=g p$. As in Remarks (4) and (6), the proof of Theorem 3.5 goes through to give a subgroup $S$, namely $\mathcal{O}$ (it is definable in this case). But $\mathcal{O}$ is not a normal subgroup of $\widetilde{G}$.

Definition 3.9. We call $X$ a near-subgroup of $G$ if there exists an invariant S1ideal $\mu$ on definable subsets of $\left(X \cup X^{-1}\right)^{3}$, with $\mu(X)>0$, and with $\mu(Y)=\mu\left(Y^{\prime}\right)$ whenever $Y, Y^{\prime} \subseteq X X^{-1} X$ and $Y^{\prime}=c Y$ or $Y^{\prime}=Y c$ for some $c$. 
We will see in Corollary 3.11 that asking for $\mu$ defined on $\widetilde{G}=\cup_{n}\left(X \cup X^{-1}\right)^{n}$ would result in the same definition; in later sections we will work with this stronger definition.

Remark 3.10. Lou van den Dries has shown that a weaker condition suffices: $0 \in X$, and $\mu$ is defined on $X X^{-1} X$. Moreover the element $c$ (which must by definition be in $\left.\left(X \cup X^{-1}\right)^{6}\right)$ can in fact be chosen so that all products are taken within $X X^{-1} X$. This condition is essentially sharp, in view of Example 3.8. See [12.

When $X$ is finite, any right-invariant measure must be proportional to the counting measure. Asymptotically, when $(X, G)$ vary in some family, we have that every ultraproduct is a near-subgroup iff $\left|X X^{-1} X\right| /|X|$ is bounded in the family.

The following corollary of Theorem 3.5 is analogous to Lemma 3.4 of [50]; the point is that we do not assume a priori that $\left(X^{-1} X\right)^{n}$ has finite measure. The Fubini-type assumption on the ideal is much weaker here, but the conclusion is purely qualitative. We state the extension lemma for S1 ideals; a similar statement for measures is also valid, with a similar proof.

Corollary 3.11. Let $X$ be a near-subgroup of $G$. Then for any $n,\left(X^{-1} X\right)^{n}$ is contained in a finite union of right translates of $\left(X^{-1} X\right)^{2} . \mu$ extends to an invariant $S 1$-ideal $\mu^{\prime}$ on $\bigcup_{n}\left(X^{-1} X\right)^{n} ; \mu^{\prime}$ is the unique right-invariant ideal extending $\mu \mid\left(X^{-1} X\right)^{2}$.

Proof. For this we may add parameters and work over a model. Let $\widetilde{G}$ be the group generated by $X$. By Theorem 3.5 and Remark (6) to that theorem, there exists a wide $\bigwedge$-definable normal subgroup $S$ of $\widetilde{G}$. The proof also shows that $S \subseteq\left(X^{-1} X\right)^{2}$ and that the image of $X$ modulo $S$ has bounded cardinality. Hence $\widetilde{G} / S$ is bounded, and in particular for any $n,\left(X^{-1} X\right)^{n}$ is contained in boundedly many cosets of $S$, and hence in boundedly many right translates of $\left(X^{-1} X\right)^{2}$. By compactness, finitely many right cosets of $\left(X^{-1} X\right)^{2}$ suffice to cover $\left(X^{-1} X\right)^{n}$. If $D$ is a definable subset of $\bigcup_{n}\left(X^{-1} X\right)^{n}$, it follows that we can write $D=\bigcup_{i} D_{i} b_{i}$, where $D_{i} \subseteq\left(X^{-1} X\right)^{2}$ and $b_{i} \in\left(X^{-1} X\right)^{n+2}$.

Define $\mu^{\prime}$ to be the collection of all definable sets $\bigcup_{i} D_{i} b_{i}$, where $D_{i}$ is a definable subset of $\left(X^{-1} X\right)^{2}, b_{i} \in \bigcup_{n}\left(X^{-1} X\right)^{n}$, and $\mu\left(D_{i}\right)=0$. This is clearly a rightinvariant ideal whose restriction to a definable subset of $\left(X^{-1} X\right)^{3}$ is precisely $\mu$. (If $Y$ is a definable subset of $\left(X^{-1} X\right)^{3}$ and $Y=\bigcup_{i} D_{i} b_{i}$ as in the definition of $\mu^{\prime}$, then by invariance we have $\mu\left(D_{i} b_{i}\right)=0$ for each $i$, so $\mu(Y)=0$.)

If $\mu^{\prime \prime}$ is any right-invariant ideal $\mu^{\prime}$ extending $\mu$, and if $D$ is a definable subset of $\left(X^{-1} X\right)^{n}$, write $D=\bigcup_{i} D_{i} b_{i}$ where $D_{i} \subseteq\left(X^{-1} X\right)^{2}$ and $b_{i} \in\left(X^{-1} X\right)^{n+2}$. We have $\mu^{\prime \prime}(D)=0$ iff $\mu^{\prime \prime}\left(D_{i}\right)=0$ for each $i$ iff $\mu\left(D_{i}\right)=0$ for each $i$. This shows that $\mu^{\prime \prime}=\mu^{\prime}$.

To see that $\mu^{\prime}$ is $\mathrm{S} 1$, it suffices to show for each $n$ that the restriction to $\left(X^{-1} X\right)^{n}$ is S1. As above, write $\left(X^{-1} X\right)^{n}=\bigcup_{j=1}^{l} D_{j} b_{j}$. It is clear that any ideal on the union of finitely many sets must be S1 if the restriction to each of these sets is S1. So it suffices to show that $\mu^{\prime} \mid\left(D_{j} b_{j}\right)$ is $\mathrm{S} 1$ for each $j$. But $\mu^{\prime} \mid\left(D_{j} b_{j}\right)$ is isomorphic, via translation by $b_{j}$, to $\mu \mid D_{j}$, which is $\mathrm{S} 1$.

This kind of characterization incidentally makes some functorialities evident, that are not so directly from the definition of a near-subgroup or an approximate subgroup; see Remark 4.10 (0),(2). 
Given elements $a_{1}, \ldots, a_{l}$ and $b_{1}, \ldots, b_{m}$ of $G$, let $A_{i}=\left\{x^{-1} a_{i} x: x \in X\right\}$ be the set of $X$-conjugates of $a_{i}$, and let $W_{n}\left(a_{1}, \ldots, a_{l}, b_{1}, \ldots, b_{m}\right)$ be the set of words of length $\leq n$ in $A_{1} \cup \ldots \cup A_{l} \cup\left\{b_{1}, \ldots, b_{m}\right\}$. Let $d\left(X ; a_{1}, \ldots, a_{l}\right)$ be the smallest integer $n$ such that $X \subseteq W_{n}\left(a_{1}, \ldots, a_{l} ; b_{1}, \ldots, b_{l}\right)$ for some $b_{1}, \ldots, b_{l} \in X$, or $\infty$ if there is no such $n$.

Proposition 3.12. For any $k, l, n \in \mathbb{N}$, for some $M, K \in \mathbb{N}$, the following holds:

Let $G$ be a group, $X$ a finite subset. Assume $\left|X X^{-1} X\right| \leq k|X|$. Also assume that there exist $x_{1}, \ldots, x_{M} \in X$ such that:

(*) for any $1 \leq i_{0}<i_{1}<\cdots<i_{l} \leq M, d\left(X, x_{i_{0}}^{-1} x_{i_{1}}, \ldots, x_{i_{0}}^{-1} x_{i_{l}}\right) \leq n$.

Then there exists a subgroup $S$ of $G, S \subseteq\left(X^{-1} X\right)^{2}$, such that $X$ is contained in $\leq K$ cosets of $S$.

Proof. Fix $k, l, n$. Suppose there are no such $M, K$; then there are groups $G_{M}$ and $X=X_{M} \subset G_{M}$ such that there exist $x_{1}, \ldots, x_{M}$ with $(*)$, and there is no subgroup $S$ of $G, S \subseteq\left(X^{-1} X\right)^{2}$, such that $X$ is contained in $\leq M$ cosets of $S$. Consider $\left(G_{M}, X_{M}, \cdot\right)$ as a structure and enrich it using the $Q_{\alpha}$-quantifiers for the normalized counting measure on $X_{M}$, as in 2.6 . By compactness, there exists a countably saturated group $G$ and a subset $X$ such that there exists an infinite indiscernible sequence $x_{1}, x_{2}, \ldots \in X$ such that

(i) $\left(^{*}\right)$ holds for any $1 \leq i_{0}<i_{1}<\cdots<i_{l}<\infty$.

(ii) For any definable subgroup $S$ of $G$ with $S \subseteq\left(X^{-1} X\right)^{2}, X$ is not contained in finitely many right translates of $S$.

Let $\widetilde{G}$ be the subgroup of $G$ generated by $X$. By Theorem 3.5 there exists an $\bigwedge$-definable normal subgroup $S$ of bounded index in $\widetilde{G}$, with $S \subseteq\left(X^{-1} X\right)^{2}$. Since the sequence $x_{1}, x_{2}, \ldots$ is indiscernible and $G / S$ is bounded, all $x_{i}$ lie in the same coset of $S$. So the elements $y_{i}=x_{1}^{-1} x_{i}$ all lie in $S$. Now $d\left(X, y_{1}, \ldots, y_{l}\right) \leq n$; so $X \subseteq W_{n}\left(y_{1}, \ldots, y_{l} ; b_{1}, \ldots, b_{l}\right)$ for some $b_{1}, \ldots, b_{l} \in X$. Let $N$ be the normal subgroup of $\widetilde{G}$ generated by the $y_{i}$, and let $\mathbf{X}$ be the image of $X$ modulo $N$. Then $\mathbf{X} \subseteq W_{n}\left(1, \ldots, 1 ; \bar{b}_{1}, \ldots, \bar{b}_{l}\right)$, where $\bar{b}_{i}=b_{i} N$. Hence $\mathbf{X}$ is finite. As $S \subseteq\left(X^{-1} X\right)^{2}$, it follows that the image of $S$ modulo $N$ is finite, i.e. $[S: N]<\infty$. Since $N$ is $\bigvee$-definable, so is $S$. But $S$ is $\bigwedge$-definable; so it is a definable group. Now $N \subseteq S$, so $X$ is contained in finitely many translates of $S$, in contradiction to (ii).

By Ruzsa's argument, the condition $\left|a_{1}^{X} \cdots a_{l}^{X}\right| \geq|X| / m$ implies that $X \cup X^{2}$ is contained in the union of $\leq m$ translates $c_{j} a_{1}^{X} \cdots a_{l}^{X}\left(a_{l}^{-1}\right)^{X} \cdots\left(a_{1}^{-1}\right)^{X}$, with $c_{j} \in X$, so that $d\left(a_{1}, \ldots, a_{l} ; G\right) \leq \max (m, 2 l)$. We can now deduce Corollary 1.2 from Proposition 3.12 using Ramsey's theorem, but we will give a direct argument. We denote the $l$ th Cartesian power of $X$ by $X^{(l)}$.

Proof of Corollary 1.2, Fix $k, l, m$ and suppose for contradiction that the conclusion fails. Then for arbitrarily large $K$, letting $p=1-1 / K$, there exists a group $G_{K}$ and a finite subset $\left(X_{0}\right)_{K}, X=\left(X_{0}\right)_{K}^{-1}\left(X_{0}\right)_{K}$, such that the situation of Corollary 1.2 holds but no subgroup $S$ of $G_{K}$ with $S \subset X^{2}$ is contained in $\leq K$ cosets of $S$. Let $\mu_{K}$ be the counting measure on $G$, normalized so that $\mu_{K}(X)=1$, and let $\mu_{K}^{l}$ be the counting measure on $X^{l}$, similarly normalized. Let $Q_{K}$ be the set of $l$-tuples $\left(a_{1}, \ldots, a_{l}\right)$ such that $\mu_{K}\left(a_{1}^{X} \cdots a_{l}^{X} \mid\right) \geq 1 / m$; then $\mu_{K}^{l}\left(Q_{K}\right) \geq p=1-1 / K$.

By compactness (as in the proof of Proposition 3.12 and in 2.6 ) there exists a structure including a group $G$, a definable subset $X=X_{0}^{-1} X_{0} \subset G$, a definable measure $\mu$ on definable sets, with $1=\mu(X) \leq k \mu\left(X_{0}\right)<\infty$. There is also as well a 
definable measure $\mu^{l}$ on $l$-tuples, related to $\mu$ via Fubini's theorem; the only instance of Fubini we will need is the equality: $\mu^{l}\left(Y^{l}\right)=\mu(Y)^{l}$, holding for any definable $Y$. Further, there exists a definable set $Q \subseteq X^{(l)}$ such that if $\left(a_{1}, \ldots, a_{l}\right) \in Q$, then $\mu\left(a_{1}^{X} \cdots a_{l}^{X}\right) \geq 1 / m$, and $\mu^{l}(Q) \geq 1-1 / K$ for any $K=1,2, \ldots$, i.e. $\mu^{l}(Q)=1$. Finally, for no definable group $S \subseteq X X$ is $X$ contained in finitely many cosets of $S$.

We take $G$ to be countably saturated. Recall that countable saturation means that any countable family of definable sets with the finite intersection property has nonempty intersection; we will actually need it for the family $R_{j}$ below.

By Theorem 3.5 there exists an $\bigwedge$-definable normal subgroup $S$ of bounded index in $\widetilde{G}$. Find a countable set of definable (with parameters) equivalence relations $E^{j}$ on $X_{0}$, such that each $E^{j}$ has finitely many classes, $E^{j+1}$ refines $E^{j}$, and if $(a, b) \in E^{j}$ for each $j$, then $a^{-1} b \in S$. (For instance, say $S=\bigcap S_{j}$, and let $C^{j}$ be a maximal subset such that $S_{j} x \cap S_{j} y=\emptyset$ for $x \neq y \in C^{j}$; define $E^{j}$ so that $(x, y) \in E^{j}$ implies $\left\{c \in C^{j}: x S_{j} \cap c S_{j}=\emptyset\right\}=\left\{c \in C^{j}: y S_{j} \cap c S_{j}=\emptyset\right\}$. Alternatively note that if $a, b$ have the same type over some countable model, then $a^{-1} b \in S$.)

Some class $F_{j}$ of $E^{j}$ has measure $\epsilon_{j}>0$; so $\mu\left(F_{j}^{-1} F_{j}\right) \geq \epsilon_{j}>0$. Thus $\left(F_{j}^{-1} F_{j}\right)^{(l)}$ $\geq \epsilon_{j}^{l}$, and hence $\left(\operatorname{as} \mu(Q)=\mu\left(X^{l}\right)=1\right)$ we have $\mu\left(Q \cap\left(F_{j}^{-1} F_{j}\right)^{(l)}\right) \geq \epsilon_{j}^{l}>0$. Hence for each $j$ there exist $\left(a_{1}, \ldots, a_{l}\right) \in Q$ such that for each $i \leq l$, we have $a_{i}=b_{i}^{-1} c_{i}$ for some $\left(b_{i}, c_{i}\right) \in E^{j}$. As we took the $E_{j}$ to refine each other, this holds for any finite set of indices $j$ at once. In other words, the family of sets $\left\{R_{j}\right\}$ has the finite intersection property:

$$
R_{j}=\left\{\left(a_{1}, b_{1}, c_{1}, \ldots, a_{l}, b_{l}, c_{l}\right):\left(a_{1}, \ldots, a_{l}\right) \in Q, \bigwedge_{i \leq l}\left(a_{i}, b_{i}\right) \in E^{j}, \text { and } a_{i}=b_{i}^{-1} c_{i}\right\}
$$

By countable saturation, $\bigcap_{j} R_{j} \neq \emptyset$; i.e., there exist $\left(a_{1}, \ldots, a_{l}\right) \in Q$ and $b_{1}, c_{1}, \ldots$, $b_{l}, c_{l}$ such that for each $i \leq l$ we have $a_{i}=b_{i}^{-1} c_{i}$ and $\left(b_{i}, c_{i}\right) \in \bigcap_{j} E^{j}$. By the choice of $E^{j}$, this implies $a_{i} \in S$.

Now $S$ is normal in $\widetilde{G}$, so $a_{1}^{X} \cdots a_{l}^{X} \subseteq S$. Since $\mu\left(a_{1}^{X} \cdots a_{l}^{X}\right)>1 /(m+1)$, it follows that $S$ cannot have $\mu\left(X_{0} X\right)(m+1)$ disjoint cosets $x_{i} S$. So $X_{0} / S$ is finite; it follows that $X X / S$ is finite, so $X X=S \cup \bigcup_{\nu=1}^{k}\left(X X \cap c_{i} S\right)$ for some $c_{1}, \ldots, c_{\nu}$. Since $S$ is $\bigwedge$-definable, so is each $c_{i} S$, and we see that the complement of $S$ in $X X$ is also $\Lambda$-definable. When a subset of a definable set and its complement are both $\bigwedge$-definable, they are both definable. Hence $S$ is a definable group. But finitely many cosets of $S$ cover $X$. This contradiction proves the corollary.

Though we stated Proposition 3.12 for finite $X$, it holds with the same proof if the hypothesis $\left|X X^{-1} X\right| \leq k|X|$ is replaced by $\mu\left(\left(X \cup X^{-1}\right)^{3}\right) \leq k \mu(X)$, with $\mu$ an arbitrary right-invariant finitely additive measure on $\widetilde{G}$, or even in the above sense on $\left(X \cup X^{-1}\right)^{3}$.

\section{NeAR-SubGroups AND Lie Groups}

Let $X \subseteq \widetilde{G}$ be a near-subgroup with respect to an $M$-invariant, right-invariant ideal $\mu$, as in the previous section.

Any compact neighborhood $X$ in a Lie group $L$ is (obviously) an approximate subgroup and a near-subgroup with respect to Haar measure. We will show that all 
near-subgroups are related to these classical ones. We will use logical compactness to connect to the locally compact world, and then the Gleason-Yamabe structure theory for locally compact groups in order to find Lie groups.

4.1. Some preliminaries. We will require the following statement: every locally compact group $G$ has an open subgroup $G_{1}$ which is isomorphic to a projective limit of Lie groups. (Gleason defines a topological group $G$ to be a generalized Lie group if for every neighborhood $U$ of the identity there is an open subgroup $H$ of $G$ and a compact normal subgroup $C$ of $H$ such that $C \subseteq U$ and $H / C$ is a Lie group ([15], Definition 4.1). According to [55], Theorem $5^{\prime}$, every locally compact group is a generalized Lie group. By [15, Lemma 4.5, if $G$ is a generalized Lie group with connected component $G^{0}$ of the identity, and $G / G^{0}$ is compact, then $G$ is a projective limit of Lie groups. Now $G / G^{0}$ is totally disconnected. So there exists an open subgroup $G_{1}$ of $G$ containing $G^{0}$, such that $G_{1} / G^{0}$ is compact. Hence $G_{1}$ is an open subgroup of $G$, and a projective limit of Lie groups.)

We will also use the fact that in a connected Lie group $G$, for any chain $C_{1} \subset$ $C_{2} \subset \cdots$ of compact normal subgroups, $\operatorname{cl}\left(\bigcup_{n} C_{n}\right)$ is also a compact normal subgroup. Indeed the dimension of the Lie algebras of the $C_{n}$ must stabilze, so they are locally equal, and hence the connected components $C_{n}^{0}$ stabilize. Factoring out the compact normal subgroup $\bigcup_{n} C_{n}^{0}$, we may assume the $C_{n}$ are discrete, i.e. finite. Since $G$ is connected, the $C_{n}$ are contained in the center $Z$. The connected component $Z^{0}$ of $Z$ has universal covering group $\mathbb{R}^{n}$, so $Z^{0} \cong \mathbb{R}^{k} \oplus(\mathbb{R} / \mathbb{Z})^{l}$. The discrete group $Z / Z^{0}$ is a homomorphic image of the fundamental group of $G / Z$, hence is finitely generated. It has a finite torsion part $A / Z^{0}$; since $Z^{0}$ is divisible, $A$ can be written as a direct sum $A_{0} \oplus Z^{0}$. It is clear that the torsion points of $Z$, and hence all the $C_{n}$, are contained in the compact central subgroup $A_{0} \oplus(\mathbb{R} / \mathbb{Z})^{l}$.

In particular any closed subgroup contains a unique maximal compact normal subgroup of $G$.

Further down (Lemma 6.6), we will also need to know that a compact Lie group has no infinite descending sequences of closed subgroups; this follows easily along the same lines.

The results of this section will also be valid for local groups, using the following local version of Gleason-Yamabe due to Goldbring: for a compact local group $G$ there exist a continuous map $h: D \rightarrow L$ into a Lie group $L$, whose domain $D=D^{-1}$ is a smaller compact neighborhood of 1 in $G$, and whose image $h D$ is a compact neighborhood of 1 in $L$, such that $x y$ is defined for any $x, y \in D$, and we have: $x y \in D$ iff $h(x) h(y) \in h D$, in which case $h(x y)=h(x) h(y)$. [16] has generalized the "no-small-subgroups" theory to the local group setting; to apply it one needs to know that some neighborhood of $1 \in G$ contains a compact normal subgroup, such that the quotient has no small subgroups. This is Lemma 9.3 of [16].

Recall that we call two subsets $X, X^{\prime}$ of a group commensurable if each one is contained in finitely many right translates of the other. If $H, H^{\prime}$ are subgroups, and $H$ is contained in finitely many cosets of $H^{\prime}$, then it is contained in the same number of cosets of $H \cap H^{\prime}$, so $\left[H: H \cap H^{\prime}\right]<\infty$; thus for groups this coincides with the usual notion.

Theorem 4.2. Let $X$ be a near-subgroup of $G$, generating a group $\widetilde{G}$. Then there exists a $\bigvee$-definable subgroup $\breve{G}$ contained in $\widetilde{G}$, an $\bigwedge$-definable subgroup $K \subseteq \breve{G}$, a connected, finite-dimensional Lie group $L$, with no nontrivial normal compact 
subgroups, and a homomorphism $h: \breve{G} \rightarrow L$ with kernel $K$ and dense image, with the following property:

If $F \subseteq F^{\prime} \subseteq L$ with $F$ compact and $F^{\prime}$ open, then there exists a definable $D$ with $h^{-1}(F) \subset D \subset h^{-1}\left(F^{\prime}\right)$. Any such $D$ is commensurable to $X^{-1} X$.

$\breve{G}$ and $K$ are defined without parameters. The Lie group $L$ is uniquely determined.

Let us bring out some facts implicit in the statement of the theorem (and also visible directly in the proof).

Remark 4.3. - If $\left(G^{\prime}, X^{\prime}\right)$ is a countably saturated elementary extension of $(G, X)$, then $h$ extends to $h^{\prime}: \breve{G}^{\prime} \rightarrow L$, and $h^{\prime}$ is surjective.

- The Lie group $L$ is determined up to isomorphism by $(\widetilde{G}, \cdot, X)$, where $\widetilde{G}$ is the subgroup of $G$ generated by $X$, in fact by the theory of $(\widetilde{G}, \cdot, X)$, with $\widetilde{G}$ viewed as many-sorted. We call it the associated Lie group.

- Since any compact subset of a Lie group is a countable intersection of open sets, it follows that if $W \subseteq L$ is compact, then $h^{-1}(W)$ is $\bigwedge$-definable.

- Similarly, if $W \subseteq L$ is open, then $h^{-1}(W)$ is $\bigvee$-definable.

- If $W \subseteq L$ is a neighborhood of 1 , then $h^{-1}(W)$ contains a definable set of the form $U^{-1} U$, with $U$ a definable subset of $(G, X)$ contained in $\breve{G}$ and commensurable to $X^{-1} X$.

- Any definable set containing $K$ contains some $h^{-1}(W)$, with $W$ a neighborhood of 1 in $L$.

- If $L$ is trivial, taking $F=F^{\prime}=L$ in the statement of the theorem we see that $\breve{G}$ is a definable group, commensurable to $X^{-1} X$.

- We have $K \subseteq\left(X X^{-1}\right)^{m}$ for some $m$. Theorem 3.5 provides an $\bigwedge$-definable stabilizer contained in $\left(X^{-1} X\right)^{2}$, but converting it to a 0 -definable one involves some (finite) enlargement.

We first show that the main statement of Theorem 4.2 holds after saturation and base change.

Lemma 4.4. Let $X$ be a near-subgroup of $G$, generating a group $\widetilde{G}$. Assume the structure $(G, X, \ldots)$ is countably saturated. Then over parameters there exists a $\bigvee$ definable subgroup $\breve{G}$ contained in $\widetilde{G}$, an $\bigwedge$-definable subgroup $K \subseteq \breve{G}$, a connected, finite-dimensional Lie group $L$ and a homomorphism $h: \breve{G} \rightarrow L$ with kernel $K$ and dense image, with the following property:

If $F \subseteq F^{\prime} \subseteq L$ with $F$ compact and $F^{\prime}$ open, then there exists a definable $D$ with $h^{-1}(F) \subset D \subset h^{-1}\left(F^{\prime}\right)$. Any such $D$ is commensurable to $X^{-1} X$.

Proof. Let $\widetilde{G}$ be the subgroup of $G$ generated by $X$; let $S_{0}=X X^{-1}$. Theorem 3.5 (via Corollary [3.6) provides definable subsets $S_{n} \subseteq\left(X X^{-1}\right)^{2}$ of $\widetilde{G}$ such that $S=$ $\bigcap_{n \in \mathbb{N}} S_{n}$ is a normal subgroup of $\widetilde{G}$ of bounded index; we may take $S_{n+1}=S_{n+1}^{-1}$ and $S_{n+1} S_{n+1} \subseteq S_{n}$. Define $S_{n}$ for negative $n$ too by $S_{n}=S_{n+1} S_{n+1}$. So $S_{0}$ is 0-definable, and $\bigcup S_{n}=\widetilde{G}$.

We define a topology on $\widetilde{G} / S$ using the quotient map $h: \widetilde{G} \rightarrow \widetilde{G} / S$ by:

(*) $W \subset \widetilde{G} / S$ is closed iff $h^{-1}(W) \cap S_{n}$ is $\bigwedge$-definable for each $n$.

See [25], Section 7 for a more detailed description. Let $L_{0}=\widetilde{G} / S$. This is easily seen to be a locally compact topological group. Compactness is an immediate consequence of saturation and logical compactness: an intersection of a small number 
of $\bigwedge$-definable subsets can never be empty, unless a finite subintersection is empty. Continuity of the group operations follows from the definability of the group structure on $G$. The images of the sets $S_{n}$ form a neighborhood basis for the identity of $L_{0}$ as noted below, so that $L_{0}$ is Hausdorff.

Let $\pi_{S}: \widetilde{G} \rightarrow \widetilde{G} / S$ be the projection. Note that $\pi_{S}^{-1} \pi_{S}\left(S_{n}\right)=\bigcap_{m} S_{n} S_{m} \subseteq$ $S_{n} S_{n}$. In particular $\pi_{S}^{-1} \pi_{S}\left(S_{n}\right)$ is contained in a definable subset of $\widetilde{G}$. In fact for any definable set $D \subseteq S_{n}, \pi_{S}^{-1} \pi_{S}(D)=S D$ is an $\bigwedge$-definable subset of $S_{n-1}$. More generally for any locally definabl $\oint_{1}^{1}$ subset $D$ of $\widetilde{G}, \pi_{S}(D)$ is closed. Indeed $\pi_{S}^{-1} \pi_{S}(D) \cap S_{n}=\pi_{S}^{-1}\left(\pi_{S}\left(D \cap S_{n} S_{n}\right)\right)$.

In particular, the image of $\widetilde{G} \backslash S_{n} S_{n}$ in $\widetilde{G} / S$ is closed, and disjoint from $\pi_{S}\left(S_{n}\right)$ since $\pi_{S}\left(S_{n} S_{n}\right) \cup \pi_{S}\left(\widetilde{G} \backslash S_{n} S_{n}\right)=\widetilde{G} / S, \pi_{S}\left(S_{n}\right)$ lies in the interior of $\pi_{S}\left(S_{n} S_{n}\right)$. In particular, each $\pi_{S}\left(S_{n} S_{n}\right)$ is a neighborhood of 1 , as is theorefore $\pi_{S}\left(S_{n+1}\right)$.

By Yamabe, $L_{0}$ has an open subgroup $\breve{G} / S$, isomorphic to a projective limit of Lie groups. $\breve{G} / S$ is also closed, so both $\breve{G} \cap D$ and $D \backslash \breve{G}$ are $\bigwedge$-definable, for any definable $D$ contained in $\widetilde{G}$. Thus $\breve{G}$ is locally definable in $\widetilde{G}$; i.e., it has a definable intersection with any definable subset of $\widetilde{G}$.

The topology of a projective limit $\lim L_{i}$ is generated by pullbacks of open subsets of individual factors $L_{i}$. So there exist a Lie group $L$, a neighborhood $U_{1}$ of the identity in $L$, and a homomorphism $h: \breve{G} / S \rightarrow L$, such that $h^{-1}\left(U_{1}\right) \subseteq \pi_{S}\left(S_{1}\right)$. By shrinking $\breve{G}$ down further to the pullback of the (open) connected component of 1 in $L$, we can take $L$ to be connected. Let $\pi: \breve{G} \rightarrow \breve{G} / S \rightarrow L$ be the composition.

Now $(*)$ holds for $L$ : the morphism from a projective limit to one of the factors is closed; so $Y \subseteq L$ is closed iff $h^{-1}(Y)=\pi^{-1}(Y) / S$ is closed iff $\pi^{-1}(Y)$ meets every definable set in an $\Lambda$-definable set.

We also have:

(**) For any compact neighborhood $U$ of 1 in $L, \pi^{-1}(U)$ is commensurable to $X^{-1} X$.

This is the case because any two compact neighborhoods of 1 in $L$ are commensurable, each one being contained in a union of translates of the other, which can be reduced by compactness to a finite union. This comparability is preserved by $\pi^{-1}$. So it suffices to show that $\pi^{-1}(U)$ contains $X^{-1} X$ for some $U$, and that $\pi^{-1}\left(U^{\prime}\right)$ is contained in finitely many translates of $X^{-1} X$ for some $U^{\prime}$. On the other hand by the Ruzsa argument (above Lemma 3.3), any $S_{n}$ is commensurable to $X^{-1} X$. We saw that $\pi_{S}\left(X^{-1} X\right)$ is compact; hence $\pi\left(X^{-1} X\right)$ is compact, so it is contained in some compact open neighborhood $U$, and thus $X^{-1} X \subseteq \pi^{-1}(U)$. By construction, $\pi^{-1}\left(U_{1}\right)=\pi_{S}^{-1} h^{-1}\left(U_{1}\right) \subseteq S_{1}$, giving the second direction.

If $F$ is a compact subset of $L$ and $F^{\prime}$ is an open subset, with $F \subset F^{\prime}$, then there exists a definable $D$ with $h^{-1}(F) \subset D \subset h^{-1}\left(F^{\prime}\right)$. Indeed $\pi^{-1}(F)$ is an $\bigwedge$-definable set contained in the $\bigvee$-definable set $\pi^{-1}\left(F^{\prime}\right)$, so there exists a definable $D$ with $\pi^{-1}(F) \subseteq D \subseteq \pi^{-1}\left(F^{\prime}\right)$.

We now begin to address the issue of parameters.

Lemma 4.5. With the assumptions and notation of Lemma 4.4, there exists an $\bigwedge$-definable subgroup $S$ of $\widetilde{G}$ without parameters, with $\widetilde{G} / S$ bounded.

${ }^{1}$ See the definition in the first lines of $\S 3$. 
Proof. We may work in a homogeneous elementary extension $\mathbb{U}$ of $(G, X, \cdot)$, so that $\bigwedge$-definable sets are $\Lambda$-definable without parameters as soon as they are Aut(U)invariant.

Let $\alpha$ be the set of pairs $(H, \Gamma)$ such that $\Gamma \leq H \leq \widetilde{G}$, and for some small base $A$, $\Gamma$ is a normal subgroup of $H, \Gamma$ is $A$ - $\bigwedge$-definable, $H$ is a locally definable subgroup of $\widetilde{G}$ over $A$, and $\widetilde{G} / \Gamma$ is bounded. Let $\beta$ be the set of pairs $(H, \Gamma) \in \alpha$ such that if $\left(H^{\prime}, \Gamma^{\prime}\right) \in \alpha$ and $\Gamma \leq \Gamma^{\prime} \leq H^{\prime} \leq H$, then $H=H^{\prime}$ and $\Gamma=\Gamma^{\prime}$. Equivalently, the locally compact group $H / \Gamma$ is connected, with no nontrivial compact normal subgroups. (Hence, by Yamabe, $H / \Gamma$ is a Lie group.)

$\operatorname{For}(H, \Gamma) \in \beta$ it is clear that $H$ determines $\Gamma$, since if $\left(H, \Gamma^{\prime}\right) \in \beta$ then $\Gamma=$ $\Gamma \Gamma^{\prime}=\Gamma^{\prime}$.

Claim $1 . \beta$ is nonempty.

Proof. We saw above that there exists $(H, \Gamma) \in \alpha$ with $H / \Gamma$ a connected Lie group. In the preliminaries to this section we saw that $H / \Gamma$ has a maximal compact normal subgroup; it has the form $H / \Gamma^{\prime}$ with $\Gamma^{\prime} \wedge$-definable. Then $\left(H, \Gamma^{\prime}\right)$ is in $\beta$.

Claim 2. Let $(H, \Gamma),\left(H^{\prime}, \Gamma^{\prime}\right) \in \beta$. Then $\left(H \cap H^{\prime}, \Gamma \cap H^{\prime}\right) \in \beta$.

Proof. Since $H^{\prime}$ is locally definable, while $\Gamma$ is contained in a definable set, it is clear that $H^{\prime} \cap \Gamma$ is $\bigwedge$-definable. Since $\widetilde{G} / \Gamma$ and $\widetilde{G} / H^{\prime}$ are bounded, so is $\widetilde{G} /\left(\Gamma \cap H^{\prime}\right)$. Also $H \cap H^{\prime}$ is locally definable. Thus $\left(H \cap H^{\prime}, \Gamma \cap H^{\prime}\right) \in \alpha$.

Now $\Gamma^{\prime} /\left(\Gamma^{\prime} \cap H\right)$ is bounded (as it embeds into $\left.\widetilde{G} / H\right)$. By Lemma 1.6, $\Gamma^{\prime} \cap H$ has finite index in $\Gamma^{\prime}$.

Similarly, $\Gamma$ is contained in finitely many cosets of $H^{\prime}$, hence of $H^{\prime} \cap H$. So $\Gamma\left(H^{\prime} \cap H\right)$ is a finite union of cosets of $H^{\prime} \cap H$, and hence is a locally definable subgroup of $H$. We saw that for any definable set $D$ containing $\Gamma$, the image of $D^{-1} D$ contains an open neighborhood of the identity. Hence the image of $\Gamma\left(H^{\prime} \cap H\right)$ in $H / \Gamma$ is open.

Now the natural map $\left(H \cap H^{\prime}\right) /\left(\Gamma \cap H^{\prime}\right) \rightarrow H / \Gamma$ is injective. But it has open image and the group $H / \Gamma$ is connected, so the map is surjective. Thus $\left(H \cap H^{\prime}\right) /(\Gamma \cap$ $\left.H^{\prime}\right) \cong H / \Gamma$ and hence has no nontrivial compact normal subgroups.

Similarly $\left(H \cap H^{\prime}, \Gamma^{\prime} \cap H\right) \in \beta$. So $\Gamma^{\prime} \cap H=\Gamma \cap H^{\prime}$ and thus $\Gamma^{\prime} \cap H=\Gamma \cap \Gamma^{\prime}$.

We noted that $\Gamma^{\prime} \cap H$ has finite index in $\Gamma^{\prime}$; moreover since this holds for any pair from $\alpha$, in particular it holds for $\Gamma^{\prime}$ and any Aut(U)-conjugate $\sigma(H)$ of $H$, so $\Gamma^{\prime} \cap \sigma(H)$ has index bounded independently of $\sigma$.

So $\Gamma \cap \sigma\left(\Gamma^{\prime}\right)$ has finite index in $\Gamma^{\prime}$, bounded independently of $\sigma \in \operatorname{Aut}(\mathbb{U})$. By symmetry, $\Gamma, \Gamma^{\prime}$ are commensurable, and all conjugates of $\Gamma$ are uniformly commensurable.

Pick $\Gamma_{1} \in \beta$. By [2], there exists an Aut(U)-invariant group $S_{1}$ commensurable to each conjugate of $\Gamma_{1}$. The proof of 2 shows that $S_{1}$ contains a finite intersection of conjugates of $\Gamma_{1}$ as a subgroup of finite index; so $S_{1}$ is $\Lambda$-definable, and of bounded index in $\widetilde{G}$. Being Aut(U)-invariant, it is $\Lambda$-definable over $\emptyset$. Let $S$ be the intersection of all $\widetilde{G}$-conjugates of $S_{1}$; then $S$ is normal in $\widetilde{G}, \wedge$-definable over $\emptyset$, and of bounded index.

Proof of Theorem 4.2. We may assume that $(G, X)$ is countably saturated, since the statements descend from a saturated extension of $(G, X)$ to $(G, X)$ by restriction, using the same (0-definable) $\breve{G}, h, L$. Let $S$ be the 0 - $\bigwedge$-definable group given 
by Lemma 4.4. Since $\beta \neq \emptyset$ in Lemma 4.4, we know that $\breve{G}, h$ exist over parameters, and it remains only to show that $\breve{G}$ and $\operatorname{ker}(h)$ can be chosen to be $\bigvee$-definable and $\bigwedge$-definable (respectively) without parameters.

We begin with $\breve{G}$. We may replace $\breve{G}$ by the pre-image of any open subgroup of $\breve{G} / S$ (the "connected-by-compact" condition will remain valid). Let $G_{c}$ be the group generated by $\breve{G} \cap S_{1}$. Note that $G_{c}$ is locally definable, and is generated by $Z_{c}=G_{c} \cap S_{1}$; so each of $G_{c}, Z_{c}$ can be used to define the other. Now $Z_{c}$ is a definable set, with parameter $c$ say. Let $Q$ be the set of realizations of $t p(c)$. If $c^{\prime} \in Q$, then $Z\left(c^{\prime}\right)$ generates a group $G_{c^{\prime}}$, and $G_{c^{\prime}} \cap S_{1}=Z\left(c^{\prime}\right)$. Thus for $c^{\prime \prime}, c^{\prime} \in Q$, $G_{c^{\prime \prime}}=G_{c^{\prime}}$ iff $Z_{c^{\prime \prime}}=Z_{c^{\prime}}$; this is a definable equivalence relation.

We have $h: \breve{G} \rightarrow L$. Note that if $C$ is a compact normal subgroup of $L$, the composition of $h$ with the quotient map $L \rightarrow L / C$ has the same properties as $h: \breve{G} \rightarrow L$. Replacing $L$ by $L / C$ for a maximal compact normal subgroup $C$ of $L$, we may assume that $L$ has no compact normal subgroups.

Let $K$ be the kernel of $h$. Then $K$ is $\operatorname{Aut}(\mathbb{U})$-invariant. For if $K^{\prime}$ is an $\operatorname{Aut}(\mathbb{U})$ conjugate of $K$, then $K, K^{\prime}$ are $\bigwedge$-definable normal subgroups of $\breve{G} . K^{\prime} K / K$ is a compact normal subgroup of $\breve{G} / K$, hence it is trivial, and similarly $K^{\prime} K / K^{\prime}$ is trivial, so $K=K^{\prime}$. Thus $K$ is $\bigwedge$-definable without parameters.

It remains to prove the uniqueness of $L$. Let us compare $L$ to the locally compact group $\widetilde{H}:=\widetilde{G} / S$, where $S=\widetilde{G}_{\emptyset}^{00}$ is the smallest 0 - $\bigwedge$-definable subgroup of $\widetilde{G}$ of bounded index. Let $H$ be the image of $\breve{G}$ in $\widetilde{H}$. Then $H$ is an open subgroup of $\widetilde{H}$, so the connected component of the identity $\widetilde{H}^{0}$ is contained in $H$, and equals $H^{0}$. Let $C$ be the image of $K$ in $\widetilde{H}$. So $C$ is a normal subgroup of $H$. Since $H / C$ is connected, we have that $H /\left(C \widetilde{H}^{0}\right)$ is both connected and totally disconnected. (Unlike the situation in the category of topological spaces, in the category of topological groups the image of a totally disconnected group is still totally disconnected. Indeed it has a pro-finite open subgroup, and this remains the case for a quotient group.) So $C H^{0}=H$. Both $C$ and $H^{0}$ are normal in $H$, so letting $C_{0}=C \cap H^{0}$ we have $H / C_{0} \cong C / C_{0} \times H^{0} / C_{0}$. Thus the action of $C$ by conjugation on $H^{0}$ is trivial modulo $C_{0}$. Now $C$ is a maximal normal compact subgroup of $H ; C_{0}$ is a compact normal subgroup of $H_{0}$, maximal with respect to being normalized by $C$ too, but we have just shown that this last condition is trivial, so $C_{0}$ is a maximal compact normal subgroup of $H_{0}$. We have $L=H / C \cong H^{0} / C_{0}$ canonically. Now it is clear that $C_{0}$ is the unique maximal normal compact subgroup of $H^{0}$. (If $C_{1}$ were another, $C_{0} C_{1}$ would be still bigger.) This proves the uniqueness of $L$.

If we expand $(\widetilde{G}, X, \cdot, \ldots)$ to a structure $\underline{\widetilde{G}}=\left(\widetilde{G}, X, \cdot, \ldots, R_{\text {new }}, \ldots\right)$ with more definable sets, the smallest 0 - $\bigwedge$-definable subgroup of bounded index may become smaller: $\widetilde{G}_{0}^{00} \subset \widetilde{G}_{0}^{00}$. Thus $H=\widetilde{G} / S, H^{0}, C_{0}$ will change with the added structure. Nevertheless the isomorphism proved in the last paragraph of the proof remains valid; hence the associated Lie group $L=H^{0} / C_{0}$ does not change if the structure is enriched.

Definition 4.6. Let $\widetilde{G}$ be a $\bigvee$-definable group, $X$ a definable near-subgroup of $\widetilde{G}$, generating $\widetilde{G}$. Let $M=(\widetilde{G}, X, \cdot, \ldots)$, where $\ldots$ indicates possible additional structure.

- $L C(M)=\widetilde{G} / S$, where $S$ is the smallest $\bigwedge$-definable subgroup of $\widetilde{G}$, without parameters, of bounded index. 
- $L(X)$ is the Lie group associated to $X$; so $L(X)=L C(M)^{0} / C_{0}$, with $C_{0}$ a maximal normal compact subgroup of $L C(M)^{0}$. Let $\widehat{L}(X)=L C(M) / C_{0}$; then $L(X)=\widehat{L}(X)^{0}$, the connected component.

- $l(X)=\operatorname{dim} L(X)$.

We refer to $l(X)$ as the Lie rank of $X$, or of $\widetilde{G}$.

Example 4.7. If a near-subgroup $X$ has $l(X)=0$, then there exists a definable group $S$ with $X, S$ commensurable. Indeed in this case kernel $S$ of the homomorphism $\breve{G} \rightarrow L$ is equal to $\breve{G}$; but $S$ is $\bigwedge$-definable and $\breve{G}$ is $\bigvee$-definable, so they are definable.

Lemma 4.8. In the situation of Theorem 4.2, assume that the S1-ideal arises from an invariant, translation invariant measure $\mu$. Let $k_{5}=\mu\left(X X^{-1} X X^{-1} X\right) / \mu(X)$. Extend $\mu$ to the $\sigma$-algebra generated by the $\infty$-definable subsets of $\widetilde{G}$, and let $\lambda$ be the pushforward of $\mu$ to $\widehat{L}=\widehat{L}(X)$, i.e. $\lambda(U)=\mu\left(\pi^{-1}(U)\right) \in \mathbb{R}_{\infty}$, where $\pi$ is the quotient map. Then $\lambda$ is a Haar measure on $\widehat{L}$. We have $\lambda\left((\pi X)(\pi X)^{-1}(\pi X)\right) \leq$ $k_{5} \lambda(\pi X)$. Moreover, there exists a compact subset $W$ of $L=L(X)$ with $\lambda(W)>0$ and $\lambda\left(W W^{-1} W\right) \leq k_{5} \lambda(W)$. We can take $1 \in W$.

Proof. It is clear that $\lambda$ is a nonzero, translation invariant measure, hence a Haar measure. We have $X X^{-1} X \subseteq \pi^{-1}\left((\pi X)(\pi X)^{-1}(\pi X)\right) \subseteq\left(X X^{-1} X X^{-1} X\right)$, since $\pi^{-1}(1) \subseteq X^{-1} X$. This implies the first inequality, by definition of the pushforward measure. Moving to $L$, recall that we have $h: \breve{G} \rightarrow L$ with kernel $K$ (Theorem 4.2), with $\breve{G}$ a $\bigvee$-definable subgroup of $\widetilde{G}$, and $\widetilde{G} / \breve{G}$ bounded. In particular $X / \breve{G}$ is bounded, so $X$ intersects finitely many cosets of $\breve{G}$; say $X=\bigcup_{i=1}^{r} X_{i}$, with $X_{i} \subseteq$ $c_{i} \breve{G}$, and $c_{i}$ lying in distinct cosets of $\breve{G}$. Let $k_{3}=\mu\left(X X^{-1} X\right) / \mu(X)$. Then, noting that $X_{i} X_{i}^{-1} X_{i} \subseteq c_{i} \breve{G}$, and the $c_{i} \breve{G}$ are disjoint, we have:

$$
\sum_{i} \mu\left(X_{i} X_{i}^{-1} X_{i}\right) \leq \mu\left(X X^{-1} X\right) \leq k_{3} \mu(X)=\sum_{i} k_{3} \mu\left(X_{i}\right) .
$$

The sum being extended over all $i \leq r$ such that $\mu\left(X_{i}\right)>0$, it follows that for at least one $i$ with $\mu\left(X_{i}\right)>0$, we have $\mu\left(X_{i} X_{i}^{-1} X_{i}\right) \leq k_{3} \mu\left(X_{i}\right)$. Similarly, for at least one $i$ with $\mu\left(X_{i}\right)>0$, we have $\mu\left(X_{i} X_{i}^{-1} X_{i} X_{i}^{-1} X_{i}\right) \leq k_{5} \mu\left(X_{i}\right)$. Let $Y=c_{i}^{-1} X_{i}$. Then $h(Y)$ is a compact subset of $L ; \lambda(h(Y))=\mu\left(h^{-1} h(Y)\right) \geq \mu(Y)>0$ and $\lambda\left(Y Y^{-1} Y\right) \leq k_{5} \lambda(Y)$ by the same argument as for $\widehat{L}$ above. By translating $W$, we can arrange $1 \in W$.

Can Lemma 4.8 be used to bound $l(X)=\operatorname{dim}(L)$ in terms of doubling constants of $X$ ? When $G$ is nilpotent, we have: $l(X) \leq \log _{2}\left(k_{5}\right)$. This follows from Lemma 4.8 and Lemma 4.9, due (with a different proof) to Tsachik Gelander; thanks for allowing me to include it here. Use $1 \in W$ to obtain $W W \subseteq W W^{-1} W$ in order to apply the lemma.

Lemma 4.9 (Gelander). Let $X$ be a compact subset of $\mathbb{R}^{d}$, or more generally of a connected, simply connected Lie group, and let $\lambda$ be Haar measure. Then $\lambda(X X) \geq 2^{d} \lambda(X)$.

Proof. In fact we have $\lambda(s(X)) \geq 2^{d} \lambda(X)$, where $s(x)=x^{2}$. The ambient group $H$ is isomorphic to a subgroup of the strict upper triangular matrices over $\mathbb{R}$, of some dimension; the map $s$ is hence injective. Moreover $H$ is diffeomorphic to $\mathbb{R}^{d}$, and 
the differential $d s$ of $s$ at any point is a linear transformation of the form $2+M$, with $M$ nilpotent. It follows that the Jacobian determinant has value $2^{d}$, so by the change of variable formula for integration, the diffeomorphism $s$ expands volume by exactly $2^{d}$.

Remark 4.10. (Compare [50, Lemma 7.7 and Theorem 7.12.)

Let $\Gamma$ be a $\Lambda$ - definable subgroup of bounded index in the $\bigvee$-definable group $\widetilde{G}$. Let $\widetilde{N}$ be a locally definable normal subgroup of $\widetilde{G}$, and let $\pi: \widetilde{G} \rightarrow \widetilde{G} / \widetilde{N}$ be the quotient map. The main case is that $\widetilde{N}$ is the intersection with $\widetilde{G}$ of a definable normal subgroup $N$ of $G$.

(0) The image $\boldsymbol{\Gamma}$ of $\Gamma$ has bounded index in the image $\mathbf{G}$ of $\widetilde{G}$ modulo $\widetilde{N}$, and also $\Gamma \cap \widetilde{N}$ has bounded index in $\widetilde{N}$. Conversely in this situation the boundedness of $\widetilde{G} / \Gamma$ follows from that of $\mathbf{G} / \boldsymbol{\Gamma}$ and of $\Gamma \cap \widetilde{N}$ in $\widetilde{N}$.

(1) View $\widetilde{G} / \Gamma, \mathbf{G} / \boldsymbol{\Gamma}$ and $\widetilde{N} /(\Gamma \cap \widetilde{N})$ as locally compact groups. Then $\widetilde{N} /(\Gamma \cap \widetilde{N})$ with the logic topology is homeomorphic to the image of $\widetilde{N}$ in $\widetilde{G} / \Gamma$, with the subspace topology. Indeed the natural map $\widetilde{N} /(\Gamma \cap \widetilde{N}) \rightarrow \widetilde{G} / \Gamma$ is a continuous injective homomorphism. To see that it is also a closed map, since $\widetilde{G} / \Gamma$ is covered by the interiors of sets of the form $\pi(D)$, with $D$ definable, we may restrict attention to the inverse image of such a set. But then we are looking at an injective continuous map between compact Hausdorff spaces, hence an isomorphism.

Similarly, $\mathbf{G} / \boldsymbol{\Gamma} \cong(\widetilde{G} / \Gamma) /(\widetilde{N} /(\Gamma \cap \widetilde{N}))$ as topological groups.

(2) $\Gamma$ is definable iff the topology on $\widetilde{G} / \Gamma$ is discrete. This makes it plain that $\Gamma$ is definable iff $\pi(\Gamma)$ and $\Gamma \cap \widetilde{N}$ are.

(3) If $\widetilde{G} / \Gamma$ is a Lie group, then so are $\widetilde{N} /(\Gamma \cap \widetilde{N})$ and $\mathbf{G} / \boldsymbol{\Gamma}$, and we have an exact sequence

$$
1 \rightarrow \tilde{N} /(\Gamma \cap \tilde{N}) \rightarrow \widetilde{G} / \Gamma \rightarrow \mathbf{G} / \mathbf{\Gamma} \rightarrow 1 .
$$

This in turn induces an exact sequence of homomorphisms among the Lie algebras. It follows that $\operatorname{dim}(G / \Gamma)=\operatorname{dim}(\mathbf{G} / \boldsymbol{\Gamma})+\operatorname{dim}(\widetilde{N} /(\Gamma \cap \widetilde{N}))$.

(4) From (3) it follows that

$$
l(\widetilde{G}) \geq l(\widetilde{G} / \widetilde{N})+l(\widetilde{N}) .
$$

Indeed we may move from $\widetilde{G}$ to $\mathbf{G}$, changing none of the three numbers. Then we may enlarge $\Gamma$ so that $\mathbf{G} / \Gamma$ has no nontrivial normal compact subgroups. By (3) we obtain in this situation: $l(\widetilde{G})=\operatorname{dim}(\mathbf{G} / \boldsymbol{\Gamma})+\operatorname{dim}(\widetilde{N} /(\Gamma \cap \tilde{N}))$. Now $\widetilde{N} /(\Gamma \cap \widetilde{N})$ may have nontrivial compact subgroups, but we have at all events $l(\widetilde{N}) \leq \operatorname{dim}(\widetilde{N} /(\Gamma \cap$ $\widetilde{N}))$ (the inequality may be strict). Similarly $l(\widetilde{G} / \widetilde{N}) \leq \operatorname{dim}(\widetilde{G} / \widetilde{N})$, and (4) follows.

See $\S 7$ for an inductive use of this invariant, similar to Gromov's use of the growth rate in the case of his polynomial growth assumption.

Remark 4.11. The canonicity of $L$ in Theorem 4.2 is achieved at a price. We noted already that it requires moving from $\left(X^{-1} X\right)^{2}$ to $\left(X^{-1} X\right)^{m}$, where $m$ is difficult to control. In addition, factoring out the maximal compact normal subgroup can lead to substantial loss of information.

In some cases there will exist a largest $\bigwedge$-definable normal subgroup $\Delta$ of $\widetilde{G}$ with $\Delta \subseteq X^{-1} X$. By Yamabe, $\widetilde{G} / \Delta$ is a Lie group $\widetilde{L}$. In this case $\widetilde{L}$ too is an invariant of $(\widetilde{G}, X)$ and is superior in both respects. When it exists, we may call $\widetilde{L}$ 
the directly associated Lie group. More generally we may need to look at a number of $\widetilde{G} / \Delta$, differing by compact isogenies.

For example, let $\alpha>10$ be an irrational real number, and let

$$
X[n]=X[n, \alpha]=\{[m \alpha]: m \in \mathbb{Z},-n \leq m \leq n\},
$$

where $[m \alpha]$ is the integer part of $m \alpha . X[n]$ is symmetric, and satisfies $|X[n] X[n]| /$ $|X[n]| \leq 4$. Let $\left(G, X, n^{*}\right)$ be a nonprincipal ultraproduct of $(\mathbb{Z}, X[n], n)$. Then the directly associated Lie group is the product of the circle $\mathbb{R} / \alpha \mathbb{Z}$ with $\mathbb{R}$. The map $\widetilde{G} \rightarrow \mathbb{R}$ takes $x$ to the standard part of $x / n^{*}$. The map $\widetilde{G} \rightarrow \mathbb{R} / \alpha \mathbb{Z}$ takes $x$ to the standard part of the image of $x$ in the nonstandard circle $\mathbb{R}^{*} / \alpha$. The image of $X$ in the cylinder $\mathbb{R} \times \mathbb{R} / \alpha \mathbb{Z}$ is the image of the square $[-\alpha, \alpha] \times[-1,0]$. The image of the element $[\alpha]$ is $(0, m+\alpha \mathbb{R})$ for some nonzero integer $m$; it follows that $(0) \times \mathbb{R} / \alpha \mathbb{Z}$ is contained in the image of $\widetilde{G}$, so that $\widetilde{G} \rightarrow \mathbb{R} \times \mathbb{R} / \alpha \mathbb{Z}$ is surjective. The doubling of this square within the cylinder is similar to the doubling of the $X[n]$ within $\mathbb{Z}$. By contrast the associated Lie group without compact subgroups is $\mathbb{R}$, which does not account for the doubling of $X$ or $X X$ very well, and only begins to work around the $[\alpha]$ th set power of $X$.

It is also interesting to note here that if one takes $X[n]^{\prime}=\left\{\left[m \alpha_{n}\right]: m \in \mathbb{Z},-n \leq\right.$ $m \leq n\}$, where $\alpha_{n}$ approaches $\infty$, the associated Lie group will be $\mathbb{R}^{2}$; this limit is natural for the directly associated Lie group but not for the reduced one.

For the record we state a version of Theorem 4.2 waiving canonicity but gaining more control of the location of the kernel.

Lemma 4.12. Let $X$ generate a $\bigvee$-definable group $\widetilde{G}$, and assume an ideal on $\widetilde{G}$ exists satisfying the assumption of Lemma 2.17. Then there exists a $\bigvee$-definable subgroup $\breve{G}$ contained in the group generated by $X$, a $\wedge$-definable subgroup $K \subseteq \breve{G}$, a connected, finite-dimensional Lie group $L$ and a homomorphism $h: \breve{G} \rightarrow L$ with kernel $K \subseteq\left(X^{-1} X\right)^{2}$ and dense image, such that:

If $F \subseteq F^{\prime} \subseteq L$ with $F$ compact and $F^{\prime}$ open, then there exists a definable $D$ with $h^{-1}(F) \subset D \subset h^{-1}\left(F^{\prime}\right)$. Any such $D$ is commensurable to $X^{-1} X$.

$\breve{G}, K$ may be defined with parameters in any given model.

Proof. By Lemma 2.17 and Theorem 3.5, one obtains an $\bigwedge$-definable stabilizer $S$ defined over a given model, and with $S \subseteq\left(X^{-1} X\right)^{2}$. It follows that the image $U$ of $\left(X^{-1} X\right)^{2}$ in $\widetilde{G} / S$ contains the identity in its interior. We follow the proof of Theorem 4.2, taking care to factor out only by a compact subgroup contained in the given neighborhood $U$.

In the local group setting, the conclusion reads: there exists a homomorphism $h: W \rightarrow L$ of local groups, $W$ a subset of $X$ commensurable to $X^{-1} X$, such that $\mathbf{X}=h(X)$ is a compact neighborhood of $1 \in L$; and if $F \subseteq F^{\prime} \subseteq \mathbf{X}$ with $F$ compact and $F^{\prime}$ open, then there exists a definable $D$ with $h^{-1}(F) \subset D \subset h^{-1}\left(F^{\prime}\right)$. Any such $D$ is again commensurable to $X^{-1} X$. I have not checked the question of parameters for local groups.

Corollary 4.13. Let $X$ be a near-subgroup of a group $G_{0}$, generating $\widetilde{G}$. Then there exist 0-definable subsets $X_{1}, X_{2}, \ldots$ of $\widetilde{G}$, commensurable to $X^{-1} X$, and $c \in \mathbb{N}$, with:

(1) $1 \in X_{n}=X_{n}^{-1}$.

(2) $X_{n+1} X_{n+1} \subseteq X_{n}$. 
(3) $X_{n}$ is contained in $\leq c$ translates of $X_{n+1}$.

(4) $a X_{n+1} a^{-1} \subseteq X_{n}$ for $a \in X_{1}$.

(5) $\left[X_{n}, X_{m}\right]=\left\{x y x^{-1} y^{-1}: x \in X_{n}, y \in X_{m}\right\} \subseteq X_{k}$ whenever $k<n+m$. In particular each $X_{n}$ is closed under the commutator bracket.

(6) $X_{n+1}=\left\{x \in X_{1}: x^{4} \in X_{n}\right\}$.

(7) Let $x, y \in X_{m}, m \geq 2$ and suppose $x^{2}=y^{2}$. Then $x y^{-1} \in \bigcap_{n} X_{n}$.

Proof. We may assume $\left(G_{0}, X\right)$ is $\aleph_{0}$-saturated. Let $h, L$ be as in Theorem 4.2 We first show that $L$ has a system $U_{n}$ of compact neighborhoods of the identity with properties (1)-(3).

Let $\mathfrak{L}$ be the Lie algebra of $L, \exp : \mathfrak{L} \rightarrow L$ the exponential map, and fix a Euclidean inner product on $\mathfrak{L}$. Let $V$ be a simply connected open neighborhood of $0 \in \mathfrak{L}$ such that $\exp$ is a diffeomorphism $V \rightarrow \exp (V)=U$, and such that the image of $\left(X^{-1} X\right)^{2}$ in $L$ contains $U$ in its interior. Let $V_{n}$ be the ball of radius $r_{0} 2^{-n}$ around 0 . Here $r_{0}>0$ is chosen small enough so that $V_{0}$ is contained in $V$; some further constraints on $r_{0}$ will be specified later. Viewing $\mathfrak{L}$ as the tangent space at 1 of $L$, fix on $L$ the unique left-invariant Riemannian metric extending the given inner product at 1 . Let $U_{n}=\exp \left(V_{n}\right)$. Note that $U_{n}$ is the set of points of $U$ at Riemannian distance $\leq r_{0} 2^{-n}$ from the identity element (cf. e.g. [36], Prop. 6.10). It follows that (1)-(2) hold: $1 \in U_{n}=U_{n}^{-1}$ and $U_{n+1} U_{n+1} \subset U_{n}$.

Fix an invariant volume form $\omega$ on $L$. We claim that for some constant $c^{\prime}>0$, we have $\operatorname{vol}\left(U_{n+1}\right) \geq c^{\prime} \operatorname{vol}\left(U_{n}\right)$ for large enough $n$. We have $\operatorname{vol}\left(U_{n}\right)=\int_{V_{n}} \exp ^{*} \omega$ where $\exp ^{*} \omega$ is the pullback. Now $V_{n}$ has volume proportional to $2^{-n d}$, with respect to the standard Euclidean volume form $\omega_{1}$. We have $\exp ^{*} \omega=f \omega_{1}$ for some nonvanishing smooth function $f$, which we can take to be positive. On $V$ we have $\left(c^{\prime \prime}\right)^{-1} \leq f \leq c^{\prime \prime}$ for some $c^{\prime \prime}>0$, so $\operatorname{vol}\left(U_{n}\right) \leq c^{\prime \prime} \operatorname{vol}\left(V_{n}\right) \leq 2^{d} c^{\prime \prime} \operatorname{vol}\left(V_{n+1}\right) \leq$ $2^{d}\left(c^{\prime \prime}\right)^{2} \operatorname{vol}\left(U_{n+1}\right)$.

Now $U_{n-1}$ contains at most $\operatorname{vol}\left(U_{n-1}\right) / \operatorname{vol}\left(U_{n+2}\right)$ disjoint $U_{n}$ - translates of $U_{n+2}$; hence $U_{n}$ is contained in that many translates of $U_{n+2}^{-1} U_{n+2} \subseteq U_{n+1}$. This gives the analogue of (3).

To obtain (4), we may begin with $r_{1}$ small enough so that for $x \in U_{1}, 1-a d_{x}$ has operator norm $<1 / 2$. Then $a d_{x}\left(V_{n+1}\right) \subseteq V_{n}$, so $x^{-1} U_{n+1} x \subseteq U_{n}$.

(5) Let $c(x, y)=\log (\exp (x) \exp (y) \exp (-x) \exp (-y))$. We have to show that $c\left(V_{n}, V_{m}\right) \subseteq V_{k}$ when $k \leq N, k<n+m$. Now if $|u|<2^{-n}$ and $|v|<2^{-m}$, then $|c(u, v)-[u, v]| O\left(2^{-m-n-\min (m, n)}\right)$, where $[u, v]$ is the Lie algebra bracket. This can be seen by looking at the power series expansion of $c$; it begins with $[u, v]$, followed by higher-order terms. So the statement holds for large enough $m, n$; by renormalizing (replacing $V_{n}$ by $V_{n+k}$ ) we obtain the result.

Finally note that $U_{n+1}=\left\{u \in U_{1}: u^{2} \in U_{n}\right\}$ since for $u=\exp (v)$ we have $u^{2}=\exp (2 v)$ and $u \in U_{n+1}$ iff $v \in V_{n+1}$ iff $2 v \in V_{n}$ iff $u^{2} \in U_{n}$.

Since $h^{-1}\left(U_{2}\right)$ is an $\bigwedge$-definable set contained in the definable set $h^{-1}\left(U_{1}\right)$, there exists a definable set $Y_{1}$ with $h^{-1}\left(U_{2}\right) \subseteq Y_{1} \subseteq h^{-1}\left(U_{1}\right)$. Define $Y_{n}$ inductively by $Y_{n+1}=\left\{y \in Y_{1}: y^{2} \in Y_{n}\right\}$. It follows that $h^{-1}\left(U_{n+1}\right) \subseteq Y_{n} \subseteq h^{-1}\left(U_{n}\right)$. (If $h(x) \in U_{n+1}$, then $h\left(x^{2}\right) \in U_{n}$. By induction $x^{2} \in Y_{n-1} ;$ so $x \in Y_{n}$. If $x \in Y_{n}$, then $x^{2} \in Y_{n-1} \subseteq h^{-1} U_{n-1}$, so $h(x)^{2} \in U_{n-1}$ and $h(x) \in U_{n}$.) Clearly $Y_{n}=Y_{n}^{-1}$. It follows from the intertwining of the $Y_{n}$ in the $h^{-1} U_{n}$ that $Y_{n+2} Y_{n+2} \subseteq Y_{n}$, that $Y_{n}$ is contained in at most $c^{2}$ translates of $Y_{n+1}, a Y_{n+2} a^{-1} \subseteq Y_{n}$, and $\left[Y_{n}, Y_{m}\right] \subseteq Y_{k}$ whenever $k+1<n+m$. 
Let $X_{n}=Y_{2 n}$. Then it is clear that (1)-(6) hold. (7) follows from the fact that squaring is injective on $U_{1}$ (if one chooses $U_{1}$ small enough).

Remark 4.14. (1) Let $\left(X_{n}\right)$ be as in Lemma 4.13. Let $(G, X)$ be a nonprincipal ultraproduct of $\left(G_{0}, X_{n}\right)$, and let $\widetilde{G}$ be the subgroup of $G$ generated by $X$. Then there exists a locally definable subgroup $\breve{G} \leq \widetilde{G}$, a connected, finite-dimensional Lie group $U$, and a homomorphism $h: \breve{G} \rightarrow U$ as in Theorem 4.2, such that in addition, $U$ is Abelian.

Proof. Let $h, L, X_{n}$ be as in Corollary 4.13, For $k \in \mathbb{Z}$, for all $n \geq k$, define $X_{n}[k]=X_{n+k}$. This carries over to the ultraproduct, so $X[k]$ is defined for all $k \in \mathbb{Z}$, and has similar properties: $X[k] X[k] \subseteq X[k+1]$. Since the ultrafilter is nonprincipal, it concentrates on $n>k$, so by (5) of Corollary 4.13 we have $[X, X[k]] \subseteq X[k+n-1] \subseteq X\left[k^{\prime}\right]$ for all $k^{\prime} \in \mathbb{N}$. Factoring out $\bigcap_{k} h X[k]$ we obtain a commutative locally compact group. As in Theorem 4.2 we may replace it with a commutative Lie group.

We now deduce a version in the asymptotic setting. Here we do not obtain an infinite chain, but the function $f$ serves to say that the length of the chain is arbitrarily large compared to $e, c, k$. We say that two sets are $e$-commensurable if each is contained in the union of $\leq e$ cosets of the other. Taking $\nu$ to be the counting measure, we obtain (a strengthening of) Theorem 1.1.

Corollary 4.15. Let $f: \mathbb{N}^{2} \rightarrow \mathbb{N}$ be any function, and fix $k \in \mathbb{N}$. Then there exist $e^{*}, c^{*}, N \in \mathbb{N}$ such that the following holds.

Let $G$ be any group, $X$ a subset, and assume there exists a translation invariant finitely additive real-valued measure $\nu$ on the definable subsets of $G$ contained in some power of $X X^{-1}$, with $\nu\left(X X^{-1} X\right) \leq k \nu(X)$.

Then there exist $e \leq e^{*}, c \leq c^{*}$ and 0 -definable subsets $X_{N} \subseteq X_{N-1} \subseteq \cdots \subseteq X_{1}$, $N>f(e, c)$ such that $X^{-1} X$ and $X_{1}$ are e-commensurable and for $1 \leq m, n<N$ we have

(1) $X_{n}=X_{n}^{-1}$.

(2) $X_{n+1} X_{n+1} \subseteq X_{n}$.

(3) $X_{n}$ is contained in $\leq c$ translates of $X_{n+1}$.

(4) $a X_{n+1} a^{-1} \subseteq X_{n}$ for $a \in X_{1}$.

(5) $\left[X_{n}, X_{m}\right] \subseteq X_{k}$ whenever $k \leq N$ and $k<n+m$. In particular each $X_{n}$ is closed under the commutator bracket.

(6) $X_{n+1}=\left\{x \in X_{1}: x^{4} \in X_{n}\right\}$.

Proof. Fix $f, k$. We consider groups $G$ and subsets $X$ admitting a measure as above, with $\nu(X)=1, \nu\left(X X^{-1} X\right) \leq k$ (as we can always arrange by renormalizing $\nu)$. Consider integers $c$, and formulas $\phi$ of one free variable. Given $c, \phi$ and $X$, let $X_{1}$ be the subset defined by $\phi$. Let $N=f(e, c)+1$, and define $X_{n}$ using (6) for $2 \leq n \leq N$. Let us say that $(c, e, \phi)$ works for $X$ if properties (1)-(5) hold for the sets $X_{n}$ defined in this way.

We will show that for some finite set $\left(c_{1}, e_{1}, \phi_{1}\right), \ldots,\left(c_{n}, e_{n}, \phi_{n}\right)$, for any $G$ and any $k$-near-subgroup $X$ of $G$, some $\left(c_{i}, e_{i}, \phi_{i}\right)$ works for $X$. Suppose this is false. Then by the compactness theorem there exists $G$, a measure $\mu$ on the definable subsets of the group $\widetilde{G}$ generated by $X$ with $\mu(X)=1$, and a definable subset $X$ of $G$ with $\mu\left(X X^{-1} X\right) \leq k \mu(X)$ such that no $(c, \phi)$ works. But let $X_{1}$ be the definable set provided by Corollary 4.13, Let $c$ be the integer of Corollary 4.13 (3), and $e$ the 
number of translates of $X_{1}$ needed to cover $X$. Then $(c, e, \phi)$ works for $X$ (indeed the $X_{n}$ have the required properties beyond any bound). This contradiction proves the statement and the theorem.

Remark 4.16. (1) Again we can also add (7): if $x, y \in X_{2}$ and $x^{2}=y^{2}$, then $x y^{-1} \in X_{N}$.

(2) We could add that $X_{1} \subseteq\left(X^{-1} X\right)^{2}$ (as we do in the statement of Theorem 1.1), if we waive the 0 -definability of the $X_{n}$. They remain definable over parameters from the given structure. See Lemma 4.12

(3) This type of proof is always effective in the sense of Gödel. Note that if $f$ is recursive, then $e, c, N$ are automatically given by a recursive function (it suffices to search for $e, c, N, X_{1}$ such that (1)-(6) hold).

The sequence of subsets $X_{n}$ in Corollary 4.15 is recursively determined by $X_{1}$, via (6). The question is thus how to describe $X_{1}$. Studying the proof of the fundamental theorems on locally compact groups should provide detailed information; for now we state what is clear a posteriori when they are treated as a black box.

Regarding $X_{1}$, we have:

Corollary 4.17. Fix $k \in \mathbb{N}$, and $f$ as above. Then there exists $m$ and an algorithm that accepts as input the multiplication table of a finite near-subgroup $X$ up to $\left(X^{-1} X\right)^{m}$ and yields the set $X_{1}$ in polynomial time.

Proof. A formula in a logic with measure quantifiers $Q_{\epsilon}$ can be computed in polynomial time.

Note that we do not assume that $G$ itself is finite, and even if finite, it is not available to the algorithm, beyond $\left(X^{-1} X\right)^{m}$. Indeed the algorithm can be made to work for local groups.

We can improve the $m$ to 3 if, as in Remark 4.16 (2), we use a formula with a parameter from $X$. In this case the algorithm will first search for a parameter satisfying an appropriate auxiliary formula, then compute $X_{1}$ using this parameter.

To illustrate Theorem 4.12 we recover an easy version of a theorem of Freiman's (see [54]) (generalized to the noncommutative case).

Corollary 4.18. Fix $m, k$. Then there exists $e=e(k, m)$ with the following property. Let $G$ be a group of exponent $m$, i.e. $x^{m}=1$ for any $x \in G$. Let $X$ be a finite $k$-approximate subgroup of $G$. Then there exists a subgroup $S$ of $G$ such that $S, X$ are e-commensurable.

Proof. By compactness it suffices to show that if $X$ is a near-subgroup of a group $G$ of bounded exponent, then there exists a definable subgroup $S$ of $G$ such that $S, X$ are commensurable.

By a theorem of Schur's $(9,36.14)$, a periodic subgroup $P$ of $G L_{n}(\mathbb{C})$ has an abelian normal subgroup of finite index. When the period is bounded, the abelian subgroup and hence $P$ must be finite. If $L$ is a connected Lie group with center $Z$, by considering the action of $L$ on its Lie algebra we see that $L / Z$ is linear. Hence a periodic subgroup $P$ of $L$ of bounded period must be contained in $Z$ up to finite index, and again it follows that $P$ is finite.

Thus the image of $\breve{G}$ in the Lie group $L$ associated to $X$ is finite. Since this image is dense in $L$, and $L$ is connected, it follows that $L$ is trivial. The conclusion follows from Example 4.7 
Actually it is easy to see in the same way (using the fact that $L$ has no compact normal subgroups) that if $G$ is a periodic group and $X$ is a near-subgroup, then there exists a subgroup $S$ of $G$ such that $S, X$ are commensurable. This does not extend to families of finite approximate groups, since without a uniform bound taking an ultraproduct will not preserve periodicity.

\section{LiNEAR GROUPS}

Up to this point the hypotheses in this paper were purely measure-theoretic, at the top dimension as it were. We will now look at lower dimensions as well. Numerically this means that if a subset $Y$ of $X$ has about $c|X|^{\alpha}$ elements, we pay attention to $\alpha<1$ and not only to $c$ when $\alpha=1$. Our main tool is a cardinality estimate due in its original form to Larsen and Pink. In [24], it was presented as a dimension comparison lemma and slightly generalized in a number of directions; one of these will be needed here. We will first define quasi-finite dimension in general, and specifically for ultraproducts of finite approximate subgroups. Then, assuming the group is linear (or indeed densely embedded in a group with a nice dimension theory) we show that the ambient dimension dim constrains strongly the quasi-finite dimension. Finally, knowing that the group looks sufficiently noncommutative by certain measures using quasi-finite dimension, we can conclude using the stabilizer that it is in fact definable.

5.1. The semigroup of dimensions. Let $K$ be an ultraproduct of structures $K_{i}$ for some language $L$. For each $i$ we consider, along with $K_{i}$, the counting measure on definable sets, as a map from the class of definable sets into $\mathbb{R}$. Taking the ultraproduct of these maps as well, we obtain a map from the class of definable sets of $K$ into the ultrapower $\mathbb{R}^{*}$ of $\mathbb{R}$. This is a countably saturated real closed field. For nonempty definable $X$ (represented by a sequence $X\left(K_{i}\right)$ ), we have a nonstandard real number $\log |X|$ (represented by the sequence $\log \left|X\left(K_{i}\right)\right|$ ).

Let $C$ be a convex subgroup of $\mathbb{R}^{*}$. We assume $C$ is a countable union or a countable intersection of definable subsets of $\mathbb{R}^{*}$. Then $\mathbb{R}^{*} / C$ is an ordered $\mathbb{Q}$ vector space. We define $\delta(X)$ to be the image of $\log |X|$ in $\mathbb{R}^{*} / C$. We view $\delta$ as a (nonintegral) dimension.

Subadditivity: Let $f: X \rightarrow Y$ be a definable map; assume $\delta\left(f^{-1}(y)\right) \leq \alpha=$ $a+C$ for each $y \in Y$, and $\delta(Y) \leq \beta=b+C$. Then $\delta(X) \leq \alpha+\beta$. To see this, if $C=\bigcup_{n} C_{n}$ is a countable union, we may take $C_{1} \subset C_{2} \subset \ldots$ We have $\log |Y| \leq b+c$ with $c \in C_{n}$ for some $n$, and by compactness, $\log \left|f^{-1}(y)\right| \leq a+c^{\prime}$, with $c^{\prime} \in C_{n^{\prime}}$ for some $n^{\prime}$. It follows that $\log |X| \leq a+b+c+c^{\prime}$, so $\delta(X) \leq \alpha+\beta$. If $C=\bigcap_{n} C_{n}$, then $\log |Y|-b \in C_{n}$, and $\log \left|f^{-1}(y)\right|-a \in C_{n}$ for each $n$; hence $\log |X|-a-b \in C_{n}$ for each $n$.

We would like to extend the dimension to $\bigwedge$-definable sets. Fix $\delta_{0} \in \mathbb{R}^{*}, \delta_{0}>C$. Let $V_{0}=V_{0}\left(\delta_{0}\right)$ be the group of elements $a \in \mathbb{R}^{*} / C$ such that $-n \delta_{0}+C \leq a \leq$ $n \delta_{0}+C$ for some $n \in \mathbb{N}$. Let $V=V\left(\delta_{0}\right)$ be the set of cuts of $V_{0}$, i.e. subsets $s \subset V_{0}$ that are nonempty, bounded above, and closed downwards. This is a semigroup under set addition, linearly ordered by set inclusion. $V_{0}$ embeds into $V$, by $a \mapsto\{v: v \leq a\}$. We identify $V_{0}$ with its image in $V$. Any subset of $V$ that is bounded below has a greatest lower bound, namely the intersection. We note that $V_{0}$ consists of invertible elements of $V$, and that it is semi-dense in $V$, in the sense that if $u<v \in V$, then there exists $z \in V_{0}$ with $u<z \leq v$. 
It will suffice for our purposes to use the intermediate subsemigroup $V_{1}$ consisting of infima of bounded countable subsets of $V_{0}$.

We could also form the linearly ordered semigroup $V^{\prime}$ of cuts in $V_{0}^{\prime}:=\left\{a \in \mathbb{R}^{*}\right.$ : for some $\left.n \in \mathbb{N}, \quad-n \delta_{0} \leq a \leq n \delta_{0}\right\}$. The natural map $V_{0}^{\prime} \rightarrow V_{0}$ maps cuts to cuts and respects addition and $\leq . V$ can be identified with the ordered subsemigroup of cuts $I \in V^{\prime}$ with $C+I=I$. Note that for a subset of $V$, the infimum (in the sense of $V^{\prime}$ ) lies in $V$ and agrees with the infimum in $V$.

When $\alpha_{n}, \beta_{n}$ are descending sequences of cuts, $\inf _{n}\left(\alpha_{n}+\beta_{n}\right)=\inf _{n} \alpha_{n}+\inf _{n} \beta_{n}$ holds in $V$. By the above remark, it suffices to check this in $V^{\prime}$. The inequality $\geq$ is clear, since $\alpha_{n}+\beta_{n} \geq \inf _{n} \alpha_{n}+\inf _{n} \beta_{n}$ for each $n$. For the other inequality, let $\alpha_{n}^{\prime} \in \alpha_{n} \backslash \alpha_{n+1}, \beta_{n}^{\prime} \in \beta_{n} \backslash \beta_{n+1}$. Suppose $c \in \mathbb{R}^{*}$ and $c \leq \inf _{n}\left(\alpha_{n}+\beta_{n}\right)$. Then by countable saturation there exist $(\alpha, \beta) \in \mathbb{R}^{*}$ with $\alpha \leq \alpha_{n}^{\prime}, \beta \leq \beta_{n}^{\prime}$ for each $n$, and $c \leq \alpha+\beta$. Hence $c \leq \inf _{n} \alpha_{n}+\inf _{n} \beta_{n}$.

Let us also point out that if $\alpha<\alpha^{\prime}$ and $\beta<\beta^{\prime}$ are cuts, then $\alpha+\beta<\alpha^{\prime}+\beta^{\prime}$. This holds for any semigroup of cuts in a dense linearly ordered group; to prove it we may consider the semigroup of all cuts. Let $a \in \alpha^{\prime} \backslash \alpha, b \in \beta^{\prime} \backslash b$. Let $a^{-}=\{x: x<a\}$, and similarly for $b^{-}$. We have $a^{-}+b^{-} \leq a+b$, and $a^{-}+b^{-} \neq a+b$ since the cut $a^{-}+b^{-}$has no maximal point. Thus $\alpha+\beta \leq a^{-}+b^{-}<a+b \leq \alpha^{\prime}+\beta^{\prime}$. (One strict inequality and one weak inequality would not suffice for the same.)

The multiplicative group $\mathbb{Q}^{>0}$ acts on $V_{0}$, and hence on $V$.

\subsection{Quasi-finite dimension. For an $\bigwedge$-definable set $X$ define:}

$$
\delta(X)=\inf \delta(D),
$$

where $D$ ranges over all definable sets containing $X$. Note a continuity property of the dimension: If $X=\bigcap X_{n}$ with $X_{1} \supset X_{2} \supset \ldots \wedge$-definable, then $\delta(X)=$ $\inf _{n} \delta\left(X_{n}\right)$.

The subadditivity property holds for $\Lambda$-definable sets $X$ : let $f$ be a definable map, let $\gamma \in V_{1}$, and assume $\delta\left(f^{-1}(a) \cap X\right) \leq \gamma$ for all $a$. Then $\delta(X) \leq \delta(f(X))+\gamma$. Indeed if $X=\bigcap X_{n}$ with $X_{n}$ a descending sequence of definable sets, then $f(X)=$ $\bigcap_{n} f\left(X_{n}\right)$ by compactness (saturation); say $\gamma=\inf \gamma_{k}$; then for each $k$, for some $n(k)$, we have $\delta\left(f^{-1}(a) \cap X_{n(k)}\right) \leq \gamma_{k}$, again by compactness. So $\delta\left(X_{n(k)}\right) \leq$ $\delta\left(f\left(X_{n(k)}\right)\right)+\gamma_{k}$. Thus $\inf _{n} \delta\left(X_{n}\right) \leq \inf _{k} \delta\left(X_{n(k)}\right) \leq \inf _{k} \delta\left(f\left(X_{n(k)}\right)\right)+\inf _{k} \gamma_{k}=$ $\delta(f(X))+\gamma$.

As a very special case of subadditivity, noting that $\delta(F)=0$ for finite $F$, we have $\delta\left(D_{1} \cup D_{2}\right)=\max \left(\delta\left(D_{1}\right), \delta\left(D_{2}\right)\right)$.

Also, $\delta\left(D_{1} \times D_{2}\right)=\delta\left(D_{1}\right)+\delta\left(D_{2}\right)$ : let $\mathcal{E}_{i}$ be the family of definable sets containing $D_{i}$. For any definable set $E$ with $D_{1} \times D_{2} \subseteq E$ there exist (by compactness) $E_{i} \in \mathcal{E}_{i}$ with $D_{i} \subseteq E_{i}(i=1,2)$ and $E_{1} \times E_{2} \subseteq E$. Thus $\delta\left(D_{1} \times D_{2}\right)=\inf _{E_{1} \in \mathcal{E}_{1}, E_{2} \in \mathcal{E}_{2}} \delta\left(E_{1} \times\right.$ $\left.E_{2}\right)=\inf _{E_{1}, E_{2}}\left(\delta\left(E_{1}\right)+\delta\left(E_{2}\right)\right)=\inf _{E_{1}} \delta\left(E_{1}\right)+\inf _{E_{2}} \delta\left(E_{2}\right)=\delta\left(D_{1}\right)+\delta\left(D_{2}\right)$.

If $X$ is $\Lambda$-definable over a set $A$, there exists a complete type $P$ over $A$ containing $X$ with $\delta(X)=\delta(P)$. To see this it suffices to check that $X \backslash \bigcup\{D: \delta(D)<\delta(X)\}$ is nonempty, since any type extending this will do. By compactness it suffices to see that $X$ is not contained in a finite union of sets $D$ with $\delta(D)<\delta(X)$. This is clear using $\delta\left(D_{1} \cup D_{2}\right)=\max \left(\delta\left(D_{1}\right), \delta\left(D_{2}\right)\right)$.

If $\delta(X) \in V_{0}$, we say that $X$ has strict quasi-finite dimension. Note in this case, by saturation of $\mathbb{R}^{*}$, that if $\delta(X)=\inf _{n \in \mathbb{N}} \alpha_{n}$, then $\delta(X)=\alpha_{n}$ for large enough $n$.

5.3. Examples. The best behaved case is of totally categorical theories, i.e. theories $T$ with a unique model in each power; a basic example is the theory of vector 
spaces over a fixed finite field. In this case, any finite subset $T_{0}$ of $T$ has finite models, described by a single integer parameter; the cardinality is precisely given by a polynomial $P$ in this dimension parameter. In this case, regardless of the choice of convex subgroup, the quasi-finite dimension equals the degree of the Zilber polynomial (times a fixed scalar) and recovers the Morley dimension. This was an essential ingredient of Zilber's theory of totally categorical structures; see [8] for generalizations and converses.

In general, the two most natural choices for a convex subgroup $C$ are the smallest nontrivial convex subgroup, the convex hull $C_{\min }$ of $\mathbb{Z}$, and the largest convex subgroup $C_{\max }$ with $\delta_{0} \notin C_{\max }$.

$C_{\text {max }}$ is a countable intersection of definable subsets of $\mathbb{R}^{*}$. The corresponding group of dimensions is canonically isomorphic to $\mathbb{R}$ (with $\delta_{0}$ mapping to 1 ). Each dimension $\alpha$ induces an ideal $I_{\alpha}$ (Example 2.13), which is not S1. Asymptotically, a set $X$ represented by a sequence of finite sets $X_{i}$ has the same dimension as $Y \subset X$ (represented by $Y_{i}$ ) if for any $\epsilon$, for almost all $i,\left|Y_{i}\right| \geq\left|X_{i}\right|^{1-\epsilon}$.

$C_{\min }$ is a countable union of definable subsets of $\mathbb{R}^{*}$. The group of dimensions is more complicated, but when $\delta(X)=\alpha$, the ideal of lower-dimensional subsets of $X$ is an S1-ideal. Here $Y \subset X$ has the same dimension as $X$ if for some $k,\left|X_{i}\right| \leq k\left|Y_{i}\right|$ for almost all $i$.

5.4. Minimality. Now assume that each $K_{i}$ is a field, possibly with additional structure. There will be no loss of generality in assuming that $K_{i}$ is algebraically closed. Let $K$ be an ultraproduct of the structures $K_{i}$. Constructible sets and varieties will be assumed to be defined over $K$. Here the word "constructible" means: definable in $K=K^{\text {alg }}$ as a field, whereas "definable" means: definable in $(K, \cdots)$ as an $L$-structure. For a constructible set $S$, aside from the pseudo-finite dimension $\delta$ constructed above, we have the dimension in the sense of algebraic varieties. This can be defined as the Morley rank of the set, viewed as definable in $(K,+, \cdot)$, or it can be defined as the dimension of the Zariski closure of $S$, as in [56]; see [4].

Let $G$ be a simple algebraic group over the ultraproduct $K$. $G$ can be viewed as a group subvariety of the group $G L_{n}$ of invertible matrices. We write $G$ when we think of the defining equations, and $G(K)$ when we think of the set of points of $K$.

Let $\Gamma_{0}$ be a Zariski dense subset of $G(K)$. Consider the functions $F_{c}(x, y)=$ $c x^{-1} c^{-1} y, c \in \Gamma_{0}$. Any subvariety $H$ of $G(K)$ closed under all the $F_{c}$ must be a group subvariety of $G$, normalized by $\Gamma_{0}$, hence by the Zariski closure of this group, i.e. by $G$. Since $G$ is simple, we must have $H=1$ or $H=G$.

It follows that if $Y, Z$ are constructible subsets of $G$, defined over a subfield $A$ of $K$, and if $0<\operatorname{dim}(Y) \leq \operatorname{dim}(Z)<\operatorname{dim}(G)$, then $\operatorname{dim}\left(F_{c}(Y \times Z)\right)>\operatorname{dim}(Z)$ for some $c \in \Gamma_{0}$. Moreover, let $Y \times^{\prime} Z=Y \times Z \backslash \bigcup_{j} W_{j}$, where $W_{j}$ ranges over all $A$-definable constructible subsets $W$ of $Y \times Z$ with $\operatorname{dim}(W)<\operatorname{dim}(Y)+\operatorname{dim}(Z)$. (This is the same, for the theory ACF of algebraically closed fields, as the product $\times_{n f}$ encountered in the proof of Theorem 3.5. Note that this product depends on the base set $A$, but as $A$ will be fixed we will omit it from the notation.) Then $\operatorname{dim}\left(F_{c}\left(Y \times^{\prime} Z\right)\right)>\operatorname{dim}(Z)$ for some $c$. This is a typical application of Zilber's stabilizer, and in itself an instance of the "sum-product" phenomenon in a constructible setting: we may assume that $Z$ is irreducible. If $\operatorname{dim}\left(F_{c}\left(Y \times^{\prime} Z\right)\right)=$ $\operatorname{dim}(Z)$, we find that $Y$ and all $\Gamma_{0}$-conjugates of $Y$ are contained in finitely many cosets of the Zilber stabilizer $H=\{y: \operatorname{dim}(y Z \triangle Z)<\operatorname{dim}(Z)\}$, up to smaller 
dimension. But then $\bigcap_{x \in \Gamma_{0}} x^{-1} H x$ (a finite intersection of conjugates of $H$ ) is closed under all the $F_{c}$, so by the first paragraph it equals $G$, i.e. contradicts the previous paragraph.

This property of $\left(G, F_{c}\right)_{c \in \Gamma_{0}}$ is referred to as minimality. See [24], Example 2 for details and generalizations.

5.5. The dimension inequality. Let $\Gamma \subseteq X$ be an $\bigwedge$-definable subgroup, of strict quasi-finite dimension $\delta(\Gamma)=\delta(X)$; and with $\Gamma_{0} \leq \Gamma$. Let $\delta_{0}=\delta(\Gamma), V=V\left(\delta_{0}\right)$. For $n \in \mathbb{Q}$ and $v \in V, n v$ is defined; we write $\gamma_{0} n$ for $n \gamma_{0}$. Let $\gamma_{0}=\delta(\Gamma) / \operatorname{dim}(G)$.

For a constructible $Z \subseteq G(K)^{n}$, define $\delta_{\Gamma}(Z)=\delta\left(Z \cap \Gamma^{n}\right)$. For any $W \subset G(K)^{n}$, let $\operatorname{dim}(W)$ denote the dimension of the Zariski closure of $W$.

Proposition 5.6. For any constructible $Z \subseteq G^{n}$, we have $\delta_{\Gamma}(Z) \leq \gamma_{0} \operatorname{dim}(Z)$.

Proof. This is a special case of Corollary 1.12 of [24, as generalized in Remark 1.11. We give the proof in the present case, for $Z \subseteq G$. For $G^{n}$, see the remark below.

Let $W$ be an $\Lambda$-definable subset (over $A$ ) of $\Gamma^{n}$. Call $W$ unbalanced if $\delta(W)>$ $\gamma_{0} \operatorname{dim}(W)$. There exists a complete type $W^{\prime} \subset W$ defined over $A$ with $\delta_{\Gamma}\left(W^{\prime}\right)=$ $\delta_{\Gamma}(W)$. As $\operatorname{dim}\left(W^{\prime}\right) \leq \operatorname{dim}(W), W^{\prime}$ is unbalanced if $W$ is.

We must show that no unbalanced sets exist. Otherwise, let $Y, Z$ be unbalanced $\bigwedge$-definable sets with $\operatorname{dim}(Y)$ minimal, and $\operatorname{dim}(Z)$ maximal possible. Clearly $0<\operatorname{dim}(Y) \leq \operatorname{dim}(Z)<\operatorname{dim}(G)$. Say $Y, Z, c$ are defined over the countable $A \leq K$. By the above, we may take $Y, Z$ to be complete types over $A$. Form $Y \times^{\prime} Z$. By minimality of $\left(G, F_{c}\right)_{c \in X}$ there exists $c \in X$ with $\operatorname{dim}\left(F_{c}\left(Y \times^{\prime} Z\right)\right)>\operatorname{dim}(Z)$.

We note first that $Y \times^{\prime} Z$ is balanced: Let $f$ be the restriction of $F_{c}$ to $Y \times^{\prime} Z$. Then since $Y \times^{\prime} Z$ implies a complete quantifier-free type over $A$ in the language of fields, the fiber dimension $\operatorname{dim} f^{-1}(a)$ is constant $(=b)$ for $a \in F_{c}\left(Y \times^{\prime} Z\right)$, and from $\operatorname{dim}\left(f\left(Y \times^{\prime} Z\right)\right)>\operatorname{dim}(Z)$ it follows that $b<\operatorname{dim}(Y)$. So the fibers are not unbalanced, i.e. $\delta_{\Gamma}\left(f^{-1}(a)\right) \leq b \gamma_{0}$. On the other hand since $\operatorname{dim}\left(f\left(Y \times^{\prime} Z\right)\right)>$ $\operatorname{dim}(Z), f\left(Y \times^{\prime} Z\right)$ is not unbalanced either, so $\delta_{\Gamma}\left(f\left(Y \times^{\prime} Z\right)\right) \leq \operatorname{dim}\left(f\left(Y \times^{\prime} Z\right)\right) \gamma_{0}$. By subadditivity we obtain $\delta_{\Gamma}\left(Y \times^{\prime} Z\right) \leq\left(b+\operatorname{dim}\left(f\left(Y \times^{\prime} Z\right)\right)\right) \gamma_{0}=\operatorname{dim}\left(Y \times^{\prime} Z\right) \gamma_{0}$.

Now there exists a complete type $Q$ over $A$ with $Q \subseteq Y \times Z$ and $\delta_{\Gamma}(Q)=$ $\delta_{\Gamma}(Y)+\delta_{\Gamma}(Z)$. I claim that $Q=Y \times^{\prime} Z$ (formed over $A$ ). For if $Q$ is any other type, the fibers $Q_{a}=\{w:(w, a) \in Q\}$ have dimension $\operatorname{dim}\left(Q_{a}\right)=b^{\prime}<\operatorname{dim}(Y)$ for $a \in Z$ (the dimension is constant on $Z$ since $Z$ is a complete type). By minimality of $\operatorname{dim}(Y)$ we have $\delta_{\Gamma}\left(Q_{a}\right) \leq b^{\prime} \gamma_{0}$. By subadditivity it follows that

$$
\delta_{\Gamma}(Y)+\delta_{\Gamma}(Z)=\delta_{\Gamma}(Q) \leq b^{\prime} \gamma_{0}+\delta_{\Gamma}(Z)<\operatorname{dim}(Y) \gamma_{0}+\delta_{\Gamma}(Z) \leq \delta_{\Gamma}(Y)+\delta_{\Gamma}(Z) .
$$

So $Q=Y \times^{\prime} Z$.

Since $\gamma_{0} \operatorname{dim}(Y)<\delta_{\Gamma}(Y)$ and $\gamma_{0} \operatorname{dim}(Z)<\delta_{\Gamma}(Z)$, we have $\gamma_{0} \operatorname{dim}\left(Y \times^{\prime} Z\right)=$ $\gamma_{0}(\operatorname{dim}(Y)+\operatorname{dim}(Z))<\delta_{\Gamma}(Y)+\delta_{\Gamma}(Z)=\delta_{\Gamma}\left(Y^{\prime} \times Z\right)$. So $Y \times^{\prime} Z$ is unbalanced, a contradiction.

Remark 5.7. Let $f: X \rightarrow X^{\prime}$ be a constructible map, $\Gamma$ an $\bigwedge$-definable subset of $X, \Gamma^{\prime}=f(\Gamma)$. If the inequality of Proposition 5.6 holds for $\Gamma^{\prime} \subseteq X^{\prime}$ and for each fiber $f^{-1}(a) \subseteq X, a \in \Gamma^{\prime}$, all with the same value of $\gamma_{0}$, then it holds for $\Gamma \subseteq X$. This is an easy consequence of subadditivity and definability of Zariski dimension; cf. [24.

Recall that a morphism $f: U \rightarrow V$ of algebraic varieties is dominant if there exists no proper subvariety $V^{\prime}$ of $V$ such that the image of $U$, over any field, is contained in $V^{\prime}$. 
Lemma 5.8. Let $U$ be a Zariski open subset of $G^{m}$. Let $f: U \rightarrow W \subseteq G^{n}$ be a dominant morphism of varieties. Then $\delta\left(f\left(U \cap \Gamma^{m}\right)\right)=\operatorname{dim}(W) \gamma_{0}$.

Proof. We first show that if $U$ is Zariski open in $G^{m}$, then $\delta_{\Gamma}(U)=m \operatorname{dim}(G) \gamma_{0}$. We have $\delta\left(\Gamma^{m}\right)=m \delta_{\Gamma}(G)$, On the other hand, if $V$ is a proper Zariski closed subset of $\Gamma^{m}$, then $\operatorname{dim}(V) \leq m \operatorname{dim}(G)-1$, and by Proposition 5.6. $\delta\left(V \cap \Gamma^{n}\right) \leq$ $\operatorname{dim}(V) \gamma_{0}<m \delta_{\Gamma}(G)$. It follows that $\delta\left(\Gamma^{m} \backslash V\right)=m \delta_{\Gamma}(G)=m \operatorname{dim}(G) \gamma_{0}$.

There exists a relatively Zariski open $W^{\prime} \subseteq W$, $\operatorname{dim}\left(W^{\prime}\right)=\operatorname{dim}(W)$, such that $\operatorname{dim} f^{-1}(b)$ is constant for $b \in W^{\prime}$. Replacing $W$ by $W^{\prime}$ and $U$ by $f^{-1}\left(W^{\prime}\right)$, we may assume that $\operatorname{dim} f^{-1}(b)=d$ is constant for $b \in W$. So $\operatorname{dim}(U)=d+\operatorname{dim}(W)$. By Proposition [5.6] for any $b \in W \cap \Gamma^{n}$, we have $\delta_{\Gamma}\left(f^{-1}(b)\right) \leq d \gamma_{0}$. Hence if $\delta\left(f\left(U \cap \Gamma^{m}\right)\right)=\gamma<\operatorname{dim}(W) \gamma_{0}$, then by subadditivity of $\delta$ we have $\delta\left(U \cap \Gamma^{m}\right) \leq$ $\gamma+d \gamma_{0}<(\operatorname{dim}(W)+d) \gamma_{0}=\operatorname{dim}(U) \gamma_{0}$; this contradicts the first paragraph. Note that adding the invertible element $d \gamma_{0}$ preserves strict inequalities.

In case $f\left(U \cap \Gamma^{m}\right) \subseteq \Gamma^{n}$, it follows that $\delta_{\Gamma}(W)=\operatorname{dim}(W) \gamma_{0}$. The proof shows more generally that the class of subvarieties $U$ of $G^{m}$ satisfying $\delta_{\Gamma}\left(U^{\prime}\right)=\operatorname{dim}(U) \gamma_{0}$ for all Zariski dense open $U^{\prime}$ is closed under forward images of such morphisms.

Note that an $\bigwedge$-definable subgroup $\Gamma$ of $G(K)$ has strict quasi-finite dimension iff for some $\bigvee$-definable $\widetilde{G}$ containing $\Gamma, \widetilde{G} / \Gamma$ is bounded.

5.9. From now on we assume $C$ is the convex hull of $\mathbb{R}$ in $\mathbb{R}^{*}$, a $\bigvee$ definable convex subgroup. For $Y \subseteq X$, let $\mu(Y)$ be the unique real number $r$ such that for any rational $\alpha, \alpha|X|>|Y|$ if $\alpha>r$ and $\alpha|X|<|Y|$ if $\alpha<r$. Then $\mu$ is a definable measure on definable subsets of $X$, and we have $\mu(Y)>0$ iff $\delta(Y)=\delta(\Gamma)=\delta(X)$.

Proposition 5.10. Let $\Gamma$ be a Zariski dense $\bigwedge$-definable subgroup of $G(K), G$ a semisimple algebraic group over $K$. Assume $\Gamma$ has strict quasi-finite dimension. Then $\Gamma$ is definable.

Proof. Assume first that $G$ is simple. Let $K^{a}$ be the algebraic closure of $K$. Let $\Gamma_{0} \leq \Gamma$ be any Zariski dense set of points, so that the previous lemmas apply. Since $G$ is a simple group, any noncentral conjugacy class $C$ of $G\left(K^{a}\right)$ generates $G$ in a finite number $d \leq 2 \operatorname{dim}(G)$ of steps. Thus for any noncentral $b$, the morphism of varieties $f_{b}: G^{d} \rightarrow G, f\left(x_{1}, \ldots, x_{d}, b\right)=x_{1}^{-1} b x_{1} x_{2}^{-1} b x_{2} \cdots x_{d}^{-1} b x_{d}$ is surjective on $K^{a}$-points. By Lemma [5.8, $\delta\left(f_{b}\left(\Gamma^{d}\right)\right) \geq \operatorname{dim}(G) \gamma_{0}=\delta(G)$. Let $X$ be a definable set containing $\Gamma$ with $\delta(X)=\delta(\Gamma)$, and let $\widetilde{G}$ be the group generated by $X$. Then $\widetilde{G} / \Gamma$ is bounded. Let $S \subseteq \Gamma$ be an $\bigwedge$-definable normal subgroup of $\widetilde{G}$ with $\widetilde{G} / S$ bounded (Lemma 3.4), and choose a noncentral $b \in S$. Let $Y$ be the definable set $Y=f_{b}\left(X^{d}\right)$. Since $S$ is normal, $Y \subseteq S$. We have $\delta(Y) \geq \delta\left(f_{b}\left(\Gamma^{d}\right)\right)=\delta(G)$, so $\mu(Y)>0$. Hence $S$ contains a bounded finite number of disjoint translates $s_{i} Y$ of $Y$, so any $s \in S$ lies in $s_{i} Y Y^{-1}$ for some $i$ (Ruzsa's argument). Hence $S=\bigcup_{i} Y Y^{-1}$ is definable. Since $\Gamma / S$ is bounded and $\bigwedge$-definable, it must be finite, so $\Gamma$ is definable too.

When $G$ is semisimple, we proceed by induction on $\operatorname{dim}(G)$. Let $N$ be a normal algebraic subgroup, $\pi: G \rightarrow G / N$ the natural homomorphism. Since $\Gamma$ has strict quasi-finite dimension, for some $\bigvee$-definable $\widetilde{G}$ we have that $\widetilde{G} / \Gamma$ is bounded. It follows that $(N \cap \widetilde{G}) /(N \cap \Gamma)$ and $\pi(\widetilde{G}) / \pi(\Gamma)$ are bounded, so $N \cap \Gamma$ and $\pi(\Gamma)$ have strict quasi-finite dimension; by induction they are definable. By Remark $4.10, \Gamma$ is definable. 
Proof of Theorem 1.3, and Corollary. Suppose not. Then there exists an ultraproduct $(K, X)$ of $\left(K_{i}, X_{i}\right)$ such that for no definable subgroup $H$ of $G(K)$ do we have $H \subseteq\left(X^{-1} X\right)^{2}$ and $X$ contained in finitely many cosets of $H$. However $X$ is a near-subgroup of $G(K)$. Let $\widetilde{G}$ be the subgroup generated by $X$. By Theorem 3.5 there exists an $\bigwedge$-definable group $\Gamma \subseteq\left(X^{-1} X\right)^{2}$, normal in $\widetilde{G}$, with $X / \Gamma$ bounded. By Proposition 5.10, $\Gamma$ is definable. By compactness, $X / \Gamma$ is finite. This contradiction proves the theorem.

The corollary easily follows, and can also be quickly proved directly in the same way: if it fails, we obtain an ultraproduct $(K, X)$ with $X$ an infinite near-subgroup, generating a $\bigvee$-definable group $\widetilde{G}$ strictly bigger than $\left(X^{-1} X\right)^{2}$, and such that no infinite definable proper subgroup of $\widetilde{G}$ is normalized by $X$. Let $\Gamma$ be as above. Again $\Gamma$ is definable; hence (being normalized by $X$ ) either $\Gamma=\widetilde{G}$ or $\Gamma$ is finite. If $\Gamma$ is finite, then since $X / \Gamma$ is bounded it is finite, contradicting the assumption that $X$ is infinite. If $\Gamma=\widetilde{G}$, then since $\Gamma \subseteq\left(X^{-1} X\right)^{2}$ we must have $\widetilde{G}=\left(X^{-1} X\right)^{2}$, again a contradiction.

Similarly we can obtain $|S| /\left|X^{-1} X\right| \geq .9$ in Corollary 1.4, otherwise we obtain $(K, X)$ as above and also a measure $\mu$ on $\widetilde{G}$ with no infinite definable subgroup $H$, contained in $\widetilde{G}$ and normalized by $X$, satisfying $\mu(H) / \mu\left(X^{-1} X\right) \geq .99$. But again $\Gamma$ is definable, and by Theorem 3.5, $\Gamma \backslash X^{-1} X$ is contained in a union of non- $\mu$ wide sets; by saturation and definability of $\Gamma$ it is contained in finitely many such sets, so $\mu\left(\Gamma \backslash X^{-1} X\right)=0$, a contradiction. One can also get $X_{i} X_{i}^{-1} X_{i}=S_{i}$ from the fact that $q q^{-1} q$ is a coset of $S$ in Theorem 3.5. and that $S_{i}$ has no subgroups of bounded index. I noted this stronger statement after László Pyber pointed out that the statement of Corollary 1.4 implies $X_{i} X_{i}^{-1} X_{i}=S_{i}$, using [40].

One can immediately deduce a version for arbitrary linear groups:

Corollary 5.11. Let $k, n \in \mathbb{N}$. Then there exist $k^{\prime} \in \mathbb{N}$, such that if $X$ is a $k$-approximate subgroup of $G L_{n}(K)$ for some field $K$, then there exist algebraic subgroups $H \leq G$ of $G L_{n}$ with $H$ solvable and normal in $G$, and a subgroup $\Delta$ of $G$ (normalized by $X$ ) with $\Delta \subseteq\left(X^{-1} X\right)^{2} H$ and such that $X$ is contained in $\leq k^{\prime}$ cosets of $\Delta$.

The groups $H, N$ are defined by polynomial equations in the matrix entries; these equations can be taken to have degree bounded by a function of $k, n$ alone.

Jordan has shown that finite subgroups of linear groups are bounded, up to an Abelian part, provided they contain no nontrivial unipotent elements. (Jordan's beautiful proof occupies some 13 pages of [29. 9] contains a different proof in characteristic 0, due to Frobenius.) We may now extend this to say that approximate subgroups of connected Lie groups are bounded, up to a (connected, closed) solvable subgroup.

Corollary 5.12. Let $k \in \mathbb{N}$, and let $L$ be a connected Lie group of dimension $d$. Then there exist $k^{\prime \prime} \in \mathbb{N}$, such that if $X$ is a (finite) $k$-approximate subgroup of $L$, then there exists a $(d+2)$-solvable subgroup $S$ of $L$ such that $X$ is contained in $\leq k^{\prime \prime}$ cosets of $S$.

Proof. Let $X$ be a $k$-approximate subgroup of $L$. Assume first that $L$ embeds into $G L_{d}(\mathbb{R})$. In this case, let $H, G$ be the subgroups provided by Corollary 5.11 so $H$ is $d$-solvable. So $X$ is contained in boundedly many cosets of a subgroup $\Delta$ of 
$G / H$, with $\Delta / H$ finite (as $X$ is finite). By [29, $\Delta / H$ contains a normal Abelian subgroup $S / H$ of bounded index. Then $S$ is $(d+1)$-solvable, and $X$ is contained in boundedly many cosets of $S$.

In general, let $Z$ be the center of $L$. Then $L / Z$ acts faithfully on the Lie algebra of $L$ by conjugation, so it embeds into $G L_{d}(\mathbb{R})$. By the linear case, if $X$ is a $k$-approximate subgroup of $L$, then the image of $X$ in $L / Z$ is a $k$-approximate subgroup of $L / Z$, so by the linear case it is contained in boundedly many cosets of a solvable subgroup $S / Z$. The pullback $S$ of $S / Z$ to $L$ is $(d+2)$-solvable, and finitely many cosets of $S$ cover $X$.

\section{Remarks.}

(1) Once the definability of $\Gamma$ is established, it is known to be definable in the field language, possibly expanded by an automorphism, and indeed to be a simple group of (possibly twisted) Lie type; see [35.

(2) We could also deduce Theorem 1.3 from Corollary 1.2 the proof of Proposition 5.10, together with saturation, shows that for some $m \in \mathbb{N}$ we have $\mu\left(C_{b}^{d}\right) \geq 1 / m$ for all noncentral $b$. It follows that with probability very close to 1 (in $b$ ), $\mu\left(C_{b}^{d}\right) \geq 1 / m$; so the hypothesis of Corollary 1.2 holds.

(3) Using another direction of generalization taken in 24], results of this section are valid for near-subgroups of groups $G$ of finite Morley rank, in place of algebraic groups.

We further remark that Theorem 1.1 of [5] in the sum-product setting, as well as the theorem of [20] for subsets of $S L_{2}\left(\mathbb{F}_{p}\right)$, can be put in the framework of Proposition 5.10 if one takes the $C=C_{\max }$ to be the largest convex subgroup of $\mathbb{R}^{*}$ not containing $\delta_{0}$ (in place of the smallest nonzero convex subgroup, as we took it to be) 2

\section{UNIFORM DEFINABILITY OF THE TOPOLOGY}

We prove a stronger version of the stabilizer theorem for arbitrary S1-ideals on an Ind-definable group, with more uniform control of the topology of the Lie group. It follows that the Lie group associated to a near-subgroup is always associated already to the reduct to a finite sublanguage. Stronger uniformity statements in this direction may give a more powerful means for finitization of results about saturated models.

Remark 6.1. Let $T$ be a simple theory, or a NIP theory. Then the forking ideal is an S1-ideal.

Proof. Let $\left(a_{i}\right)$ be an $A$-indiscernible sequence, and suppose $\phi\left(x, a_{i}\right)$ does not fork over $A$. We have to show that $\phi\left(x, a_{i}\right) \wedge \phi\left(x, a_{j}\right)$ does not fork over $A$, for some $i \neq j$.

Simple case: the $a_{i}$ are independent over some $M$. Let $c_{i}$ be such that $\phi\left(c_{i}, a_{i}\right)$ with $c_{i}, a_{i}$ independent; choose $c_{i}$ so that $c_{i}, M$ are independent over $a_{i}$. Then $c_{i}, M a_{i}$ are independent over $A$. The sequence $\left(a_{i}\right)$ could be taken to be long; by refining it we can assume that $t p\left(a_{i} / M\right)$ is constant. By 3 -amalgamation we can find $c$ independent over $M$ from $\left(a_{i}\right)_{i}$, with $t p\left(c, a_{i} / M\right)=t p\left(c_{i}, a_{i} / M\right)$. Since

\footnotetext{
${ }^{2}$ In fact, after these lines were written, Breuillard, Green and Tao essentially took this route; using a beautiful analysis of the geometry of tori, continuing a line started in 29], they obtain an effective, polynomial version of Theorem 1.3 See 3 .
} 
$t p\left(c_{i} / M\right)=t p(c / M), c, M$ are independent over $A$, so $c$ is independent from $a_{1}, a_{2}$ over $A$. Hence $t p\left(c^{\prime} / a_{1} a_{2}\right)$ does not fork over $A$.

NIP case: Let $q_{i}$ be a global type with $\phi\left(x, a_{i}\right) \in q_{i}$, such that $q_{i}$ does not fork over $A$. Let $M$ be a model containing $A$. Then $q_{i}$ does not fork over $M$. So $q_{i}$ is $M$-invariant. There are few choices for $M$-invariant types, so $q_{i}=q_{j}$ for some $i \neq j$. Since $q_{i}$ does not fork over $A, \phi\left(x, a_{i}\right) \wedge \phi\left(x, a_{j}\right)$ does not fork over $A$.

Let $X$ be a topological space, $p \in X$. We say that a collection $C$ of sets strongly generates the topology at $p$ if $p$ is in the interior of each set in $C$, and any open neighborhood of $p$ contains some element of $C$.

Let $M$ be a Riemannian manifold. Let $\rho(p, q)$ denote the Riemannian distance, and $B(p, r)$ (respectively $\bar{B}(p, r))$ the open (resp. closed) ball of radius $r$. A geodesic ball around $p$ is the image under the exponential map exp of a ball $b$ of radius $r$ around 0 in the tangent space to $p$, where $r$ is small enough that $\exp _{p}$ is a diffeomorphism. We have $\rho\left(p, \exp _{p}(v)\right)=|v|$ if $v \in b$ ([36], Proposition 6.10, p. 105). A subset $U$ is called convex if for each $p, q \in U$ there is a unique geodesic $x$ from $p$ to $q$ of length $\rho(p, q)$, contained entirely in $U$. Any point has a convex neighborhood ([36, 6-4, p.112).

Lemma 6.2. Let $M$ be a Riemannian manifold, $G$ a topological group acting isometrically and transitively on $M$ (the action $G \times M \rightarrow M$ is assumed continuous). Let $B\left(p_{0}, r\right)$ be a geodesic ball of $M$, contained in a convex set $W$. Assume there exists a compact $Y \subseteq G$ such that if $x, x^{\prime} \in B\left(p_{0}, r\right)$, then for some $g \in Y$ we have $g x=x^{\prime}$ and $\rho\left(x, g^{2} x\right)=2 \rho\left(x, x^{\prime}\right)$.

Let $U$ be any open set of diameter $<r$. Let $C$ be the collection of neighborhoods of $p_{0}$ of the form $\mathrm{cl}\left(g_{1} U \cap g_{2} U\right)$. Then $C$ strongly generates the topology at $p_{0}$.

Proof. It suffices to show that there are nonempty sets of the form $g_{1} U \cap g_{2} U$, of arbitrarily small diameter. For then by translation we may take these sets to contain $p_{0}$, and their closures will still have small diameter, and will strongly generate the topology at $p_{0}$.

Let $\bar{U}$ be the closure of $U$, and let $\delta<r$ be the diameter of $U$.

Find $p_{n}, q_{n} \in U$ with $\rho\left(p_{n}, q_{n}\right) \geq \delta-1 / n$, and find $g_{n} \in G$ with $g_{n} p_{n}=q_{n}$ and $\rho\left(p_{n}, g_{n}^{2} p_{n}\right)=2 \rho\left(p_{n}, q_{n}\right)$. By assumption, we may choose $g_{n}$ in a compact set, and all $p_{n}, q_{n}$ lie within a compact set (a closed ball of radius $r$ ). Refining the sequence $\left(p_{n}, q_{n}, g_{n}\right)$, we may thus assume it converges to a point $(p, q, g) \in \bar{U}^{2} \times G$, and we have $\rho(p, q)=\delta, \rho\left(p, g^{2} p\right)=2 \rho(p, q)=2 \delta$. It follows from uniqueness of the minimizing geodesic between $p$ and $g^{2} p$ that $\bar{B}(p, \delta) \cap \bar{B}\left(g^{2} p, \delta\right)=\{q\}$. By definition of $\delta$ we have $\bar{U} \subseteq \bar{B}(x, \delta)$ for any $x \in \bar{U}$. In particular, $\bar{U} \subseteq \bar{B}(p, \delta)$, and $\bar{U} \subseteq \bar{B}(q, \delta)$. From the latter we obtain $g \bar{U} \subseteq \bar{B}\left(g^{2} p, \delta\right)$. So $\bar{U} \cap g \bar{U}=\{q\}$.

The set $U \cap g_{n} U$ is nonempty, since $q_{n} \in U \cap g_{n} U$. It remains only to show that the diameter of $U \cap g_{n} U$ approaches 0 as $n \rightarrow \infty$.

Suppose otherwise; then there exist $\gamma>0$, and $a_{n}, b_{n} \in U \cap g_{n} U$ such that $\rho\left(a_{n}, b_{n}\right) \geq \gamma$ for infinitely many $n$. We can refine the sequence again to assume $a_{n} \rightarrow a, b_{n} \rightarrow b$; we have $\rho(a, b) \geq \gamma$, so $a \neq b$, and $a, b \in \bar{U} \cap g \bar{U}$. But we have seen that $\bar{U} \cap g \bar{U}$ consists of a single point, a contradiction.

The hypotheses of Lemma 6.2 are satisfied when $G$ is a Lie group, acting on itself by left translation, $M$ is $G$ with a left-invariant Riemannian metric, and $B\left(p_{0}, 2 r\right)$ is a geodesic ball. For then $Y=\bar{B}\left(p_{0}, r\right) \bar{B}\left(p_{0}, r\right)^{-1}$ is compact. For $x, x^{\prime} \in B\left(p_{0}, r\right)$, let $g=x^{\prime} x^{-1}, h=x^{-1} g x=x^{-1} x^{\prime}$, and let $|u|=\rho(1, u)$. Then $g x=x^{\prime}$. We have 
$\rho\left(x, x^{\prime}\right)=\rho\left(x^{\prime}, g^{2} x\right)=\rho\left(1, x^{-1} g x\right)=|h|, \rho\left(x, g^{2} x\right)=\rho\left(1, x^{-1} g^{2} x\right)=\left|h^{2}\right|$, so we have to show that $\left|h^{2}\right|=2|h|$. We have $h=\exp (v)$ for some $v$, where exp is the the exponential map at $1, h^{2}=\exp (2 v)$, and $\left|h^{2}\right|=|2 v|=2|v|=2|h|$.

Corollary 6.3 (Stabilizer theorem). Let $X$ be a near-subgoup of $G$.

- There exist a $\bigvee$-definable $\breve{G}$ and an $\bigwedge$-definable normal subgroup $\Gamma \subseteq \breve{G}$, both defined without parameters, such that $\breve{G} / \Gamma$ is bounded, and any definable $D$ with $\Gamma \leq D \leq \breve{G}$ is commensurable to $X^{-1} X$.

- There exist a connected Lie group $L$ and a homomorphism $\pi: \breve{G} \rightarrow L$ with dense image, and kernel $\Gamma$. If $D$ is a definable subset of $G$, write $\pi D$ for the closure of $\pi(D) . \pi$ intertwines the definable sets containing $\Gamma$, contained in $\breve{G}$ with the compact neighborhoods of $L$.

- There exist a uniformly definable family of definable sets $D_{a}$, and a definable set $E$, with $\partial \pi\left(D_{a}\right) \cap \pi E \subset \operatorname{int}(\pi(E))$ such that the neighborhoods of 1 of the form $\pi E \backslash \pi D_{a}$ generate the topology of $L$ at 1 .

Proof. The first two parts follow from Theorem 4.2 .

There remains to prove the uniform generation of the topology of $L$. Fix a leftinvariant Riemannian metric on $L$, and view $M=L$ as a Riemannian manifold. Let $p_{0}=1$ and let $r$ be as in Lemma 6.2 renormalizing, we may assume $r=4$. Write $B_{s}$ for $B(1, s)$. By the above remark there exists a definable $E$ with $\bar{B}_{5} \subseteq$ $\pi E \subseteq B_{6}$. Similarly there exists a definable $D$ such that $\pi D$ contains $\bar{B}_{9} \backslash B_{2}$ (so $\partial(\pi(D)) \cap E \subset \operatorname{int}(E))$ and is disjoint from $\bar{B}_{1}$. Then $U=\pi(E) \backslash \pi(D)=B_{2} \backslash \pi(D)$ is an open neighborhood of 1 . By Lemma 6.2, there exists $g, g^{\prime} \in B_{3}$ with $g U \cap g^{\prime} U$ of arbitrarily small diameter, and containing 1 . We compute $U \cap g U=(\pi(E) \cap$ $\pi(g E)) \backslash \pi(D \cup g D)=B_{2} \backslash \pi(D \cup g D)=\pi(E) \backslash \pi(D \cup g D)$, and similarly for $g U \cap g^{\prime} U$. The uniformly definable family is the family of unions $D \cup g D$.

6.4. The locally compact Lascar group. Let $T$ be a theory, $\mathbb{U}$ a universal domain, $\widetilde{E}$ a $\bigvee$-definable equivalence relation, $\Sigma$ an $\bigwedge$-definable equivalence relation, such that $\Sigma$ implies $\widetilde{E}$. Let $P$ be a complete type. Let $\tilde{a}$ be a class of $\widetilde{E}$ restricted to $P$, such that $\tau=\tilde{a} / \Sigma$ is bounded. Let $\pi: \tilde{a} \rightarrow \tilde{a} / \Sigma$ be the quotient map. Then $\tilde{a} / \Sigma$ admits a natural locally compact topology, generated by the complements of the images $\pi(D)$ of definable sets. $G=\operatorname{Aut}(\mathbb{U} / \tilde{a})$ acts on $\tau$. Let $K$ be the kernel of this action, and $L=G / K$. Then $L$ admits a natural locally compact group structure; we call it the locally compact Lascar group of $(\tilde{a}, \Sigma)$.

We have transposed from definable groups (as in Theorem 6.3) to automorphism groups. In both cases, the set of conjugates of a definable set lie in a uniformly definable family. We will use this in Lemma 6.6 below.

6.5. The compact Lascar group. So far, the case where $\tilde{a}$ is a definable set and $L$ is compact has been useful. For simplicity, we will also restrict to this case in the statement below. For the rest of this section we assume $\widetilde{E}$ is the indiscrete equivalence relation, so $\tilde{a}=P$ and $\tau=P / \Sigma$ is compact. We do not expect any trouble in generalizing to the locally compact case.

Lemma 6.6. Let $L^{\prime}=L / N$ be a finite-dimensional quotient of $L$, so $N$ is a compact normal subgroup and $L^{\prime}$ is a compact Lie group. For large enough $k$, $L^{\prime}$ has a regular orbit on $\tau^{k} / N=P^{k} / N$. Let $\tau^{\prime}$ be such an orbit. There exists a uniformly definable family of definable sets $D_{a}$, such that the sets $\tau^{\prime} \backslash \pi\left(D_{a}\right)$ strongly generate the topology on $\tau^{\prime}$ at every point. 
Proof. For $x \in \tau$, let $S_{x}$ be the stabilizer of $x$. Let $\Xi$ be the set of finite subsets of $\tau$. For $u \in \Xi$, let $S_{u}=\bigcap_{x \in u} S_{x}$. We have $\bigcap_{x \in \tau} S_{x}=K$. Since $N$ is compact, $\bigcap_{u \in \Xi} S_{u} N=K N=N$. (Let $a \in \bigcap_{u \in \Xi} S_{u} N$. Pick an ultrafilter on $\Xi$ including all sets of the form $\{u: x \in u\}$. Write $a=s_{u} n_{u}$ with $n_{u} \in N, s_{u} \in S_{u}$. Then $s_{u} \rightarrow s$ and $n_{u} \rightarrow n$ for some $s, n$. We have $s \in \bigcap_{x} S_{x}=K$ and $n \in N$.)

Now $L^{\prime}$ is a compact Lie group, so it has no infinite descending sequences of closed subgroups. Thus for some finite tuple $u=\left(x_{1}, \ldots, x_{k}\right)$ we have $S_{u} N=N$. It follows that $\tau^{\prime}=L^{\prime} x$ is a regular orbit in $\tau^{k} / N$. The uniformity statement follows from Lemma 6.2, as in the proof of Theorem 6.3, since compactness is assumed, we can take $E$ to be the entire ambient sort.

An earlier version of this section attempted an application to SOP theories, but in this, Krzysztof Krupinski found a gap.

\section{Groups With Large approximate subgroups}

In this section we aim to prove:

Theorem 7.1. Let $G_{0}$ be a finitely generated group, $k \in \mathbb{N}$. Assume $G_{0}$ has a cofinal family of $k$-approximate subgroups (i.e., any finite $F_{0} \subset G_{0}$ is contained in one). Then $G_{0}$ is nilpotent-by-finite.

This generalizes Gromov's theorem [18, asserting the same conclusion if $G_{0}$ has polynomial growth. There is by now a small family of proofs of Gromov's theorem and extensions, descending from either Gromov's original proof or Kleiner's; the first may have been [13, and the most recent, [46]. I believe all view the group as a metric space, via the Cayley graph, and analyze it either geometrically or analytically.

We will consider an arbitrary sequence of approximate subgroups, rather than balls in the Cayley graph. A Lie group $L$ lies at the heart of the proof, as in the case of Gromov's. While Gromov's group arises in the automorphism group of the Cayley graph "viewed from afar", we find $L$ and a homomorphism $h: G_{0} \rightarrow L$ using the model-theoretic/measure-theoretic construction Theorem 3.5 which has no metric aspect.

Beyond this point, our proof will adhere very closely to the outline of Gromov's. If the homomorphism into $L$ is trivial, we conjugate it to a nontrivial one in exactly the way taken by Gromov, succeeding unless $G_{0}$ is already virtually Abeliar 3 (in which case we are already done). We now use the earlier Corollary 5.12 covering the linear case to show that the image is essentially solvable, and hence a nontrivial homomorphism into an Abelian group can be obtained. Gromov used the Tits alternative at the parallel point. We show that the kernel satisfies the same assumptions as $G_{0}$; here we make some further use of Lie theory. Induction is carried out on the Lie dimension, rather than the growth rate exponent which is not available to us; we conclude that the group is polycyclic-by-finite, and in particular virtually solvable. To pass from the polycyclic solvable to the nilpotent case, we quote Tao [53] or Breuillard-Green [4] where Gromov cited Milnor-Wolf.

We will see along the way that $G_{0}$ is polycyclic-by-finite with $d$ infinite cyclic factors, where $d$ is the dimension of the associated Lie group.

\footnotetext{
${ }^{3}$ We say that a group $G_{0}$ is virtually $P$ if some finite index subgroup is $P$.
} 
An alternative statement is that when $G_{0}$ is not nilpotent-by-finite, then for some finite $F_{0} \subset G_{0}, G_{0}$ has no $k$-approximate subgroups containing $F_{0}$. If one wishes to seriously use the ambient group $G_{0}$, some hypothesis on containing sets of generators is necessary (e.g. since any countable family of finitely generated groups embeds jointly in a single one).

The strongest possible general conjecture on the structure of $k$-approximate subgroups would be this: for some $k^{\prime}, k^{\prime \prime}$, any $k$-approximate subgroup of a group $G$ is $k^{\prime}$-commensurable with one induced by a map into a $k^{\prime \prime}$-nilpotent group. Here we say that $X$ is induced by $h$ if $h$ is a homomorphism on some subgroup $A$ of $G$ into a group $N$, and $X=h^{-1} h(X)$. Statements in this vein, possibly restricted to approximate subgroups of a fixed group, have been suggested by Helffgott, E. Lindenstrauss, Breuillard and Tao.

The conjugation method used in the present section would be powerless in the following scenario: $X_{n}$ is a $k$-approximate subgroup of the alternating group $A_{n}$, and $X_{n}$ is conjugation-invariant.

Towards the proof of Theorem 7.1, we will study the following situation $\diamond$ :

- A language with two sorts $G, \Phi ; G$ carries a group structure; a relation on $G \times \Phi$ defines a family of definable subsets of $G,\left(X_{c}: c \in \Phi\right)$. Additional structure is allowed.

- $M^{*}$ is a saturated structure, with an elementary submodel $M$.

- $G_{0}=G(M)$ is finitely generated.

- $X=X_{c^{*}}$ is a $c^{*}$-definable subset $X$ with $G(M) \subset X\left(c^{*}\right.$ is an element of $\left.\Phi\left(M^{*}\right)\right)$.

- For all $c \in \Phi(M), X_{c}$ is finite.

- There is an $\bigwedge$-definable subgroup $\Gamma$ of $G$, and a $\bigvee$-definable subgroup $\widetilde{G}$, with $\Gamma \subseteq X \subseteq \widetilde{G}$, and $\widetilde{G} / \Gamma$ bounded. $\Gamma, \widetilde{G}$ are defined over some small subset of $M^{*}$.

- Any subgroup of $G(M)$ has the form $S(M)$ for some 0-definable subgroup $S$ of $G$.

In this situation, note:

(1) We may replace $\widetilde{G}$ by the group generated by $X$, without disturbing the hypotheses.

(2) Let $G^{\prime}$ be a 0-definable subgroup of $G ; X_{c}^{\prime}=X_{c} \cap G^{\prime} ; \Gamma^{\prime}=\Gamma \cap G^{\prime}, \widetilde{G}^{\prime}=$ $\widetilde{G} \cap G^{\prime}$. Then $(\diamond)$ holds with the new data, except possibly for the finite generation of $G^{\prime}(M)$. When $G^{\prime}$ has finite index in $G$, this too holds.

(3) There exists a $\bigvee$-definable $\breve{G} \leq \widetilde{G}$ and a normal $\bigwedge$-definable subgroup $\Gamma^{\prime}$ of $\breve{G}$ containing $\breve{G} \cap \Gamma$, such that $\breve{G} / \Gamma^{\prime}$ is a connected Lie group. (This is Theorem 4.2. We have $\breve{G} \cap \Gamma \subseteq \Gamma^{\prime}$ since the image of $\breve{G} \cap \Gamma$ in $\breve{G} / \Gamma^{\prime}$ is a compact normal subgroup, hence trivial.)

(4) $X$ is contained in finitely many cosets of $\breve{G}$ (the image of $X$ modulo $\breve{G}$ is a compact subset of the discrete space $\widetilde{G} / \breve{G})$.

(5) $G_{0} \cap \breve{G}$ has finite index in $G_{0}$ (since $G_{0} \subseteq X$, by (4), $G_{0}$ is contained in finitely many cosets of $\breve{G}$, equivalently of $G_{0} \cap \breve{G}$ ).

(6) Let $H_{0}=G_{0} \cap \breve{G}$; let $H$ be a 0-definable group, with $H_{0}=H(M)$. So $H$ has finite index in $G$. Let $\widetilde{H}=\widetilde{G} \cap H$, let $Y$ be a definable subset of $\widetilde{H}$ commensurable with $X$, and containing $X \cap \widetilde{H}$, with corresponding family $\left(Y_{c}: c \in \Phi^{\prime}\right)$. We choose $\Phi^{\prime} \subset \Phi$ so that $Y_{c}$ is commensurable to $X_{c}$ for 
$c \in \Phi^{\prime}$ : in particular, $Y_{c}$ is finite for $c \in \Phi^{\prime}(M)$. So $H(M) \subset Y$. Now the hypotheses $\diamond$ hold for $(H, Y, \widetilde{H}, \Gamma \cap H)$. Let $\breve{H}=\breve{G} \cap H$ and $\Gamma^{\prime \prime}=\Gamma^{\prime} \cap H$. Then $\breve{H} / \Gamma^{\prime \prime}$ has finite index in $\breve{G} / \Gamma^{\prime}$, but the latter is connected, so they are equal. Hence $\breve{H} / \Gamma^{\prime \prime}$ is connected, and $H_{0}=G_{0} \cap \breve{G} \subseteq \breve{G} \cap H=\breve{H}$. Now we are in the same situation $\diamond$, but have in addition $H_{0} \leq \breve{H}$.

Before entering the proof proper, we can clarify the meaning of this setup by looking at the Lie rank zero case.

Lemma 7.2. Assume $\diamond$, and further assume that $\widetilde{G} / \Gamma$ is totally disconnected. Then $G=G_{0}$ is finite.

Proof. Being totally disconnected, $\widetilde{G} / \Gamma$ contains a compact open subgroup $C$. If $\psi: \breve{G} \rightarrow L$ is the canonical map, then $H=\psi^{-1}(C)$ is a definable group by compactness of $C$ and is commensurable with $X$ by openness. Since $X$ contains $G_{0}=G(M), H$ is covered by finitely many cosets of $G_{0}$, so $H \cap G_{0}$ has finite index in $G_{0}$. In particular it is finitely generated. Let $F_{1}$ be a finite set of generators for $H \cap G_{0}$. Since $M \prec M^{*}$, there exists a definable group $H_{c}$ containing $F_{1}$ and commensurable with $X_{c}$, for some $c \in \Phi(M)$; so $H_{c}$ is finite. It follows that the group generated by $F_{1}$ is finite, i.e. $H \cap G_{0}$ is finite; thus $G_{0}$ is finite.

We will need some lemmas on finite generation. First, if $E$ is a finitely generated group, $N$ a normal subgroup with $E / N$ finitely presented, then $N$ is finitely generated as a normal subgroup. (In particular when $E / N$ is finite, this implies the finite generation of $N$, a well-known statement used above.) This in fact is valid for any equational class: If $E$ is finitely generated and $N$ is a congruence with $E / N$ finitely presented, then $N$ is finitely generated as a congruence. Indeed let $F$ be a finitely generated free algebra in this equational class, and $h: F \rightarrow E$ a surjective homomorphism. Let $g: F \rightarrow E / N$ be the composition $F \rightarrow E \rightarrow E / N$. Since $E / N$ is finitely presented, $g$ has a finitely generated kernel $K$. Thus $N=h(K)$ is finitely generated.

A $\bigvee$-definable subgroup is called definably generated if it is generated by a definable subset. If $G$ is a topological group, let $G^{0}$ denote the connected component of 1 ; it is a closed normal subgroup of $G$.

Let $H$ be a sufficiently saturated group (with possible additional structure), $\breve{H}$ be a $\bigvee$-definable subgroup, $\Gamma$ an $\bigwedge$-definable subgroup, with $\Gamma \unlhd \breve{H}$. Let $\pi: \breve{H} \rightarrow \breve{H} / \Gamma$ be the quotient map. Recall the logic topology on $\breve{H} / \Gamma$ from $\S 4$. In particular, a subset $Z$ of the quotient is compact iff $\pi^{-1}(Z)$ is contained in a definable set.

Lemma 7.3. Let $H, \breve{H}, \Gamma, \pi$ be as above, and assume $A=\breve{H} / \Gamma$ is locally compact. Assume $A / A^{0}$ is finitely generated. Then $\breve{H}$ is definably generated.

Proof. Let $U$ be a compact neighborhood of 1 in $A$. Then $\pi^{-1}(U)$ is contained in a definable subset $D$ of $\breve{H}$. $U$ generates an open subgroup of $A$; this open subgroup is also closed, and must contain $A^{0}$. On the other hand, $A / A^{0}$ is generated by finitely many elements $\pi\left(h_{1}\right), \ldots, \pi\left(h_{r}\right)$. Let $D^{\prime}=D \cup\left\{h_{1}, \ldots, h_{r}\right\}$. Since $D$ contains $\operatorname{ker} \pi$ and $\pi \breve{H}$ is generated by $\pi\left(D^{\prime}\right)$, it follows that $\breve{H}$ is generated by $D^{\prime}$.

In fact if $A / A^{0}$ is $m$-generated, the proof shows that $\breve{H}$ is generated by $Y$ along with $m$ additional elements, whenever $Y$ is a definable set containing $\Gamma$. 
Lemma 7.4. Let $G$ be a sufficiently saturated group (with additional structure), $H$ a definable normal subgroup with $G / H$ Abelian. Let $\breve{G}$ be $a \bigvee$-definable subgroup of $G, \Gamma$ an $\bigwedge$-definable subgroup, with $\Gamma \unlhd \breve{G}$ and $E=\breve{G} / \Gamma$ a connected Lie group. Then $H \cap \breve{G}$ is definably generated.

Proof. Let $\breve{H}=H \cap \breve{G}$, and $\pi: \breve{G} \rightarrow E$ be the canonical map. By Remark 4.10, $\pi \mid \breve{H}$ induces an isomorphism of topological groups $\breve{H} /(\breve{H} \cap \Gamma) \cong \pi(\breve{H})$.

Since $H$ is normal in $G, \breve{H}$ is normal in $\breve{G}$, so $\pi(\breve{H})$ is normal in $E$, and hence so is $\pi(\breve{H})^{0}$. The commutator subgroup $[E, E]$ is contained in $\pi(\breve{H})^{0}$, so the quotient $E / \pi(\breve{H})^{0}$ is isomorphic to $\mathbb{R}^{n} \times \mathbb{R}^{m} / \mathbb{Z}^{m}$. The image of $\pi(\breve{H})$ in $E / \pi(\breve{H})^{0}$ contains no nontrivial connected groups, so it is discrete. Now it is well known that a discrete subgroup of $\mathbb{R}^{n} \times \mathbb{R}^{m} / \mathbb{Z}^{m}$ is finitely generated, indeed admits a generating set with at most $n+m$ elements. By Lemma 7.3, $H \cap \breve{G}$ is definably generated.

The next lemma will play an essential role in the proof, allowing the key Lemma 7.6 to be propagated. It continues to hold if $G / N$ is assumed to be nilpotent, rather than Abelian; indeed it suffices to find a sequence of definable subgroups $G=H_{1} \supset \cdots \supset H_{k}=H$ with $H_{i+1}$ normal in $H_{i}$, and $H_{i} / H_{i+1}$ Abelian, and apply the lemma inductively.

Lemma 7.5. Assume $\diamond$ holds, and let $N$ be a 0 -definable normal subgroup of $G$. Then $\diamond$ holds if $G$ is replaced by $G / N$, and $X, \widetilde{G}, \Gamma$ by their images in $G / N$.

If $G / N$ is finite or Abelian, then $\diamond$ holds if $G, X, \widetilde{G}, \Gamma$ are replaced by $N, X \cap$ $N, \widetilde{G} \cap N, \Gamma \cap N$.

Proof. The first statement is straightforward; so is the second, except for the finite generation of $N_{0}=N(M)$. We proceed to show this. In case $G / N$ is finite, so is $G_{0} / N_{0}$ since $M$ is an elementary submodel, so finite generation is clear. Assume therefore that $G / N$ is Abelian.

Let $G_{1}=G_{0} \cap \breve{G}$. As $G_{1}$ has finite index in $G_{0}$ by (5), it is a finitely generated group.

Let $N_{1}=N \cap G_{1}$. Since $G_{1} /\left(N \cap G_{1}\right)$ is finite or Abelian, it is a finitely presented group. By the remarks above, $N_{1}$ is finitely generated as a normal subgroup of $G_{1}$.

Let $g_{1}, \ldots, g_{r}$ be generators for $G_{1}$, and let $T_{i}(x)=g_{i}^{-1} x g_{i}$. Then $N_{1}$ is finitely generated as a group with these operators. Let $Y$ be a finite subset of $N_{1}$ such that $N_{1}$ is generated by $Y$ under multiplication and the operators $T_{i}$.

By Lemma 7.4, $\breve{G} \cap N$ is generated by an $M^{*}$ - definable set $U$. We may take $Y \subset U=U^{-1}$. Since $\breve{G}$ and $N$ are closed under the operators $T_{i}$ (as $g_{i} \in \breve{G}$ and $N$ is normal), we have $T_{i}(U) \subset U \cdots U=U^{m}$ for some $m$. Since $U$ is a definable subset of $\breve{G}$, it is contained in finitely many translates of $X$. Now $M$ is an elementary submodel of $M^{*}$. So there exists an $M$-definable set $U^{\prime} \subset N$ containing $Y$, with $T_{i}\left(U^{\prime}\right) \subset U^{\prime} \cdots U^{\prime}$, and $U^{\prime}$ contained in finitely many translates of some $X_{c}, c \in \Phi\left(M_{0}\right)$. From the last property it follows that $U^{\prime}$ is finite; so $U^{\prime} \subset M$ and hence $U^{\prime} \subset G_{0}$. Thus $U^{\prime} \subset N_{1}$. Moreover the group generated by $U^{\prime}$ is closed under the operators $T_{i}$, and contains $Y$. So it equals $N_{1}$. This shows that $N_{1}$ is a finitely generated group. Since it has finite index in $N_{0}=N \cap G_{0}$, it follows that $N_{0}$ too is a finitely generated group.

Lemma 7.6. Assume $\diamond$ holds, and $G_{0}$ is infinite. Then there exists a normal subgroup $N_{0}$ of $G_{0}$ with $G_{0} / N_{0}$ virtually Abelian, and infinite. 
Proof. Let $G_{0}^{\prime}=G_{0} \cap \breve{G}$. By note (5) above, $G_{0}^{\prime}$ has finite index in $G_{0}$. If $N_{0}^{\prime}$ is normal in $G_{0}^{\prime}$ with infinite virtually Abelian quotient, let $N_{0}$ be the intersection of the finitely many $G_{0}$-conjugates of $N_{0}^{\prime}$; then $G_{0} / N_{0}$ is infinite and virtually Abelian. Thus proving the lemma for $G_{0}^{\prime}$ would imply it for $G_{0}$. By note $(6)$, the hypotheses hold for $G_{0}^{\prime}$; so we may assume $G_{0} \leq \breve{G}$.

Let $L=\breve{G} / \Gamma^{\prime}$ as in note $(3), d=\operatorname{dim}(L)$, and consider the natural homomorphism $\psi: \breve{G} \rightarrow L$. Note that $\psi\left(G_{0}\right)$ has a cofinal system of $k$-approximate subgroups. By Corollary 5.12 any finite subset $w$ of $\psi\left(G_{0}\right)$ is contained in at most $k^{\prime \prime}$ cosets of a $(d+2)$-solvable subgroup $S_{w}$ of $L$. Taking an ultraproduct, $L$ embeds in an ultraproduct of itself, in such a way that the image of $\psi\left(G_{0}\right)$ is contained in at most $k^{\prime \prime}$ cosets of a $(d+2)$-solvable group $S$. Thus $\psi\left(G_{0}\right)$ has a solvable subgroup $S^{\prime}$ of finite index. If $S^{\prime}$ is infinite, then it contains a subgroup $S^{\prime \prime}$ of finite index, such that $S^{\prime \prime} /\left[S^{\prime \prime}, S^{\prime \prime}\right]$ is infinite. Thus $\psi^{-1}\left(S^{\prime \prime}\right)$ is a finite index subgroup of $G_{0}$, and $\psi^{-1}\left(\left[S^{\prime \prime}, S^{\prime \prime}\right]\right)$ is a normal subgroup with infinite Abelian quotient. So we are done unless $S^{\prime}$ above is finite, i.e. $\psi\left(G_{0}\right)$ is finite, so that a finite index subgroup $H_{0}$ of $G_{0}$ is contained in $\Gamma^{\prime}$. We have $H_{0}=H(M)$ for some 0-definable subgroup $H$ of $G$.

For $g \in G$, let $a d_{g}(x)=g^{-1} x g$, and let $\tau_{g}=a d_{g} \mid H_{0}$. Let $J=\left\{g \in G: \tau_{g}\left(H_{0}\right) \leq\right.$ $\breve{G}$. If $g \in J$, we may repeat the previous paragraph with $\psi \circ \tau_{g}$ in place of $\psi$. Thus again we are done unless $\psi \circ \tau_{g}\left(H_{0}\right)$ is finite for any $g \in J$. We thus assume this is the state of affairs.

The rest of the proof is a straightforward transcription of the corresponding part of [18. By Jordan's theorem [29], since $\psi \circ \tau_{g}\left(H_{0}\right)$ is a finite subgroup of the Lie group $L$, it has an Abelian subgroup $S_{g}$ of index $\leq \mu$, with $\mu$ independent of $g$. If $\psi \circ \tau_{g}$ can have arbitrarily large finite size for $g \in J$, taking an ultraproduct, we obtain a homomorphism to a group with an infinite Abelian subgroup of index $\leq \mu$. Thus in this case too the lemma is proved, and we may assume that $\psi \circ \tau_{g}\left(H_{0}\right)$ has size $\leq \mu^{\prime}$ for some fixed $\mu^{\prime}$.

Let $F_{1}$ be a finite set of generators for $H_{0}$. Let $U$ be a neighborhood of the identity in the Lie group $L$, such that if $u \in U$ is an element of order $\leq \mu^{\prime}$, then $u=1$. (For instance we can take a neighborhood $V$ of the Lie algebra on which the exponential map is injective, and then let $U=\exp \left(\left(1 / \mu^{\prime}\right) V\right)$.) Since $\{1\}$ is closed and $U$ is open, there exists a definable set $D_{2} \subset G$ with $\Gamma^{\prime} \subset D_{2} \subset \psi^{-1}(U)$. Since $F_{1}^{-1} \Gamma^{\prime} F_{1}=\Gamma^{\prime} \subset D_{2}$, we can find a definable set $D$ with $\Gamma^{\prime} \subset D$ and $F_{1}^{-1} D F_{1} \subset D_{2}$. Any subgroup of $D_{2}$ of size $\leq \mu^{\prime}$ is trivial. Now if $\tau_{g}\left(F_{1}\right) \subset D_{2}$, then $\tau_{g}\left(H_{0}\right) \leq \breve{G}$, so $g \in J$. Hence $\psi \circ \tau_{g}\left(H_{0}\right)$ has size $\leq \mu^{\prime}$, but $\psi \circ \tau_{g}\left(F_{1}\right)$ is a set of elements of $U$, and any such nonidentity element has order $>\mu^{\prime}$; so $\psi \circ \tau_{g}\left(F_{1}\right)$ must reduce to the identity element of $L$. Hence if $\tau_{g}\left(F_{1}\right) \subset D_{2}$, then $\tau_{g}\left(H_{0}\right) \subset \Gamma^{\prime}$, and in particular $\tau_{g}\left(F_{1}\right) \subset D$.

Let $W=\left\{g: \tau_{g}\left(F_{1}\right) \subset D\right\}=\left\{g: \tau_{g}\left(F_{1}\right) \subset D_{2}\right\}$. If $g \in W$ and $f \in F_{1}$, then $g f \in W$, since $(g f)^{-1} F_{1} g f \subseteq f^{-1} D f \subseteq D_{2}$. So $W$ is a definable, right $F_{1}$-invariant set. Now in the model $M$, any definable, right $F_{1}$-invariant set is empty or contains $H_{0}$. Since $M \prec M^{*}$, it follows that $W=\emptyset$ or $W$ contains $H$. We have $1 \in W$, as $H_{0} \leq \Gamma^{\prime} \leq D$. So all $H$-conjugates of $F_{1}$ are contained in $D$. Note that $D$ is contained in the union of finitely many translates of $X$. It follows that all $H_{0^{-}}$ conjugates of $F_{1}$ are contained in finitely many translates of some $X_{c}, c \in \Phi(M)$. In this case each element of $F_{1}$ has centralizer of finite index in $H_{0}$, so $H_{0}$ has a center of finite index; we may take $G_{0}^{\prime}$ to be this center, and $N=1$. 
We will need some elementary group-theoretic discussion before proceeding. We define a group $H$ to be 0 -polycyclic if it is trivial, and to be $(d+1)$-polycyclic if it has a $d$-polycyclic normal subgroup $N$, with $H / N$ a finitely generated Abelian group of rank 1. In particular, $H$ is $d$-solvable.

For $d \geq 0$, we say that a finitely generated group $H$ is almost $d$-polycyclic if it has subgroups $H_{2 d+2} \unlhd H_{2 d+1} \unlhd H_{2 d} \unlhd \cdots \unlhd H_{1}=H$ with $H_{2 i} / H_{2 i+1}$ finite $(0 \leq i \leq d)$, and $H_{2 i+1} / H_{2 i+2} \cong \mathbb{Z}(0 \leq i \leq d)$, and $H_{2 d+2}=0$. By reverse induction on $i$ we see that each $H_{i}$ is finitely generated in this situation.

These definitions differ in that the quotients $H_{2 i} / H_{2 i+1}$ are not required to be Abelian, but if $H$ is almost $d$-polycyclic, then it does have a $d$-polycyclic normal subgroup of finite index. To show this we may pass to a finite index subgroup, so we may assume $H$ has an almost $(d-1)$-polycyclic normal subgroup $N$ with $H / N \cong \mathbb{Z}$. Like all almost polycyclic groups, $N$ is finitely generated. Using the induction hypothesis, let $N_{1}$ be a $(d-1)$-polycyclic subgroup of $N$, with $\left[N: N_{1}\right]=r<\infty$. As $N$ is finitely generated, it has only finitely many subgroups of index $r$. Let $N_{2}$ be their intersection. Then $N_{2}$ is $(d-1)$-polycyclic and is characteristic in $N$, hence normal in $H . H / N_{2}$ contains the finite group $N / N_{2}$ as a normal subgroup; within $H / N_{2}$, the centralizer of $N / N_{2}$ has the form $H_{1} / N_{2}$, with $H_{1}$ a finite index subgroup of $H$. Now $H_{1} /\left(N \cap H_{1}\right) \cong \mathbb{Z}$, while $\left(N \cap H_{1}\right) / N_{2}$ is a finite central subgroup of $H_{1} / N_{2}$; so $H_{1} / N_{2}$ is a finitely generated Abelian group of rank 1 . Thus $H_{1}$ is $d$-polycyclic.

Lemma 7.7. Assume $\diamond$. Then $G_{0}=G(M)$ is polycyclic-by-finite (and in particular solvable-by-finite).

Proof. We use induction on $d=\operatorname{dim}(L), L=\breve{G} / \Gamma^{\prime}$. If $d=0$, then $\widetilde{G} / \Gamma$ is totally disconnected; hence $G_{0}$ is finite by Lemma 7.2. For higher $d$, we use Lemma 7.6. By note $(2)$ to $\diamond$ we may pass to a finite index subgroup; so we may assume there exists a 0-definable normal subgroup $N$ of $G$, with $G / N$ infinite Abelian. By Lemma 7.5, the hypotheses $\diamond$ hold for $N, X \cap N, \widetilde{G} \cap N, \Gamma \cap N$, and also for the images in $G / N$. By Lemma 7.2 applied to $G / N$, we see that the image of $\widetilde{G}$ in $G / N$ has Lie rank $\geq 1$. By Remark 4.10 (3) it follows that $\widetilde{G} \cap N$ has Lie rank $<d$. So the inductive hypothesis applies, and $N_{0}=N(M)$ is polycyclic-by-finite. Thus $G_{0}$ is almost polycyclic and hence also polycyclic by finite.

We are now essentially in the solvable case, and can quote either [53], or [4]. Polycyclicity is a strong additional tool, and with it one may be able to reduce to the sum-product phenomenon for fields somewhat more rapidly; a model-theorist is reminded here of Zilber's arguments in the 1970s, connecting solvable groups of finite Morley rank with definable fields. We will simply invoke 4]; thanks to Emmanuel Breuillard for pointing out a nicer path to this paper than we had initially.

Lemma 7.8. Assume $\diamond$. Then $G_{0}=G(M)$ is nilpotent-by-finite.

Proof. By Lemma 7.6, $G_{0}$ has a normal subgroup $N_{0}$ with $G_{0} / N_{0}$ virtually Abelian; as we just saw, $N_{0}$ satisfies $\diamond$, has lower Lie rank, and so inductively is nilpotent by finite. Passing to a finite index subgroup, we may assume $G_{0}$ is solvable, as well as polycyclic. Now any polycyclic group is linear over $\mathbb{C}$; see chapter 4 of 45] for a stronger result, due to Auslander-Swan. Hence $G_{0}$ can be viewed as a solvable subgroup of $G L_{n}(\mathbb{C})$; according to [4, every $k$-approximate subgroup of 
$G_{0}$ is covered by a bounded number of cosets of an ( $\left.n-1\right)$-step nilpotent subgroup of $G L_{n}(C)$. Taking the ultraproduct of the cofinal family of approximate groups associated with $G_{0}$, we see that $X$ and hence $G_{0}$ are covered by finitely many cosets of an $(n-1)$-step nilpotent group; hence $G_{0}$ is itself virtually nilpotent.

Proof of Theorem 17.1. Let $\left(X_{c}: c \in \Phi_{0}\right)$ be the given family of $k$-approximate subgroups of $G_{0}$. Consider the two-sorted structure $\left(G, \Phi_{0}, \cdot, E\right)$ where $(x, c) \in E$ if $x \in X_{c}$. Enrich it by adding a predicate for each subgroup of $G_{0}$. Further enrich the language by closing under probability quantifiers as in 22.6 . Let $M$ be the resulting structure, and let $M^{*}$ be a saturated elementary extension. By saturation and by the cofinality of the $X_{c}$, there exists $c^{*} \in \Phi\left(M^{*}\right)$ with $G_{0} \subset X_{c^{*}}$. All clauses of $\diamond$ are now clear, so by Lemma 7.8, $G_{0}$ is nilpotent-by-finite.

\section{ACKNOWLEDGMENT}

This paper was prepared for a talk at the Cherlin Bayramı in Istanbul in June 2009. The author is grateful to Dugald Macpherson for a valuable conversation on this subject in Leeds. Thanks also to Zoé Chatzidakis, Lou van den Dries, Ward Henson, Itay Kaplan, Krzysztof Krupinski, Elon Lindenstrauss, Dugald, Anand Pillay, Françoise Point, Laci Pyber, Tom Scanlon, Pierre Simon, Terry Tao, Alex Usvyatsov, and the two anonymous referees, for many useful comments.

\section{REFERENCES}

1. Ben Yaacov, Itaï; Usvyatsov, Alexander, Continuous first order logic and local stability. Trans. Amer. Math. Soc. 362 (2010), no. 10, 5213-5259. MR.2657678

2. Bergman, George M.; Lenstra, Hendrik W., Jr., Subgroups close to normal subgroups. J. Algebra 127 (1989), no. 1, 80-97. MR1029404 (91a:20004)

3. Breuillard, Emmanuel; Green, Ben; Terence, Tao, Approximate subgroups of linear groups, arXiv:1005.1881.

4. Breuillard, Emmanuel; Green, Ben, Approximate groups, II: the solvable linear case, arXiv:0907.0927.

5. Bourgain, J.; Katz, N.H.; Tao, T.C., A sum-product estimate in finite fields and applications, Geom. Funct. Anal. 14 (2004), no. 1, 27-57. MR2053599 (2005d:11028)

6. Chang, C. C.; Keisler, H. J., Model theory. Third edition. Studies in Logic and the Foundations of Mathematics, 73. North-Holland Publishing Co., Amsterdam, 1990. MR 1059055 (91c:03026)

7. Chang, Mei-Chu, Product theorems in $\mathrm{SL}_{2}$ and $\mathrm{SL}_{3}$. J. Inst. Math. Jussieu 7 (2008), no. 1, 1-25. MR 2398145 (2009d:20112)

8. Cherlin, Gregory; Hrushovski, Ehud, Finite structures with few types. Annals of Mathematics Studies, 152. Princeton University Press, Princeton, NJ, 2003, vi+193 pp. MR 1961194 (2004c:03037)

9. Curtis, Charles W.; Reiner, Irving, Representation theory of finite groups and associative algebras. Reprint of the 1962 original. AMS Chelsea Publishing, Providence, RI, 2006. xiv+689 pp. MR2215618 (2006m:16001)

10. Dinai, Oren, Expansion properties of finite simple groups. Ph.D. thesis. The Hebrew University of Jerusalem (2009). arXiv:1001.5069.

11. Elekes, György; Kirély, Zoltãn, On the combinatorics of projective mappings. (English summary) J. Algebraic Combin. 14 (2001), no. 3, 183-197. MR.1869409 (2003e:52034)

12. van den Dries, L., notes in http://www.math.uiuc.edu/ vddries/.

13. van den Dries, L.; Wilkie, A. J., Gromov's theorem on groups of polynomial growth and elementary logic. J. Algebra 89 (1984), no. 2, 349-374. MR751150 (85k:20101)

14. Erdös, P.; Szemerédi, E., On sums and products of integers. Studies in pure mathematics, 213 - 218, Birkhäuser, Basel, 1983. MR820223(86m:11011)

15. Gleason, A. M., The structure of locally compact groups. Duke Math. J. 18 (1951), 85-104. MR0039730 (12:589a) 
16. Goldbring, Isaac, Hilbert's fifth problem for local groups. Ann. of Math. (2) 172 (2010), no. 2, 1269-1314. MR2680491 (2011f:22010)

17. Green, Ben; Sanders, Tom, A quantitative version of the idempotent theorem in harmonic analysis, Annals of Mathematics, 168 (2008), 1025-1054. MR2456890(2010c:11013)

18. Gromov, Mikhael, Groups of polynomial growth and expanding maps. Inst. Hautes Études Sci. Publ. Math. No. 53 (1981), 53-73. MR623534 (83b:53041)

19. Halmos, P, "Measure Theory," Van Nostrand, Princeton, NJ, 1950. MR0033869 (11:504d)

20. Helfgott, H. A., Growth and generation in $\mathrm{SL}_{2}(\mathbb{Z} / p \mathbb{Z})$. Ann. of Math. (2) 167 (2008), no. 2, 601-623. MR2415382 (2009i:20094)

21. Helfgott, H. A., Growth in $S L_{3}(Z / p Z)$, arXiv:0807.2027.

22. Hewitt, E.; Savage, L.J., Symmetric measures on Cartesian products. Trans. Amer. Math. Soc., 80 (1955), 470-501. MR0076206 (17:863g)

23. Hrushovski, Ehud, Pseudo-finite fields and related structures. Model theory and applications, 151-212, Quad. Mat., 11, Aracne, Rome, 2002. MR2159717 (2006d:03059)

24. Hrushovski, Ehud; Wagner, Frank, Counting and dimensions. Model theory with applications to algebra and analysis. Vol. 2, 161-176, London Math. Soc. Lecture Note Ser., 350, Cambridge Univ. Press, Cambridge, 2008. MR2436141 (2009k:03042)

25. Hrushovski, Ehud; Peterzil, Ya'acov; Pillay, Anand, Groups, measures, and the NIP. J. Amer. Math. Soc. 21 (2008), no. 2, 563-596 (section 7). MR2373360 (2008k:03078)

26. Hrushovski, Ehud; Pillay, Anand, On NIP and invariant measures, arXiv:0710.2330.

27. Hrushovski, Ehud; Pillay, Anand; Simon, Pierre, Generically stable and smooth measures in NIP theories, arXiv:1002.4763.

28. Kallenberg, Olav, On the representation theorem for exchangeable arrays. J. Multivariate Anal. 30 (1989), no. 1, 137-154. MR.1003713 (90j:60034)

29. Jordan, Camille, Mémoire sur les équations différentielles linéaires intégrale algébriques, Crelle 84 (1878) pp. 89-215.

30. Kaplansky, Irving, Lie algebras and locally compact groups. Reprint of the 1974 edition. Chicago Lectures in Mathematics. University of Chicago Press, Chicago, IL, 1995. xii+148 pp. MR 1324106 (96a:22008)

31. Kim, Byunghan; Pillay, Anand, Simple theories. Joint AILA-KGS Model Theory Meeting (Florence, 1995). Ann. Pure Appl. Logic 88 (1997), no. 2-3, 149-164. MR1600895 (99b:03049)

32. Komlós, J.; Simonovits, M., Szemerédi's regularity lemma and its applications in graph theory. Combinatorics, Paul Erdös is eighty, Vol. 2 (Keszthely, 1993), 295-352, Bolyai Soc. Math. Stud., 2, János Bolyai Math. Soc., Budapest, 1996. MR.1395865 (97d:05172)

33. Krauss, Peter H., Represetation of Symmetric Probability Models, J. Symbolic Logic 34, No. 2 (June 1969), pp. 183-193. MR.0275482 (43:1236)

34. Scott, D.; Krauss, P., Assigning probabilities to logical formulas, in: Aspects of inductive logic, ed. J. Hintikka and P. Suppes, North-Holland, Amsterdam, 1966, pp. 219-259. MR 0201269 $(34: 1153)$

35. Larsen, Michael J.; Pink, Richard, Finite subgroups of algebraic groups, 1998. Available from http://www.math.ethz.ch/ pink/publications.html.

36. Lee, John M., Riemannian manifolds: An introduction to curvature. Graduate Texts in Mathematics, 176. Springer-Verlag, New York, 1997. MR.1468735 (98d:53001)

37. Marker, David, Model theory. An introduction. Graduate Texts in Mathematics, 217. SpringerVerlag, New York, 2002. MR 1924282 (2003e:03060)

38. Marker, David, Semialgebraic Expansions of C, Transactions of the American Mathematical Society, Vol. 320, No. 2 (Aug., 1990), pp. 581-592. MR964900 (90k:03034)

39. Morley, Michael, The Löwenheim-Skolem theorem for models with standard part. 1971 Symposia Mathematica, Vol. V (INDAM, Rome, 1969/70), Academic Press, London, pp. 43-52. MR0284322 (44:1551)

40. Nikolov, Nikolay; Pyber, László, Product decompositions of quasirandom groups and a Jordan type theorem, arXiv:math/0703343.

41. Pillay, Anand, http://www.amsta.leeds.ac.uk/ pillay/.

42. Pillay, Anand, An introduction to stability theory. Oxford Logic Guides, 8. The Clarendon Press, Oxford University Press, New York, 1983. xii+146 pp. MR719195 (85i:03104)

43. Poizat, Bruno, Cours de théorie des modèles. Bruno Poizat, Lyon, 1985. vi+584 pp. MR817208 (87f:03084) 
44. Poizat, Bruno, An introduction to algebraically closed fields and varieties. in: The model theory of groups (Notre Dame, IN, 1985-1987), 41-67, Notre Dame Math. Lectures, 11, Univ. Notre Dame Press, Notre Dame, IN, 1989. MR.985339

45. Raghunathan, M. S., Discrete subgroups of Lie groups. Math. Student 2007, Special Centenary Volume, 59-70 (2008). MR2527560(2010d:22016)

46. Shalom, Yehuda; Tao, Terence, A finitary version of Gromov's polynomial growth theorem, arXiv:0910.4148v2 [math.GR].

47. Shelah, Saharon, The lazy model-theoretician's guide to stability. Comptes Rendus de la Semaine d'étude en Théorie des Modules (Inst. Math., Univ. Catholique Louvain, Louvain-laNeuve, 1975). Logique et Analyse (N.S.) 18 (1975), no. 71-72, 241-308. MR0539969(58:27447)

48. Shelah, S., Classification theory and the number of nonisomorphic models. Second edition. Studies in Logic and the Foundations of Mathematics, 92. North-Holland Publishing Co., Amsterdam, 1990. xxxiv+705 pp. MR.1083551 (91k:03085)

49. Shelah, Saharon, Simple unstable theories. Ann. Math. Logic 19 (1980), no. 3, 177-203. MR:595012(82g:03055)

50. Tao, Terence, Product set estimates for non-commutative groups, arXiv math/0601431, Combinatorica 28 (2008), 547-594. MR2501249 (2010b:11017)

51. Tao, Terence, The sum-product phenomenon in arbitrary rings, arXiv:0806.2497, Contrib. Discrete Math. 4 (2009), 59-82. MR2592424 (2011a:11020)

52. Tao, Terence, http://terrytao.wordpress.com/2007/03/02/open-question-noncommutativefreiman-theorem/.

53. Tao, Terence, Freiman's theorem for solvable groups, arXiv:0906.3535.

54. Tao, Terence; Vu, Van, Additive combinatorics. Cambridge Studies in Advanced Mathematics, 105. Cambridge University Press, Cambridge, 2006. xviii+512 pp. MR.2289012 (2008a:11002)

55. Yamabe, Hidehiko, A generalization of a theorem of Gleason. Ann. of Math. (2) 58 (1953), 351-365. MR0058607 (15:398d)

56. Weil, André, Foundations of algebraic geometry. American Mathematical Society, Providence, R.I., 1962, xx+363 pp. MR0144898 (26:2439)

57. Zimmer, Robert J., Essential results of functional analysis. Chicago Lectures in Mathematics. University of Chicago Press, Chicago, IL, 1990. x+157 pp. MR.1045444 (91h:46002)

Institute of Mathematics, Hebrew University at Jerusalem, Giv'at Ram, 91904 JeruSALEM, ISRAEL

E-mail address: ehud@math.huji.ac.il 